\title{
UNIFORM TREE LATTICES
}

\author{
HYMAN BASS AND RAVI KULKARNI
}

TABLE OF CONTENTS

0. Introduction

1. Graphs of groups of finite index; unimodularity Appendix A: Nonabelian cohomology of a graph

2. Finite groupings of edge indexed graphs; volumes

3. Automorphism groups of locally finite trees; unimodularity

4. Existence, conjugacy, and commensurability of uniform lattices

5. Volumes, Euler characteristics, and ranks

6. Finiteness properties

Appendix B: Commensurators

7. Nonfiniteness phenomena

\section{References}

\section{INTRODUCTION}

Let $X$ be a locally finite tree. Then $G=\operatorname{Aut}(X)$ is a locally compact group; the stabilizers $G_{x}$ are open and profinite. A subgroup $\Gamma \leq G$ is discrete if all $\Gamma_{x}$ are finite. We then call $\Gamma$ a lattice if

$$
\operatorname{Vol}(\Gamma \backslash \backslash X)=\sum_{x \in \Gamma \backslash X} \frac{1}{\left|\Gamma_{x}\right|}
$$

is finite, and a uniform lattice if the graph $\Gamma \backslash X$ is finite. These are the objects of our study.

It is fruitful to think of $(G, X, \Gamma)$ as a combinatorial analogue of $\left(\mathrm{PSL}_{2}(\mathbf{R})\right.$, upper half-plane, Fuchsian group). An even more direct analogy

Received by the editors February 28, 1990.

1980 Mathematics Subject Classification (1985 Revision). Primary 05C25, 20F32, 22 E40.

The authors were partially supported by NSF grants DMS 8802181 (first author) and DMS 8902 214 (second author). 
is with $\operatorname{PSL}_{2}(K), K$ a local nonarchimedean field, and its Bruhat-Tits tree. Our results show that, while these analogies are far reaching, they also break down in dramatic ways. For example, the Commensurability Theorem below implies that, if one uniform lattice is "arithmetic," then all are!

The first question is: When does $X$ admit a uniform lattice? The obvious necessary homogeneity condition, that $G \backslash X$ be finite, turns out to be not sufficient.

Existence Theorem. $X$ admits a uniform lattice iff $G \backslash X$ is finite and $G$ is unimodular (in the sense of Haar measure).

In this case we call $X$ a uniform tree. More generally, $H \leq G$ contains a uniform lattice iff $H \backslash X$ is finite and $\bar{H}$ (closure of $H$ ) is unimodular. This gives Lubotzky's results [Lub] on the existence of uniform lattices in rank one simple Lie groups over local fields.

A uniform lattice on $X$ contains a finite index subgroup acting freely on $X$. Hence, uniform trees are just the universal covers of finite connected graphs.

Let $X$ be a uniform tree. Put $\operatorname{Lat}_{u}(X)=$ the set of uniform lattices on $X$.

Commensurability Theorem. If $\Gamma_{0}, \Gamma_{1} \in \operatorname{Lat}_{u}(X)$, then $g \Gamma_{0} g^{-1}$ and $\Gamma_{1}$ are commensurable for some $g \in G$.

This is equivalent to the special case when $\Gamma_{0}$ and $\Gamma_{1}$ are free lattices, in which case the result can be reformulated, using covering theory, as

Leighton's Common Covering Theorem [L]. If two finite connected graphs have a common covering, then they have a common finite covering.

Indeed, it was the desire to give a covering space theoretic proof of Leighton's theorem that originally inspired this paper. The second named author produced a strategy for such a proof in $[\mathrm{K}]$. However, the theory of "branched coverings of graphs of groups" needed to execute that argument did not exist at the time. That theory has since been constructed in [B], and the present paper is an exploitation of that theory.

The Commensurability Theorem implies that the commensurator of $\Gamma \in$ $\operatorname{Lat}_{u}(X)$,

$$
C_{G}(\Gamma)=\left\{g \in G \mid g \Gamma g^{-1} \text { and } \Gamma \text { are commensurable }\right\},
$$

depends, up to conjugacy, only on $X$. We sometimes denote it $C(X)$, and call it the commensurability group of $X$. It is countable, and $C(X) \backslash X=G \backslash X$.

Conjectures. I. $C(X)$ is dense in $G$.

II. Let $\Gamma \leq G$ and let $Y$ be a $\Gamma$-invariant subtree of $X$ on which $\Gamma$ acts discretely with finite quotient $\Gamma \backslash Y$. Then there exist $\Gamma^{+} \in \operatorname{Lat}_{u}(X)$ and an injective homomorphism $h: \Gamma \rightarrow \Gamma^{+}$such that $h(g)|Y=g| Y$ for all $g \in \Gamma$.

We can show that II (for finite $\Gamma$ ) implies I, that II holds when $\Gamma$ is free, and that I and II hold when $X$ is a homogeneous tree or if $G \backslash X$ is a tree. 
Finiteness properties of lattices. Let $\Gamma \in \operatorname{Lat}_{u}(X)$. Put $m(\Gamma)=\operatorname{lcm}|F|$, where $F$ varies over finite subgroups $F$ of $\Gamma$. There are only finitely many conjugacy classes of such $F$, and $\Gamma$ contains a free subgroup of index $m(\Gamma)$. Moreover $N_{G}(\Gamma) / \Gamma$ is finite.

For $m_{0}>0$,

$$
U^{m_{0}}(\Gamma)=\left\{\Gamma^{\prime} \leq G \mid \Gamma \leq \Gamma^{\prime} \text { and }\left[\Gamma^{\prime}: \Gamma\right] \leq m_{0}\right\}
$$

is finite. For $v_{0}, m_{0}>0$,

$$
\operatorname{Lat}_{u}^{v_{0}, m_{0}}(X)=\left\{\Gamma \in \operatorname{Lat}_{u}(X) \mid \operatorname{Vol}(\Gamma \backslash \backslash X) \leq v_{0} \text { and } m(\Gamma) \leq m_{0}\right\}
$$

forms finitely many $G$-conjugacy classes. Put

$$
L(\Gamma, G)=\left\{h \in \operatorname{Hom}(\Gamma, G) \mid h \text { is injective and } h(\Gamma) \in \operatorname{Lat}_{u}(X)\right\} \text {. }
$$

If $X$ is not "virtually linear" (i.e., if $\Gamma$ is not virtually infinite cyclic), then

$$
G \backslash L(\Gamma, G) / \operatorname{Aut}(\Gamma)
$$

is finite, where $G$ acts by conjugation and $\operatorname{Aut}(\Gamma)$ by composition.

Nonfiniteness phenomena. Let $X_{n}$ denote the $n$-homogeneous tree; each vertex has index $n$. For $n \geq 3$, there exist infinite ascending chains of $\Gamma \in \operatorname{Lat}_{u}\left(X_{n}\right)$. In particular, $\operatorname{Vol}\left(\Gamma \backslash \backslash X_{n}\right) \rightarrow 0$ as $\Gamma$ increases.

For $n \geq 5$, given any integer $v>0, v$ even if $n=5$, there exist infinitely many conjugacy classes of $\Gamma \in \operatorname{Lat}_{u}\left(X_{n}\right)$ with $\operatorname{Vol}\left(\Gamma \backslash \backslash X_{n}\right)=v$.

Methods. Our method is based on interpreting tree actions as graphs of groups (cf. [S, (I.5)] or [B]). A graph of groups $\mathbf{A}=(A, \mathscr{A})$ consists of a connected graph $A$, vertex groups $\mathscr{A}_{a}(a \in A)$, edge groups $\mathscr{A}_{e}=\mathscr{A}_{\bar{e}}(e \in E(A))$, and monomorphisms $\alpha_{\varepsilon}: \mathscr{A}_{e} \rightarrow \mathscr{A}_{\partial_{0} e}$. We put $i(e)=\left[\mathscr{A}_{\partial_{0} e}: \alpha_{e} \mathscr{A}_{e}\right]$ and call $(A, i)=I(\mathbf{A})$ the corresponding edge-indexed graph. If $a_{0} \in A$, then the fundamental group $\Gamma=\pi_{1}\left(\mathbf{A}, a_{0}\right)$ acts without inversion (of edges) on the covering tree $X=\left(\widetilde{\mathbf{A}, a_{0}}\right)$, with quotient $p: X \rightarrow \Gamma \backslash X=A$ so that, for $e \in E(X), \alpha_{p(e)}: \mathscr{A}_{p(e)} \rightarrow \mathscr{A}_{p\left(\partial_{0} e\right)}$ is isomorphic to the inclusion $\Gamma_{e} \leq \Gamma_{\partial_{0} e}$. Conversely, every action without inversion of a group $\Gamma$ on a tree $X$ arises this way from a quotient graph of groups $\Gamma \backslash \backslash X=(\Gamma \backslash X, \mathscr{A})$.

Thus, constructing a tree action $(\Gamma, X)$ is equivalent to constructing a graph of groups $\mathbf{A}=(A, \mathscr{A})$. We do this in two steps. First construct $I(\mathbf{A})=(A, i)$. In fact $X=\left(\widetilde{\mathbf{A}, a_{0}}\right)$ depends only on $(A, i)$, so we also write $X=\left(A, \widetilde{i}, a_{0}\right)$. Given $(A, i)$, a graph of groups $\mathbf{A}$ with $I(\mathbf{A})=(A, i)$ is called a "grouping" of $(A, i)$; a "finite grouping" if all $\mathscr{A}_{a}(a \in A)$ are finite, and an "effective grouping" if $\Gamma=\pi_{1}\left(\mathbf{A}, a_{0}\right)$ acts effectively (i.e., faithfully) on $X$. Thus, to construct a uniform lattice on a tree $X$ we first seek a finite edge-indexed graph $(A, i)$ so that $X=\left(A \widetilde{i,} a_{0}\right)$ (for a base point $\left.a_{0} \in A\right)$, and we then seek a finite effective grouping $\mathbf{A}$ of $(A, i)$. Then $\Gamma=\pi_{1}\left(\mathbf{A}, a_{0}\right)$ is the desired uniform lattice, and $\operatorname{Vol}(\Gamma \backslash \backslash X)=\operatorname{Vol}(\mathbf{A})=\sum_{a \in A} 1 /\left|\mathscr{A}_{a}\right|$. This problem is an interesting mix of combinatorics and finite group theory. 
Let $(A, i)$ be a finite edge-indexed graph, $a_{0} \in A$. In $\S \S 1$ and 2 we show that $(A, i)$ admits a finite (effective) grouping iff $(A, i)$ is "unimodular" in the sense that, for any closed edge-path $\gamma=\left(e_{1}, \ldots, e_{n}\right)$ at $a_{0} \in A, \prod_{i=1}^{n} i\left(e_{i}\right)=$ $\prod_{i=1}^{n} i\left(\bar{e}_{i}\right)$. In any event, the formula $\Delta(\gamma)=\prod_{i=1}^{n} i\left(e_{i}\right) / i\left(\bar{e}_{i}\right)$ defines a homomorphism

$$
\Delta: \pi_{1}\left(A, a_{0}\right) \rightarrow \mathbf{Q}^{\times}
$$

which is trivial iff $(A, i)$ is unimodular.

Suppose that $(A, i)=I(H \backslash \backslash X)$, where $H$ is a group of automorphisms without inversion of a locally finite tree $X$. There is a natural projection $p: H=$ $\pi_{1}\left(H \backslash \backslash X, a_{0}\right) \rightarrow \pi_{1}\left(A, a_{0}\right)$, whence the composition

$$
\Delta^{H}=\Delta \circ p: H \rightarrow \mathbf{Q}^{\times} .
$$

Let $\bar{H}$ denote the closure of $H$ in $G=\operatorname{Aut}(X)$. In $\S 3$ we show that $\Delta^{H}=\Delta^{\bar{H}} \mid H$ and that $\Delta^{\bar{H}}$ is the modular homomorphism, in the sense of Haar measure. Thus, when $H \backslash X$ is finite, we have the following equivalent conditions:

(a) $\bar{H}$ is unimodular.

(b) $I(H \backslash \backslash X)$ is unimodular.

(c) $I(H \backslash \backslash X)$ admits a finite effective grouping.

(c) There is a $\Gamma \in \operatorname{Lat}_{u}(X)$ with $\Gamma \backslash X=H \backslash X$.

Under conditions (a) and (b), and hence all of the above conditions when $H \backslash X$ is finite, we say simply that " $H$ is unimodular."

The next task is to try to embed a $\Gamma$ as in (d) into $H$, via a conjugation in $G$. For this we need an appropriate notion of "covering morphism" $\Phi: \Gamma \backslash \backslash X \rightarrow$ $H \backslash \backslash X$ of graphs of groups, that will give rise to the desired embedding. It is here that we invoke the theory of such covering morphisms developed in [B]. Using that theory, it can be shown that, for a free subgroup $\Gamma^{\prime}$ of finite index in $\Gamma$, there is a covering morphism $\Gamma^{\prime} \backslash \backslash X \rightarrow H \backslash \backslash X$, whence a conjugation of $\Gamma^{\prime}$ into $H$. This is contained in the following Conjugacy Theorem.

Let $X$ be a tree, $G=\operatorname{Aut}(X), H \leq G$ without inversions, and put

$$
G_{H}=\{g \in G \mid g \text { stabilizes all } H \text {-orbits on } X\} \text {. }
$$

Conjugacy Theorem [B, (5.2)]. If $\Gamma \leq G_{H}$ acts freely on $X$, then $g \Gamma g^{-1} \leq H$ for some $g \in G_{H}$.

From this we deduce

Uniform Lattice Theorem. Assume that $X$ is locally finite, $H \backslash X$ is finite, and $H$ is unimodular.

(a) There is a lattice $\Phi \leq G_{H}$ such that $\Phi \backslash X=H \backslash X$.

(b) If $\Gamma \leq G_{H}$ acts freely on $X$, then $\Gamma$ can be $G_{H}$-conjugted into $H$ and into $\Phi$.

Using conjugation into $H$ gives the Existence Theorem. Using conjugation into $\Phi$ gives the Commensurability Theorem. These and many related results are obtained in $\S 4$. 
In $\S 5$ we discuss volumes, Euler characteristics, and other numerical invariants attached to uniform trees and their lattices. In $\S 6$ we establish the finiteness properties announced above. Following this is an appendix on commensurability groups of uniform tree lattices, and on their action on the ends of the corresponding trees.

The nonfiniteness phenomena are illustrated in $\S 7$ by a great variety of examples. The game here is to produce finite unimodular edge-indexed graphs $(A, i)$ with a prescribed covering tree $X$, and then either to find arbitrarily large finite effective groupings of $(A, i)$ or else to prove that there are only finitely many such. For example, consider

$$
(A, i)=\stackrel{m_{0} \quad m_{1}}{\circ} \text {. }
$$

Then $X$ is the bihomogeneous bipartite tree with indices $m_{0}$ and $m_{1}$. A finite effective grouping of $(A, i)$ is an "amalgam", $G_{0} \geq H \leq G_{1}$, where the $G_{i}$ are finite groups, $\left[G_{i}: H\right]=m_{i}(i=0,1)$, and if $N \leq H$ is normal in each $G_{i}$, then $N=1$. Assume that $m_{i} \geq 2(i=0,1)$. If $m_{0}$ or $m_{1}$ is composite, then there exist infinite ascending chains of finite effective groupings of $(A, i)$ (cf. (7.13)), and hence infinite ascending chains of edge-transitive lattices on $X$. On the other hand, if $m_{0}$ and $m_{1}$ are prime, then there are conjecturally only finitely many effective $\left(m_{0}, m_{1}\right)$-amalgams, i.e., only finitely many conjugacy classes of edge-transitive lattices on $X$. This follows for $m_{0}, m_{1} \leq 3$ from a well-known work of Goldschmidt [Go]. It has been proved more generally by Fan [F], whenever $H$ is a $p$-group for some prime $p \neq m_{0}$ or $m_{1}$.

\section{GRAPHS OF GROUPS OF FINITE INDEX; UNIMODULARITY}

Our background reference here is $[\mathrm{B}]$. (1.1). Let $\mathbf{A}=(A, \mathscr{A})$ be a graph of groups. For $e \in E(A), \partial_{0} e=a$, we put

$$
\mathscr{A}_{a / e}=\mathscr{A}_{a} / \alpha_{e}\left(\mathscr{A}_{e}\right)
$$

and

$$
i(e)\left(=i^{\mathbf{A}}(e)\right)=\left|\mathscr{A}_{a / e}\right|=\left[\mathscr{A}_{a}: \alpha_{e}\left(\mathscr{A}_{e}\right)\right] .
$$

We shall assume that " $\mathbf{A}$ has finite index," i.e., that all of these indices $i(e)$ are finite. We then call

$$
I(\mathbf{A})=(A, i)
$$

the edge-indexed graph of $\mathbf{A}$, and further define

$$
q(e)=i(e) / i(\bar{e}) \in \mathbf{Q}_{>0}^{\times}
$$

for $e \in E(A)$. Note that

$$
q(\bar{e})=q(e)^{-1} .
$$

Thus $q$ defines a homomorphism

$$
q: \pi(A) \rightarrow \mathbf{Q}_{>0}^{\times}
$$


where, viewing $A$ as a graph of trivial groups, $\pi(A)$ is defined as in [B, I, (1.5)] by the presentation

$$
\left.\pi(A)=\langle E(A)| \bar{e}=e^{-1} \text { for all } e \in E(A)\right\rangle .
$$

If $\gamma=\left(e_{1}, \ldots, e_{n}\right)$ is an edge path in $A$ we put $|\gamma|=e_{1} \cdots e_{n} \in \pi(A)$ and $q(\gamma)=q(|\gamma|)=q\left(e_{1}\right) \cdots q\left(e_{n}\right)$. Putting $\bar{\gamma}=\left(\bar{e}_{n}, \ldots, \bar{e}_{1}\right)$ we have $q(\bar{\gamma})=$ $q(\gamma)^{-1}$.

Choose a base point $a_{0} \in A$ and consider

$$
\pi_{1}\left(A, a_{0}\right)=\left\{|\gamma| \in \pi(A) \mid \gamma \text { is a closed path at } a_{0}\right\} \text {. }
$$

By restriction, $q$ defines a homomorphism

$$
\Delta_{a_{0}}(q): \pi_{1}\left(A, a_{0}\right) \rightarrow \mathbf{Q}_{>0}^{\times} .
$$

Composing with the natural surjection $\pi_{1}\left(\mathbf{A}, a_{0}\right) \stackrel{p}{\rightarrow} \pi_{1}\left(A, a_{0}\right)$ (cf. [B, (1.5), Example 3]) we obtain

$$
\Delta_{a_{0}}^{\mathbf{A}}=\Delta_{a_{0}}(q) \circ p: \pi_{1}\left(\mathbf{A}, a_{0}\right) \rightarrow \mathbf{Q}_{>0}^{\times} .
$$

(1.2) Proposition. The following conditions are equivalent.

(a) $\Delta_{a_{0}}^{\mathbf{A}}=1$.

(b) For any closed edge path $\gamma=\left(e_{1}, \ldots, e_{n}\right)$ in $A$,

$$
q(\gamma)\left(=q\left(e_{1}\right) \cdots q\left(e_{n}\right)\right)=1 \text {. }
$$

(c) For any edge path $\gamma=\left(e_{1}, \ldots, e_{n}\right)$ in $A, q(\gamma)$ depends only on the end points of $\gamma$.

(d) There is a function $N: A \rightarrow \mathbf{Q}_{>0}^{\times}$such that

$$
q(e)=N\left(\partial_{0} e\right) N\left(\partial_{1} e\right)^{-1} \text { for all } e \in E(A) .
$$

Under these conditions, the function $N$ in (d) is unique up to a constant factor.

Let $\gamma=\left(e_{1}, \ldots, e_{n}\right)$ be an edge path in $A$ with vertex sequence $b_{0}, b_{1}, \ldots$, $b_{n}$. Assuming (d) we have $q(\gamma)=N\left(b_{0}\right) N\left(b_{n}\right)^{-1}$, whence (c). Moreover, (c) $\Rightarrow$ (b) because a closed path at $a$ has the same end points as the empty edge path at $a$. Obviously, (b) $\Rightarrow(\mathrm{a})$.

Finally, to prove $(\mathrm{a}) \Rightarrow(\mathrm{d})$, define $N_{a_{0}}(a)=q(\gamma)^{-1}$, where $\gamma$ is any edge path from $a_{0}$ to $a$. Given $e \in E(A)$ let $\gamma_{i}$ be an edge path from $a_{0}$ to $\partial_{i} e$ $(i=0,1)$. Then $\gamma=\left(\gamma_{0}, e, \bar{\gamma}_{1}\right)$ is a closed path at $a_{0}$ so, by $(\mathrm{a})$,

$$
1=q(\gamma)=q\left(\gamma_{0}\right) q(e) q\left(\bar{\gamma}_{1}\right)=N_{a_{0}}\left(\partial_{0} e\right)^{-1} q(e) N_{a_{0}}\left(\partial_{1} e\right),
$$

so $q(e)=N_{a_{0}}\left(\partial_{0} e\right) N_{a_{0}}\left(\partial_{1} e\right)^{-1}$, whence $(\mathrm{d})$.

Suppose also that $q(e)=N^{\prime}\left(\partial_{0} e\right) N^{\prime}\left(\partial_{1} e\right)^{-1}$ for all $e$, for some $N^{\prime}: A \rightarrow$ $\mathbf{Q}_{>0}^{\times}$. Put $M(a)=N_{a_{0}}(a)^{-1} N^{\prime}(a)$. The hypothesis

$$
N_{a_{0}}\left(\partial_{0} e\right) N_{a_{0}}\left(\partial_{1} e\right)^{-1}=N^{\prime}\left(\partial_{0} e\right) N^{\prime}\left(\partial_{1} e\right)^{-1}
$$


implies that $M\left(\partial_{0} e\right)=M\left(\partial_{1} e\right)$ for all $e \in E(A)$. Since $A$ is connected, $M$ is constant.

A more general (nonabelian) version of (1.2) is presented in Appendix A, Proposition (A.2).

(1.3) Definitions. Under the conditions of (1.2) we call $\mathbf{A}$ and $(A, i)$ unimodular. The functions $N: A \rightarrow \mathbf{Q}_{>0}^{\times}$such that $q(e)=N\left(\partial_{0} e\right) / N\left(\partial_{1} e\right)$ as in (1.2)(d) are called vertex orderings of $(A, i)$. We call $N$ integral if, for all $e \in E(A)$, $N\left(\partial_{0} e\right) / i(e) \in \mathbf{Z}$ and (hence) $N(a) \in \mathbf{Z}$ for $a \in A$. We further define

$$
\operatorname{Vol}_{N}(A, i)=\sum_{a \in A} 1 / N(a) \quad(\leq \infty)
$$

and say that $(A, i)$ has finite volume, denoted $\operatorname{Vol}(A, i)<\infty$, if $\operatorname{Vol}_{N}(A, i)<$ $\infty$ for some $N$. If $N^{\prime}$ is another vertex ordering, then $N^{\prime}=w N$ for some $w \in Q_{>0}^{\times}$, and so $\operatorname{Vol}_{N}(A, i)=w \operatorname{Vol}_{N^{\prime}}(A, i)$. Hence, finiteness of $\operatorname{Vol}(A, i)$ does not depend on the choice of $N$.

Let $(A, i)$ be unimodular with vertex ordering $N$. For $e \in E(A)$ put $r(e)=N\left(\partial_{0} e\right) / i(e)$. We say that $(A, i)$ has bounded denominators if the rational numbers $r(e) \quad(e \in E(A))$ have bounded denominators. As above, this condition does not depend on the choice of $N$.

(1.4) Proposition. The following conditions on $(A, i)$ are equivalent.

(a) $(A, i)$ admits an integral vertex ordering $N: A \rightarrow \mathbf{Z}_{>0}$.

(b) $(A, i)$ is unimodular and has bounded denominators.

Under these conditions there is a unique integral vertex ordering $N_{\min }$ such that every other one is of the form $N=m \cdot N_{\min }$ for some integer $m>0$.

Proof. If $N$ is an integral vertex ordering of $(A, i)$, then $(A, i)$ is unimodular, by (1.2), and the rational numbers $r(e)=N\left(\partial_{0} e\right) / i(e)$ are integers, hence have bounded denominators. Conversely, assuming (b), $(A, i)$ admits a vertex ordering $N$ so that the numbers $r(e)=N\left(\partial_{0} e\right) / i(e)$ have bounded denominators. Adjusting by a scalar factor we may assume that $N\left(a_{0}\right)=1$ for some $a_{0} \in A$. This done, let $D$ be the least common denominator of the numbers $r(e)$, and put $N_{\min }=D \cdot N$. Then, by construction, $N_{\min }$ is an integral vertex ordering. If $N^{\prime}$ is any integral vertex ordering we can write $N^{\prime}=q \cdot N$ for some $q \in Q$. Then $N^{\prime}\left(a_{0}\right)=q N\left(a_{0}\right)=q \in \mathbf{Z}$. Moreover, for all $e \in E(A)$, $N^{\prime}\left(\partial_{0} e\right) / i(e)=q N\left(\partial_{0} e\right) / i(e)=q r(e) \in \mathbf{Z}$. It follows that $q$ is a common denominator for the $r(e)$ 's; hence $q=m D$ and $N^{\prime}=m N_{\text {min }}$ for some integer $m>0$. This proves the proposition.

(1.5) Examples. 1. Suppose that

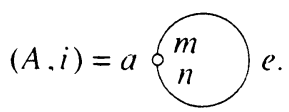

This is unimodular iff $n=m$, in which case $q(e)=1$, and $N_{\min }(a)=n$. 
2. Suppose

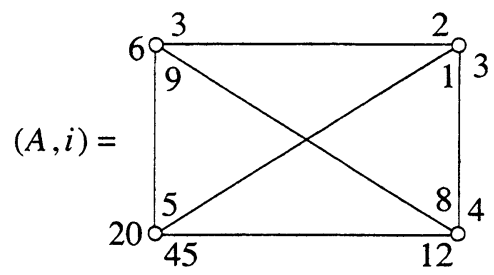

is unimodular. The minimal integral vertex ordering is

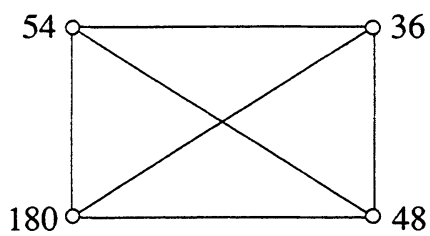

3. For $(A, i)$ unimodular the bounded denominator condition clearly holds if the graph $A$ is finite, but not in general. For example, if

$$
A=\left(\stackrel{e_{1}}{\longrightarrow} \stackrel{e_{2}}{\longrightarrow} \cdots \cdots \stackrel{e_{n}}{\longrightarrow} \cdots \cdots\right),
$$

then for the indexing $i\left(e_{n}\right)=1, i\left(\bar{e}_{n}\right)=2$ for all $n,(A, i)$ has bounded denominators, whereas, for the indexing $j\left(e_{n}\right)=2, j\left(\bar{e}_{n}\right)=1$ for all $n,(A, j)$ does not. Note that all edge-indexings are unimodular since $A$ is a tree.

\section{APPENDIX A: NONABELIAN COHOMOLOGY OF A GRAPH}

The material here is not needed elsewhere in the paper.

(A.1). Let $Q$ be a (multiplicative) group. For any set $A$ let $Q^{A}$ denote the group of set functions $A \rightarrow Q$.

Suppose that $A$ is a graph, with edge set $E(A)$ and end point maps $\partial_{i}: E(A)$ $\rightarrow A(i=0,1)$. Then we have a map

$$
\delta: Q^{A} \rightarrow\left(Q^{E(A)}\right)^{-}
$$

defined, for $N \in Q^{A}$, by

$$
(\delta N)(e)=N\left(\partial_{0} e\right) N\left(\partial_{1} e\right)^{-1}
$$

and

$$
\left(Q^{E(A)}\right)^{-}=\left\{q \in Q^{E(A)} \mid q(\bar{e})=q(e)^{-1} \text { for } e \in E(A)\right\}
$$

Recall that

$$
\left.\pi(A)=\langle E(A)| \bar{e}=e^{-1} \text { for } e \in E(A)\right\rangle,
$$

so that we can identify

$$
\left(Q^{E(a)}\right)^{-}=\operatorname{Hom}(\pi(A), Q) .
$$

Choose $a_{0} \in A$. Then $\pi_{1}\left(A, a_{0}\right)$ is the group of all elements $e_{1} \cdots e_{n} \in \pi(A)$ such that $\left(e_{1}, \ldots, e_{n}\right)$ is a closed edge path in $A$ at $a_{0}$. For $g \in\left(Q^{E(A)}\right)^{-}$we put

$$
\Delta_{a_{0}}(q)=q \mid \pi_{1}\left(A, a_{0}\right): \pi_{1}\left(A, a_{0}\right) \rightarrow Q .
$$


Finally, let $\gamma: Q \rightarrow Q^{A}$ be the diagonal embedding sending $s \in Q$ to the constant function $\gamma(s): a \mapsto s \quad(a \in A)$. These maps assemble into the sequence of pointed sets

$$
Q \stackrel{\vartheta}{\rightarrow} Q^{A} \stackrel{\delta}{\rightarrow}\left(Q^{E(A)}\right)^{-} \stackrel{\bar{\Delta}}{\rightarrow} Q \backslash \operatorname{Hom}\left(\pi_{1}\left(A, a_{0}\right), Q\right),
$$

where the quotient on the right is by the conjugation action of $Q$ and $\bar{\Delta}$ is induced by $\Delta_{a_{0}}$. We shall show that the sequence (1) is "exact" in a strong sense.

(A.2) Proposition. Suppose that $A$ is a connected graph.

(a) $\gamma$ is an injective group homomorphism.

(b) The fibers of $\delta$ are the $\gamma(Q)$-right cosets; $\delta N^{\prime}=\delta N$ iff for some $s \in Q$, $N^{\prime}(a)=N(a) s$ for all $a \in A$.

(c) The group $Q^{A}$ acts on the set $\left(Q^{E(A)}\right)^{-}$by

$$
(N \cdot q)(e)=N\left(\partial_{0} e\right) q(e) N\left(\partial_{1} e\right)^{-1}
$$

for $N \in Q^{A}, q \in\left(Q^{E(A)}\right)^{-}$, and $e \in E(A) ; \delta$ is the corresponding orbit map at the constant function $q_{1}: q_{1}(e)=1$ for all $e \in E(A)$. We have

$$
\Delta_{a_{0}}(N \cdot q)=\operatorname{ad}\left(N\left(a_{0}\right)\right) \circ \Delta_{a_{0}}(q) .
$$

Moreover, the stabilizer $\left(Q^{A}\right)_{q}$ of $q \in\left(Q^{E(A)}\right)^{-}$admits the isomorphism $\left(Q^{A}\right)_{q} \rightarrow$ $Z_{Q}\left(\operatorname{Im}\left(\Delta_{a_{0}}(q)\right)\right)$ sending $N$ to $N\left(a_{0}\right)$.

(d) The fibers $\bar{\Delta}$ are the $Q^{A}$-orbits.

Proof. (a) Obvious.

(b) Evidently, $\delta(N \gamma(s))=\delta(N)$ for $N \in Q^{A}$ and $s \in Q$. Suppose that $N^{\prime} \in Q^{A}$ and $\delta N^{\prime}=\delta N$. Let $s=N^{\prime}\left(a_{0}\right)^{-1} N\left(a_{0}\right)$. Replacing $N^{\prime}$ by $N^{\prime} \gamma(s)$, we may assume that $N^{\prime}\left(a_{0}\right)=N\left(a_{0}\right)$. If $e \in E(A)$ and $N^{\prime}\left(\partial_{0} e\right)=N\left(\partial_{0} e\right)$, then, since $\delta N^{\prime}=\delta N$, we have $N^{\prime}\left(\partial_{1} e\right)=N\left(\partial_{1} e\right)$. Thus, $N^{\prime}=N$ since $A$ is connected.

(c) Let $a \in A$ and let $\alpha=\left(e_{1}, \ldots, e_{n}\right)$ be an edge path in $A$ from $a_{0}$ to $a$, with vertex sequence $a_{0}, a_{1}, \ldots, a_{n}=a$. Let $q \in\left(Q^{E(A)}\right)^{-}=\operatorname{Hom}(\pi(A), Q)$ and $N \in Q^{A}$. Then $N \cdot q: \pi(A) \rightarrow Q$ sends $|\alpha|=e_{1} \cdots e_{n} \in \pi(A)$ to

$$
\begin{aligned}
(N \cdot q)(|\alpha|)= & \left(N\left(a_{0}\right) q\left(e_{1}\right) N\left(a_{1}\right)^{-1}\right)\left(N\left(a_{1}\right) q\left(e_{2}\right) N\left(a_{2}\right)^{-1}\right) \\
& \cdots\left(N\left(a_{n-1}\right) q\left(e_{n}\right) N\left(a_{n}\right)^{-1}\right) \\
= & N\left(a_{0}\right)\left(q\left(e_{1}\right) q\left(e_{2}\right) \cdots q\left(e_{n}\right)\right) N\left(a_{n}\right)^{-1} \\
= & N\left(a_{0}\right) q(|\alpha|) N\left(a^{-1},\right.
\end{aligned}
$$

whence formula (2), when $a=a_{0}$. We further see that

$$
N \cdot q=q \text { iff }\left\{\begin{array}{l}
N(a)=q(|\alpha|)^{-1} N\left(a_{0}\right) q(|\alpha|) \\
\text { whenever } \alpha \text { is an edge path from } a_{0} \text { to } a .
\end{array}\right.
$$


Taking $a=a_{0}$, this condition implies that $N\left(a_{0}\right)$ centralizes $q\left(\pi_{1}\left(A, a_{0}\right)\right)=$ $\operatorname{Im}\left(\Delta_{a_{0}}(q)\right)$. Further, $N\left(a_{0}\right)$ (and $q$ ) determine $N$, whence a monomorphism $N \mapsto N\left(a_{0}\right)$ from $\left(Q^{A}\right)_{q}$ to $Z_{Q}\left(\operatorname{Im}\left(\Delta_{a_{0}}(q)\right)\right)$. To show surjectivity, let $s \in Q$ centralize $q\left(\pi_{1}\left(A, a_{0}\right)\right)$. If $\alpha$ is an edge path from $a_{0}$ to $a$ in $A$, then $N_{s}(a):=q(|\alpha|)^{-1} s q(|\alpha|)$ is well defined, since two such paths differ by composition with a closed path at $a_{0}$. By construction, $N_{s} \in\left(Q^{A}\right)_{q}$, and $N_{s}\left(a_{0}\right)=s$.

(d) Formula (2) shows that $\bar{\Delta}$ is constant on $Q^{A}$-orbits. Suppose that $q, q^{\prime} \in$ $\left(Q^{E(A)}\right)^{-}$and

$$
\Delta_{a_{0}}\left(q^{\prime}\right)=\operatorname{ad}(h) \circ \Delta_{a_{0}}(q)
$$

for some $h \in Q$. For $a \in A$ define

$$
N(a)=q^{\prime}(|\alpha|)^{-1} h q(|\alpha|),
$$

where $\alpha$ is an edge path from $a_{0}$ to $a$. Any other such path is equivalent to one of the form $\alpha^{\prime}=(\beta, \alpha)$, where $\beta$ is a closed path at $\alpha_{0}$, and we have $q^{\prime}(|\beta|)=h q(|\beta|) h^{-1}$ by (3). Hence

$$
\begin{aligned}
q^{\prime}\left(\left|\alpha^{\prime}\right|\right)^{-1} h q\left(\left|\alpha^{\prime}\right|\right) & =q^{\prime}(|\alpha|)^{-1} q^{\prime}(|\beta|)^{-1} h q(|\beta|) q(|\alpha|) \\
& =q^{\prime}(|\alpha|)^{-1} h q(|\alpha|),
\end{aligned}
$$

so $N$ is well defined. We conclude the proof by showing that $N \cdot q=q^{\prime}$. Let $e \in E(A), \partial_{0} e=a, \partial_{1} e=b$. Let $\alpha$ be an edge path from $a_{0}$ to $a$, and $\beta$ an edge path from $a_{0}$ to $b$. Then $(\alpha, e, \bar{\beta})$ is a closed path at $a_{0}$. From (3),

$$
q^{\prime}(|\alpha, e, \bar{\beta}|)=q^{\prime}(|\alpha|) q^{\prime}(e) q^{\prime}(|\beta|)^{-1}
$$

coincides with

$$
\begin{aligned}
h q(|\alpha, e, \bar{\beta}|) h^{-1} & =h q(|\alpha|) q(e) q(|\beta|)^{-1} h^{-1} \\
& =q^{\prime}(|\alpha|) N(a) q(e) N(b)^{-1} q^{\prime}(|\beta|)^{-1},
\end{aligned}
$$

by (4). Hence, $q^{\prime}(e)=N(a) q(e) N(b)^{-1}=(N \cdot q)(e)$, as was to be shown.

\section{FINITE GROUPINGS OF EDGE-INDEXED GRAPHS; VOLUMES}

(2.1) Consider an edge-indexed graph $(A, i)$. Thus, $A$ is a connected graph and $i$ assigns to each edge $e \in E(A)$ an integer $i(e)>0$.

For example, a graph of groups $\mathbf{A}=(A, \mathscr{A})$ of finite index gives rise to the edge-indexed graph $I(\mathbf{A})=\left(A, i^{\mathbf{A}}\right)$, where $i^{\mathbf{A}}(e)=\left[\mathscr{A}_{\partial_{0} e}: \alpha_{e}\left(\mathscr{A}_{e}\right)\right]$, which is finite by hypothesis. If $i^{\mathbf{A}}=i$, then we call $\mathbf{A}$ a grouping of $(A, i)$; a finite grouping if the groups $\mathscr{A}_{a}$ are all finite, and an effective grouping if $\mathbf{A}$ is an effective graph of groups (cf. [B, I, (1.24)]), i.e., if, for $a \in A, \pi_{1}(\mathbf{A}, a)$ acts effectively on $(\widetilde{\mathbf{A}, a})$. In general, if $\mathbf{A}^{\prime}=\left(A, \mathscr{A}^{\prime}\right)$ is the effective quotient of $\mathbf{A}$, in the sense of $[\mathrm{B}, \mathrm{I},(1.25)]$, then $i^{\mathbf{A}^{\prime}}=i^{\mathbf{A}}$. 
When $\mathbf{A}$ is a graph of finite groups we define its volume to be

$$
\operatorname{Vol}(\mathbf{A})=\sum_{a \in A} 1 /\left|\mathscr{A}_{a}\right|
$$

(which is possibly infinite).

We present criteria for the existence of finite groupings of $(A, i)$. (2.2) $(A, i)$ admits the following canonical infinite cyclic grouping $\mathbf{Z}(A, i)=$ $(A, \mathscr{A})$, where $\mathscr{A}_{a}=\mathscr{A}_{e}=\mathbf{Z}$ for all $a \in A, e \in E(A)$, and where $\alpha_{e}: \mathscr{A}_{e} \rightarrow$ $\mathscr{A}_{\partial_{0} e}$ is multiplication by $i(e)$. In case this is not effective the effective quotient $\mathbf{Z}(A, i)^{\prime}=\left(A, \mathscr{A}^{\prime}\right)$ is an effective finite cyclic grouping of $(A, i)$.

(2.3) Proposition. 1. If $\mathbf{A}=(A, \mathscr{A})$ is a finite grouping of $(A, i)$, then $N(a)=$ $\left|\mathscr{A}_{a}\right|(a \in A)$ defines an integral vertex ordering of $(A, i)$, in the sense of (1.2), i.e.,

$$
N\left(\partial_{0} e\right) / N\left(\partial_{1} e\right)=i(e) / i(\bar{e})
$$

for all $e \in E(A)$.

2. Let $N: A \rightarrow \mathbf{Z}$ be an integral vertex ordering of $(A, i)$. For $e \in E(A)$ put $N(e)=N\left(\partial_{0} e\right) / i(e) \quad(\in \mathbf{Z})$. For an integer $m>0$ let $\mu_{m}$ denote the complex mth roots of unity. Then $(A, i)$ admits the finite (cyclic) grouping $\mathbf{A}=(A, \mathscr{A})$, where $\mathscr{A}_{a}=\mu_{N(a)}, \mathscr{A}_{e}=\mu_{N(e)}$, and $\alpha_{e}: \mathscr{A}_{e} \rightarrow \mathscr{A}_{\partial_{0} e}$ is the inclusion for all $a \in A, e \in E(A)$.

Proof. (1) Let $\partial_{0} e=a, \partial_{1} e=b$. Then

$$
\frac{N(a)}{N(b)}=\frac{\left|\mathscr{A}_{a}\right|}{\left|\mathscr{A}_{b}\right|}=\frac{\left|\mathscr{A}_{a}\right|}{\left|\mathscr{A}_{e}\right|} \cdot \frac{\left|\mathscr{A}_{\bar{e}}\right|}{\left|\mathscr{A}_{b}\right|}=\frac{i(e)}{i(\bar{e})} .
$$

Assertion (2) is clear.

(2.4) Corollary. The following conditions on $(A, i)$ are equivalent.

(a) $(A, i)$ admits an integral vertex ordering.

(b) $(A, i)$ is unimodular and has bounded denominators.

(c) $(A, i)$ admits a finite grouping.

Under these conditions, $(\mathbf{A}, i)$ admits an effective finite cyclic grouping $\mathbf{A}^{0}=$ $\left(A, \mathscr{A}^{0}\right)$ such that, for any finite grouping $\mathbf{A}=(A, \mathscr{A})$ of $(A, i)$, there is an integer $m=m\left(\mathbf{A} / \mathbf{A}^{0}\right)>0$ such that

$$
\left|\mathscr{A}_{a}\right|=m\left|\mathscr{A}_{a}^{0}\right| \text { for all } a \in A .
$$

Consequently,

$$
\operatorname{Vol}(\mathbf{A})=\frac{1}{m} \operatorname{Vol}\left(\mathbf{A}^{0}\right)
$$

Proof. We have (a) $\Leftrightarrow$ (b) from (1.4) and (b) $\Leftrightarrow$ (c) from (2.3). Let $N_{\min }$ be the minimal integral vertex ordering (cf. (1.4)), and let $\mathbf{A}^{0}$ be the cyclic grouping of (2.3)(2) with $\left|\mathscr{A}_{a}^{0}\right|=N_{\text {min }}(a)$. If $\mathbf{A}=(A, \mathscr{A})$ is another finite grouping, then $N(a)=\left|\mathscr{A}_{a}\right|$ is an integral vertex ordering $((2.3)(1))$ so $N=m \cdot N_{\min }$ for some integer $m>0$, by (1.4). It remains to see that $\mathbf{A}^{0}$ is effective. 
Let $\mathbf{A}^{\prime}$ denote the effective quotient of $\mathbf{A}^{0}[\mathrm{~B},(1.25)]$ and put $N^{\prime}(a)=\left|\mathscr{A}_{a}^{\prime}\right|$. Then $N^{\prime}=m \cdot N_{\min }$ for some integer $m>0$. But $N^{\prime}(a)=\left|\mathscr{A}_{a}^{\prime}\right|$ divides $N_{\text {min }}(a)=\left|\mathscr{A}_{a}^{0}\right|$ since $\mathscr{A}_{a}^{\prime}$ is a quotient group of $\mathscr{A}_{a}^{0}$. Hence, $m=1$ so $\mathbf{A}^{\prime}=\mathbf{A}^{0}$. The corollary now follows.

(2.5) Corollary. If the graph $A$ is finite, then $(A, i)$ admits a finite grouping iff $(A, i)$ is unimodular.

In fact the bounded denominator condition is automatic when $A$ is finite.

(2.6) Remark. If $\mathbf{A}$ is a finite grouping of $(A, i)$, then it follows from (2.4) that the finiteness of $\operatorname{Vol}(\mathbf{A})$ depends only on $(A, i)$, not on $\mathbf{A}$. Moreover, there is a maximum possible value of $\operatorname{Vol}(\mathbf{A})$, given by

$$
\operatorname{Vol}_{\text {max }}(A, i)=\sum_{a \in A} \frac{1}{N_{\text {min }}(a)} .
$$

We shall later investigate the existence of positive lower bounds for Vol(A) (see $\S 7)$.

The next result is recalled from [B, I, (3.10)]; see also [S, (II, 2.6)].

(2.7) Proposition. Let $\mathbf{A}=(A, \mathscr{A})$ be a graph of finite groups, $a_{0} \in A$, and $\Gamma=\pi_{1}\left(\mathbf{A}, a_{0}\right)$. Then $\Gamma$ has a free subgroup of finite index iff the orders $\left|\mathscr{A}_{a}\right|$ $(a \in A)$ are bounded. If the latter are bounded put $m=m(\mathbf{A})=\operatorname{lcm}_{a \in A}\left|\mathscr{A}_{a}\right|$. Then there is a free subgroup $\Gamma^{0}$ of index $m$ in $\Gamma$. Any other free subgroup has index a multiple of $m$.

(2.8) Corollary. Let $\Gamma$ be a group acting without inversion on a tree $X$ with finite stabilizers $\Gamma_{x}$. Then $\Gamma$ is virtually free iff the orders $\left|\Gamma_{x}\right|(x \in \Gamma \backslash X)$ are bounded. In this case put $m=\operatorname{lcm}_{x \in \Gamma \backslash X}\left|\Gamma_{x}\right|$. Then $\Gamma$ contains a free subgroup of index $m$, and any other free subgroup has index a multiple of $m$.

Proof. Apply (2.7) to $\mathbf{A}=\Gamma \backslash \backslash X$, and use [B, I, (3.7)].

\section{AUTOMORPHISM GROUPS OF LOCALLY FINITE TREES; UNIMODULARITY}

(3.1) We fix the following notation in this section:

$$
\begin{aligned}
& X=\text { a tree. } \\
& G=\operatorname{Aut}(X) . \\
& d=\text { the (edge path) distance function on } X .
\end{aligned}
$$

We recall from $[B,(6.3)]$ that there is an exact sequence

$$
1 \rightarrow G^{0} \rightarrow G \stackrel{\bar{l}}{\rightarrow} \mathbf{Z} / 2 \mathbf{Z},
$$

where $\bar{l}(s)=[d(s x, x) \bmod 2]$ for all $x \in X$, and $G^{0}$ acts without inversion on $X$.

(3.2) For $x \in X$ we have

$$
B_{x}(r)=\{y \in X \mid d(x, y) \leq r\},
$$


the ball of radius $r(\geq 0)$ centered at $x$. The stabilizer $G_{x}$ leaves $B_{x}(r)$ invariant and it is easily seen that

$$
G_{x}=\underset{r \rightarrow \infty}{\lim } \operatorname{Im}\left(G_{x} \rightarrow \operatorname{Aut}\left(B_{x}(r)\right)\right)
$$

(3.3) Assume henceforth that $X$ is locally finite, i.e., that

$$
\operatorname{St}(x)=\left\{e \in E(X) \mid \partial_{0} e=x\right\}
$$

is finite for all $x \in X$. It follows then that $B_{x}(r)$ is finite for all $r \geq 0$, and so, the inverse limit (2) shows that

$$
G_{x} \text { is a profinite group. }
$$

We topologize $G$ so that two automorphisms are close if they agree on a large finite subtree of $X$. Then the $G_{x}$ 's are compact open subgroups, and $G$ is locally compact. Note that $G^{0}$ in (1) is a closed subgroup. In fact, $l: G \rightarrow \mathbf{Z}$ is continuous.

(3.4) Let $H \leq G$ be a closed, hence locally compact, subgroup of $G$. (Being closed is equivalent to $H_{x}$ being closed in $G_{x}$ for all $x$.) Let

$$
\Delta^{H}: H \rightarrow \mathbf{R}_{>0}^{\times}
$$

be the modular homomorphism of $H$ (cf. [D, (XIV, 3)]). Recall that this is defined as follows. Let $\mu$ be a left-invariant Haar measure on $H$. For $h \in$ $H$ and $S$ a measurable subset of $H, \mu(h S)=\mu(S)$. Moreover, $\mu_{h}(S):=$ $\mu(S h)$ defines another left-invariant Haar measure, so $\mu_{h}=\Delta^{H}(h) \cdot \mu$ for some $\Delta^{H}(h)>0$; this defines $\Delta^{H}$. If $H^{0} \leq H$ is an open subgroup, then $\Delta^{H} \mid H^{0}=$ $\Delta^{H^{0}}$.

Since compact open subgroups of $H$ are commensurable, we can scale $\mu$ so as to take rational values on all of them.

(3.5) Let $H \leq G$ be a subgroup that acts without inversions on $X$. Then (cf. $[\mathrm{B},(3.2)])$ we can form a quotient graph of groups

$$
H \backslash \backslash X=(H \backslash X, \mathscr{H})
$$

and the edge-indexed graph

$$
I(H \backslash \backslash X)=\left(H \backslash X, i^{H}\right),
$$

where $i^{H}(e)=\left[\mathscr{H}_{\partial_{0} e}: \alpha_{e} \mathscr{H}_{e}\right]$ is finite because $X$ is locally finite. As in (1.1), we obtain, relative to a base point $a_{0} \in H \backslash X$, a homomorphism

$$
\Delta^{H \backslash \backslash X}: H=\pi_{1}\left(H \backslash \backslash X, a_{0}\right) \rightarrow \mathbf{Q}_{>0}^{\times},
$$

where we use $[\mathrm{B}, \mathrm{I},(3.7)]$ to identify $H$ with $\pi_{1}\left(H \backslash \backslash X, a_{0}\right)$. Since $\mathbf{Q}_{>0}^{\times}$is abelian, $\Delta^{H \backslash \backslash X}$ does not depend on $a_{0}$ (cf. [B, (1.22)]).

Let $\bar{H}$ denote the closure of $H$ in $G$. Then it is easily seen that $\bar{H}$ also acts without inversions on $X$, that $\bar{H} \backslash X=H \backslash X$, and that we can construct $\bar{H} \backslash \backslash X$ 
so that $H \backslash \backslash X$ is a full graph of subgroups of $\bar{H} \backslash \backslash X$ [B, I, (3.9)]. Moreover, $I(\bar{H} \backslash \backslash X)=I(H \backslash \backslash X)$ and $\Delta_{a_{0}}^{\bar{H} \backslash X X} \mid H=\Delta_{a_{0}}^{H \backslash X}$. The homomorphism $\Delta_{a_{0}}^{\bar{H} \backslash X X}$ is trivial on the (open) subgroup generated by all vertex stabilizers so it is continuous, hence determined by $\Delta_{a_{0}}^{H \backslash \backslash X}$ on the dense subgroup $H$.

(3.6) Proposition. Let $H \leq G$ be a closed subgroup acting without inversion on $X$. Then the modular homomorphism $\Delta^{H}: H \rightarrow \mathbf{R}_{>0}^{\times}$agrees with $\Delta_{a_{0}}^{H \backslash X}: H=$ $\pi_{1}\left(H \backslash \backslash X, a_{0}\right) \rightarrow \mathbf{Q}_{>0}^{\times}$.

Proof. Say $H \backslash \backslash X=(H \backslash X, \mathscr{H})$ is formed relative to : btrees $X \supset S \supset T$ and $\left(h_{x}\right)_{x \in S}$, as in [B, (3.2)]. Let $e \in E(H \backslash X), \partial_{0} e=a, \partial_{1} e=b$. Then

$$
\mathscr{H}_{a} \stackrel{\alpha_{e}}{\leftarrow} \mathscr{H}_{e} \stackrel{\alpha_{\bar{q}}}{\rightarrow} \mathscr{H}_{b}
$$

coincides with

$$
H_{a^{T}} \stackrel{\operatorname{ad}\left(h_{0}\right)}{\leftarrow} H_{e^{s}} \stackrel{\operatorname{ad}\left(h_{1}\right)}{\rightarrow} H_{b^{T}},
$$

where $a^{T} \in T$ covers $a, e^{S} \in E(S)$ covers $e$, and $h_{0}=h_{\partial_{0} e^{s}}$, so $h_{0}\left(\partial_{0} e^{S}\right)=$ $a^{T}$; similarly for $b^{T}, h_{1}$. The groups $H_{a^{T}}, H_{e^{s}}, H_{b^{T}}$ are compact open and $h_{0} H_{e^{s}} h_{0}^{-1} \leq H_{a^{T}}$ with index $i(e)$, where $i=i^{H}$. Hence, if $\mu$ is a left-invariant Haar measure on $H$, we have

$$
\begin{aligned}
\mu\left(\mathscr{C}_{a}\right) & =\mu\left(H_{a^{r}}\right)=i(e) \cdot \mu\left(h_{0} H_{e} s h_{0}^{-1}\right) \\
& =i(e) \Delta^{H}\left(h_{0}\right)^{-1} \mu\left(H_{e^{s}}\right)=i(e) \Delta^{H}\left(h_{0}\right)^{-1} \mu\left(\mathscr{H}_{e}\right) .
\end{aligned}
$$

Similarly,

$$
\begin{aligned}
\mu\left(\mathscr{H}_{b}\right) & =i(\bar{e}) \Delta^{H}\left(h_{1}\right)^{-1} \cdot \mu\left(\mathscr{H}_{e}\right) \\
& =\frac{i(\bar{e}) \Delta^{H}\left(h_{0}\right)}{i(e) \Delta^{H}\left(h_{1}\right)} \cdot \mu\left(\mathscr{H}_{a}\right)=\left(\Delta^{H}\left(h_{e}\right) / q(e)\right) \cdot \mu\left(\mathscr{H}_{a}\right),
\end{aligned}
$$

where $h_{e}=h_{0} h_{1}^{-1} \in H$ and $q(e)=i(e) / i(\bar{e})$.

Let $\gamma=\left(e_{1}, \ldots, e_{n}\right)$ be an edge path with vertex sequence $a_{0}, a_{1}, \ldots, a_{n}$. Then $|\gamma|=e_{1} \cdots e_{n} \in \pi(H \backslash \backslash X)$, and $q(\gamma)=q(|\gamma|)=q\left(e_{1}\right) q\left(e_{2}\right) \cdots q\left(e_{n}\right)$. We also put $h_{\gamma}=h_{e_{1}} h_{e_{2}} \cdots h_{e_{n}}=h_{a_{0}} h_{a_{n}}^{-1} \in H$. It follows from (8) above that

$$
\mu\left(\mathscr{H}_{a_{n}}\right)=\Delta^{H}\left(h_{\gamma}\right) / q(\gamma) \cdot \mu\left(\mathscr{H}_{a_{0}}\right) .
$$

Taking for $\gamma$ a closed path at $a_{0}$ we conclude that

$$
\Delta^{H}\left(h_{\gamma}\right)=q(\gamma)
$$

for all closed paths $\gamma$ at $a_{0}$. In this case $q(\gamma)=\Delta^{H \backslash \backslash X}(|\gamma|)$, by definition (1.1). On the other hand, the identification of $H$ with $\pi_{1}\left(H \backslash \backslash X, a_{0}\right)$ is obtained (cf. $[\mathrm{B},(3.6)])$ by restriction to $\pi_{1}\left(H \backslash \backslash X, a_{0}\right)$ of the homomorphism 
$\psi: \pi(H \backslash \backslash X) \rightarrow H$ such that $\psi(s)=s$ for $s \in \mathscr{H}_{a}=H_{a^{T}}$ and $\psi(e)=h_{e}=$ $\left(h_{\partial_{0}} e^{s}\right)\left(h_{\partial_{1}} e^{s}\right)^{-1}$ for $e \in E(H \backslash X)$. It follows that $h_{\gamma}=\psi(|\gamma|)$ for $\gamma$ a closed path at $a_{0}$. Thus it follows from (10) that

$$
\Delta^{H \backslash \backslash X}(h)=\Delta^{H}(h)
$$

whenever $h=|\gamma|, \gamma$ a closed edge path at $a_{0}$. On the other hand, both $\Delta^{H \backslash \backslash X}$ and $\Delta^{H}$ are trivial on all vertex stabilizers in $H$. Thus (11) holds for all $h \in H$, whence the proposition.

From (3.6) and its proof we obtain

(3.7) Corollary. Let $H \leq G$, with closure $\bar{H}$. Let $H^{0} \leq H$ have finite index and act without inversion on $X$ (e.g., $\left.H^{0}=H \cap G^{0} ; c f .(3.1)(1)\right)$.

The following conditions are equivalent.

(a) $\bar{H}$ is unimodular, i.e., $\Delta^{\bar{H}}=1$.

(b) $H^{0} \backslash \backslash X$ is unimodular, i.e., $\Delta^{H^{0} \backslash \backslash X}=1$.

Assume these conditions and let $\mu$ be a Haar measure on $\bar{H}$, rational on compact open subgroups. For $a \in A$ put $N(a)=\mu\left(\bar{H}_{x}^{0}\right)$, where $x$ is any vertex of $X$ over $a$. Then $N$ is well defined and is a vertex ordering of $I\left(H^{0} \backslash \backslash X\right)(c f .(1.3))$.

(3.8) Definition. Under the conditions of (3.7) we say that $H$ is unimodular.

\section{EXISTENCE, CONJUGACY, AND COMMENSURABILITY OF UNIFORM LATTICES}

(4.1) We fix the following notation.

$X=$ a locally finite tree.

$G=\operatorname{Aut}(X)$.

$H \leq G$ is a subgroup acting without inversions on $X$.

$H \backslash \backslash X=(A, \mathscr{H})$, a quotient graph of groups and $p: X \rightarrow A=H \backslash X$ is the quotient morphism.

$G_{H}=\{g \in G \mid g x \in H x, g e \in H e \quad \forall x \in X, e \in E(X)\}$.

Thus, $G_{H}$ is the largest subgroup $H^{\prime}$ of $G$ such that $H^{\prime} \backslash X=H \backslash X \quad(=A)$.

We recall $[\mathrm{B}, \mathrm{I},(5.2)]$

(4.2) Theorem ("Conjugacy Theorem"). If $\Gamma \leq G_{H}$ acts freely on $X$, then there is a $g \in G_{H}$ such that $g \Gamma g^{-1} \leq H$.

(4.3) Definitions. Let $\Gamma \leq G$. We call $\Gamma$ discrete if $\Gamma$ is a discrete subgroup of $G$, i.e., if $\Gamma_{x}$ is finite for all $x \in X$. In this case $\Gamma \backslash \backslash X$ is a graph of finite groups. We call $\Gamma$ an $(X$-)lattice if

$$
\operatorname{Vol}(\Gamma \backslash \backslash X)\left(=\sum_{x \in \Gamma \backslash X} \frac{1}{\left|\Gamma_{x}\right|}\right)<\infty,
$$

and a uniform (X-)lattice if $\Gamma \backslash X$ is finite. 
(4.4) Recall that if $L$ is a locally compact group, then a discrete subgroup $\Gamma \leq L$ is called an $(L$-)lattice if $\Gamma \backslash L$ carries a finite positive $L$-invariant measure, and a uniform ( $L$-)lattice if $\Gamma \backslash L$ is compact. If $L$ admits a lattice, then $L$ must be unimodular, i.e., $\Delta^{L}=1$ (cf. [Rag, Chapter I, Remark 1.9]).

(4.5) Proposition. Suppose that $H$ is a closed subgroup of $G$ and $\Gamma \leq H$ is a discrete subgroup.

(a) If the natural morphism $p: \Gamma \backslash X \rightarrow H \backslash X$ has a finite fiber, e.g., if $\Gamma \backslash X$ is finite, then $\Gamma \backslash H$ is compact. Hence, if $H$ is also discrete, then $\Gamma \backslash H$ is finite.

(b) Suppose that $\Gamma$ is an $X$-lattice, i.e., $\operatorname{Vol}(\Gamma \backslash \backslash X)<\infty$. Then $\Gamma$ is an $H$-lattice, i.e., $\operatorname{Vol}(\Gamma \backslash H)<\infty$, and so $H$ is unimodular.

Proof. (a) Choose $x \in X$ so that $H \cdot x$ consists of finitely many $\Gamma$-orbits. Then $\Gamma \backslash H / H_{x}$ is finite, so $H=\Gamma \cdot S \cdot H_{x}$ for a finite set $S$. Since $S \cdot H_{x}$ is then compact, so also is $\Gamma \backslash H$.

(b) Let $\mu_{H}$ be a left-invariant Haar measure on $H$, and let $p: H \rightarrow \Gamma \backslash H$, $p(h)=[h]$, be the natural projection. There is a unique measure $\mu$ on $\Gamma \backslash H$ such that if $A \subset H$ is measurable and $p \mid A$ is injective, then $\mu(p(A))=\mu_{H}(A)$. For $h \in H$ we then have $\mu(p(A) h)=\mu(p(A h))=\mu_{H}(A h)=\Delta^{H}(h) \mu_{H}(A)$. Thus, $\mu(B h)=\Delta^{H}(h) \mu(B)$ for measurable $B \subset \Gamma \backslash H$.

Let $U$ be a compact open subgroup of $H$, and let $S$ be a set of representatives of $\Gamma \backslash H / U: H=\bigsqcup_{s \in S} \Gamma s U$. Then

$$
\Gamma \backslash H=\coprod_{s \in S} p(s U) .
$$

Now $p(s U)=p\left(s U s^{-1}\right) \cdot s$ and $s U s^{-1} \rightarrow p\left(s U s^{-1}\right)$ is equivalent to $s U s^{-1} \rightarrow$ $\Gamma_{s} \backslash\left(s U s^{-1}\right)$, where $\Gamma_{s}=\Gamma \cap s U s^{-1}$. Therefore,

$$
\mu\left(p\left(s U s^{-1}\right)\right)=\frac{1}{\left|\Gamma_{s}\right|} \cdot \mu_{H}\left(s U s^{-1}\right)=\frac{1}{\left|\Gamma_{s}\right|} \mu_{H}(U) \Delta^{H}(s)^{-1}
$$

and so $\mu(p(s U))=\frac{1}{\mid \Gamma_{s}} \mu_{H}(U)$. Thus,

$$
\mu(\Gamma \backslash H)=\mu_{H}(U) \cdot \sum_{s \in S} \frac{1}{\left|\Gamma_{s}\right|} .
$$

Now let $x \in X$ and take $U=H_{x}$. Then $s U s^{-1}=H_{s x}$ so $\Gamma_{s}=\Gamma_{s x}$. We can identify $H / H_{x}$ with $H \cdot x$, and then the elements $s x(s \in S)$ represent the $\Gamma$-orbits in $H \cdot x$. Thus,

$$
\mu(\Gamma \backslash H)=\mu_{H}\left(H_{x}\right) \cdot \sum_{y \in \Gamma \backslash(H \cdot x)} \frac{1}{\left|\Gamma_{y}\right|} \leq \mu_{H}\left(H_{x}\right) \sum_{y \in \Gamma \backslash X} \frac{1}{\left|\Gamma_{y}\right|}<\infty .
$$

Now for $h \in H$ we have

$$
\mu(\Gamma \backslash H)=\mu((\Gamma \backslash H) \cdot h)=\mu(\Gamma \backslash H) \cdot \Delta^{H}(h),
$$


and so $\Delta^{H}(h)=1$ for all $h$, i.e., $H$ is unimodular, as was to be shown.

We shall see examples below where $G$ itself is discrete, hence a uniform $G$ lattice, yet not an $X$-lattice (Example (4.12)4), and also one where $G$ is a free uniform $X$-lattice (Example (4.12)2).

(4.6) Theorem. Under conditions (4.1), the following conditions are equivalent.

(a) There is a discrete subgroup $\Phi \leq G_{H}$ such that $\Phi \backslash X=H \backslash X \quad(=A)$.

(b) $(U) H$ is unimodular (cf. (3.7)); and $(B D) I(H \backslash \backslash X)$ has bounded denominators (cf. (1.3)).

(c) $I(H \backslash \backslash X)$ admits an integral vertex ordering (cf. (1.3)).

(d) $I(H \backslash \backslash X)$ admits a finite grouping.

Under these conditions, $\Phi$ is a lattice (resp. a uniform lattice) iff $I(H \backslash \backslash X)$ has finite volume (cf. (1.3)) (resp. $H \backslash X$ is finite).

Proof. If $\Phi$ satisfies (a) then $I(\Phi \backslash \backslash X)=I(H \backslash \backslash X)$ so $\Phi \backslash \backslash X$ is a finite grouping of $I(H \backslash \backslash X)$, whence (d). In view of (3.7), the equivalence of (b), (c), and (d) follows from (2.4). Finally, the implication (b) $\Rightarrow(a)$ is contained in the next result.

Note that when $H$ is unimodular and $H \backslash X$ is finite, $I(H \backslash \backslash X)$ automatically has bounded denominators.

(4.7) Theorem. Assume that $H$ is unimodular and that $I(H \backslash \backslash X)$ has bounded denominators, e.g., that $H \backslash X$ is finite. Then there is a subgroup $\Phi^{0} \leq G_{H}$ with the following properties:

(i) $\Phi^{0} \backslash X=H \backslash X$ and $\Phi_{x}^{0}$ is finite cyclic for all $x \in X$.

(ii) If $\Phi \leq G_{H}$ is discrete and $\Phi \backslash X=H \backslash X$, then there is an integer $m$ such that $\left|\Phi_{x}\right|=m\left|\Phi_{x}^{0}\right|$ for all $x \in X$.

(iii) If $\Gamma \leq G_{H}$ acts freely on $X$, then $\Gamma$ is $G_{H}$-conjugate to a subgroup of $H$ and to a subgroup of $\Phi^{0}$.

Suppose that the orders $\left|\Phi_{x}^{0}\right| \quad(x \in X)$ are bounded, e.g., that $H \backslash X$ is finite. Put $M=\operatorname{lcm}_{x \in X}\left|\Phi_{x}^{0}\right|$.

(iv) There is a free subgroup $\Gamma^{0} \leq \Phi^{0}$ of index $M$ and $a g_{0} \in G_{H}$ such that $g_{0} \Gamma^{0} g_{0}^{-1} \leq H$.

(v) With $\Phi$ and $m$ as in (ii), there is a $g \in G_{H}$ such that $g \Gamma^{0} g^{-1} \leq \Phi$, and $\left[\Phi: g \Gamma^{0} g^{-1}\right]=m M$.

Proof. Since $I(H \backslash \backslash X)$ is unimodular with bounded denominators, it follows from (1.4) that there is an integral vertex ordering $N^{0}=N_{\min }$ of $I(H \backslash \backslash X)$ such that any other integral vertex ordering is an integral multiple of $N^{0}$. Let $\mathbf{A}^{0}=\left(A, \mathscr{A}^{0}\right)$ be the finite cyclic grouping of $I(H \backslash \backslash X)$ with $\left|\mathscr{A}_{a}^{0}\right|=N^{0}(a)$ for all $a \in A$, as in (2.3)(2), or (2.4). Choose $a_{0} \in A$ and put $\Phi^{0}=\pi_{1}\left(\mathbf{A}^{0}, a_{0}\right)$, which acts on $\left(\widetilde{\mathbf{A}^{0}, a_{0}}\right)$ with quotient $A$. Since $I\left(\mathbf{A}^{0}\right)=I(H \backslash \backslash X)$, there exist 
bijections

$$
\mathscr{A}_{a / e}^{0} \rightarrow \mathscr{H}_{a / e}
$$

for each $e \in E(A) \quad\left(\partial_{0} e=a\right)$. Since $\left(\widetilde{\mathbf{A}^{0}, a_{0}}\right)$ depends, up to isomorphism over $A$, only on the pointed sets $\mathscr{A}_{a / e}^{0}$ (cf. [B, (1.18)]), and similarly $X=$ $\left(H \backslash \widetilde{\mid X}, a_{0}\right)$ depends only on the $\mathscr{H}_{a / e}$, we can use the bijections (1) to produce an isomorphism of trees $\left(\widetilde{\mathbf{A}^{0}, a_{0}}\right) \rightarrow X$ over $A$. Thus, identifying $\left(\widetilde{\mathbf{A}^{0}, a_{0}}\right)=$ $X$, we obtain $\Phi^{0} \leq G_{H}$ and $\Phi^{0} \backslash X=A$. If $\Phi \leq G_{H}$ is discrete and $\Phi \backslash X=A$, then $\Phi \backslash \backslash X=(A, \mathscr{A})$ is a finite grouping of $I(H \backslash \backslash X)$ so $N(a)=\left|\mathscr{A}_{a}\right|$ defines an integral vertex ordering of $I(H \backslash \backslash X)$; hence $N=m N^{0}$ for some integer $m>0$. If $x \in X$, then $\left|\Phi_{x}\right|=N(p(x))=m N^{0}(p(x))=m\left|\Phi_{x}^{0}\right|$, whence (ii).

Now suppose that $M=\operatorname{lcm}_{x \in X}\left|\Phi_{x}^{0}\right|=\operatorname{lcm}_{a \in A} N^{0}(a)$ is finite. Then by (2.7), $\Phi^{0}$ contains a free subgroup $\Gamma^{0}$ of index $M$, and any other free subgroup has index divisible by $M$. Clearly, $\Gamma^{0}$ acts freely on $X$. If $\Gamma \leq G_{H}=G_{\Phi^{0}}$ acts freely on $X$, then, by the Conjugacy Theorem (4.2), $\Gamma$ is conjugate in $G_{H}$ to a subgroup of $H$ and to a subgroup of $\Phi^{0}$. In particular, $g_{0} \Gamma^{0} g_{0}^{-1} \leq H$ for some $g_{0} \in G_{H}$.

Finally let $\Phi$ be as in (ii): $\Phi \leq G_{H}$ is discrete, $\Phi \backslash X=H \backslash X$, and $\left|\Phi_{x}\right|=$ $m\left|\Phi_{x}^{0}\right|$ for $x \in X$. By the Conjugacy Theorem (4.2) applied to $\Phi$ and $\Gamma^{0}$ we can replace $\Phi$ by a $G_{H^{-}}$-conjugate and assume that $\Gamma^{0} \leq \Phi$. Let $x \in X$. Then $\left|\Gamma^{0} \backslash \Phi^{0} \cdot x\right|=\left|\Gamma^{0} \backslash \Phi^{0} / \Phi_{x}^{0}\right|=\left[\Phi^{0}: \Gamma^{0}\right] /\left|\Phi_{x}^{0}\right|=M /\left|\Phi_{x}^{0}\right|$ since, $\Gamma^{0}$ being free, the finite group $\Phi_{x}^{0}$ acts freely on $\Gamma^{0} \backslash \Phi^{0}$. Since $\Phi \backslash X=H \backslash X=\Phi^{0} \backslash X$ we have $\Phi \cdot x=\Phi^{0} \cdot x$. Thus, by the same reasoning, we have

$$
M /\left|\Phi_{x}^{0}\right|=\mid \Gamma^{0}\left\langle\Phi^{0} \cdot x|=| \Gamma^{0}\left\langle\Phi \cdot x\left|=\left[\Phi: \Gamma^{0}\right] /\right| \Phi_{x}\left|=\left[\Phi: \Gamma^{0}\right] / m\right| \Phi_{x}^{0}\right|,\right.
$$

by (ii). It follows that $\left[\Phi: \Gamma^{0}\right]=m M$. This proves (v), and completes the proof of (4.7).

(4.8) Corollary. Assume that $H$ is unimodular and $H \backslash X$ is finite.

(a) $H$ contains a free uniform lattice $\Gamma^{0}$.

(b) Let $\Gamma \leq H$ be discrete. Assume that $\Gamma$ has "bounded torsion," i.e., that the finite subgroups of $\Gamma$ have bounded orders (e.g., that $\Gamma$ is free, or that $\Gamma$ is finitely generated (cf. [B, II, (8.3)])). Then there is a free subgroup $\Gamma^{\prime}$ of finite index in $\Gamma$ and $a g \in G_{H}$ such that $g \Gamma^{\prime} g^{-1} \leq \Gamma^{0}$.

(c) If $\Gamma_{0}, \Gamma_{1} \leq H$ are uniform lattices, then $g \Gamma_{0} g^{-1}$ and $\Gamma_{1}$ are commensurable for some $g \in G_{H}$.

Proof. Let $\Phi^{0}, \Gamma^{0}$ be as in (4.7), whence (a). Assertion (b) is trivial if $\Gamma$ is finite. Otherwise, the existence of a free $\Gamma_{1}$ of finite index in $\Gamma$ comes from [B, II, (8.3)]. After $G_{H}$-conjugation, using (4.7)(iii), we can assume that $\Gamma_{1} \leq \Phi^{0}$. Then $\Gamma^{\prime}=\Gamma_{1} \cap \Gamma^{0}$ responds to (b). To prove (c), use (b) to get free $\Gamma_{i}^{\prime}$ in $\Gamma_{i}$ 
of finite index $(i=0,1)$ and use (4.7)(iii) again to conjugate $\Gamma_{0}^{\prime}, \Gamma_{1}^{\prime}$ into $\Phi^{0}$. Being uniform lattices, they have finite index in $\Phi^{0}$. Hence, $\Gamma_{0}^{\prime}$ and $\Gamma_{1}^{\prime}$, so also $\Gamma_{0}$ and $\Gamma_{1}$, are commensurable.

(4.9) Corollary. If $H \backslash X$ is finite and $H$ contains a lattice, then $H$ contains a uniform lattice.

It suffices to show that $H$ is unimodular. But $\bar{H}$ (the closure of $H$ in $G$ ) contains a lattice, so $\bar{H}$ is unimodular by $(4.5)(\mathrm{b})$. Hence $H$ is unimodular by (3.8).

(4.10) Corollary (Finite covering criterion). The following conditions are equivalent.

(a) $X$ covers a finite graph.

$\left(a^{\prime}\right) G$ contains a free uniform $X$-lattice.

(b) $(F) G \backslash X$ is finite; and $(U) G$ is unimodular.

Proof. If $X$ covers a finite graph $A$, then $\Gamma=\pi_{1}(A)$ is a free $X$-lattice in $G$. Thus $(\mathrm{a}) \Leftrightarrow\left(\mathrm{a}^{\prime}\right)$. The group $G^{0}$ (cf. (3.1) or [B, II, (6.3)]) of index $\leq 2$ in $G$ acts without inversions on $X$, and satisfies $(F)$ and $(U)$ iff $G$ does. By (4.6), $\left(\mathrm{a}^{\prime}\right)$ for $G^{0} \Leftrightarrow$ (b) for $G^{0}$, whence the corollary.

(4.11) Definition. A locally finite tree $X$ satisfying the conditions of (4.10) will be called a uniform tree.

(4.12) Examples. 1. We shall exhibit a locally finite tree $X$ with $G=\operatorname{Aut}(X)$ such that $G \backslash X$ is finite yet $X$ is not uniform.

Consider the edge-indexed graph

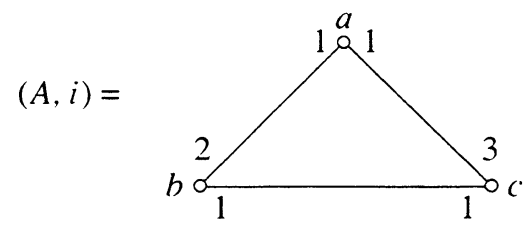

Note that it is not unimodular: $\frac{1}{1} \cdot \frac{3}{1} \cdot \frac{1}{2}=\frac{3}{2} \neq 1$. Let $\mathbf{A}=\mathbf{Z}(A, i)=(A, \mathscr{A})$ be the canonical infinite cyclic grouping of $(A, i)$ (see (2.2)). Thus every vertex and edge group is $\mathbf{Z}$, and $\mathscr{A}_{e} \rightarrow \mathscr{A}_{\partial_{0} e}$ is multiplication by $i(e)$. Let

$$
X=(\widetilde{A, a}), \quad \Gamma=\pi_{1}(\mathbf{A}, a) \cong\left\langle s, t \mid t s^{2} t^{-1}=s^{3}\right\rangle, \quad G=\operatorname{Aut}(X) .
$$

The vertices in the three $\Gamma$-orbits have indices 2 (type a), 3 (type b), and 4 (type c), so the quotient $G \backslash X$ of $A=\Gamma \backslash X$ must coincide with $A$. It follows that $G$ acts without inversion and $(A, i)=I(G \backslash \backslash X)$; hence $G$ is not unimodular; hence $X$ is not uniform, as claimed. 
2. Consider $X=\left(\widetilde{A, a_{0}}\right)$ and $\Gamma=\pi_{1}\left(A, a_{0}\right)$, where (cf. [B, Appendix, Example (A.14)1]),

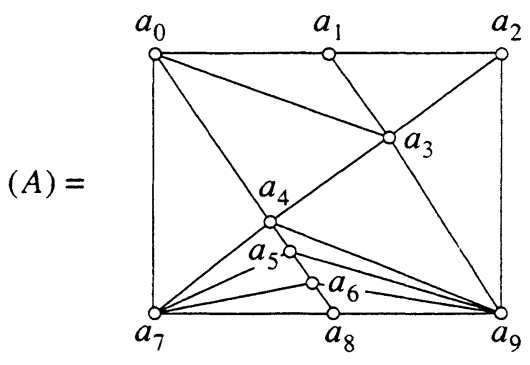

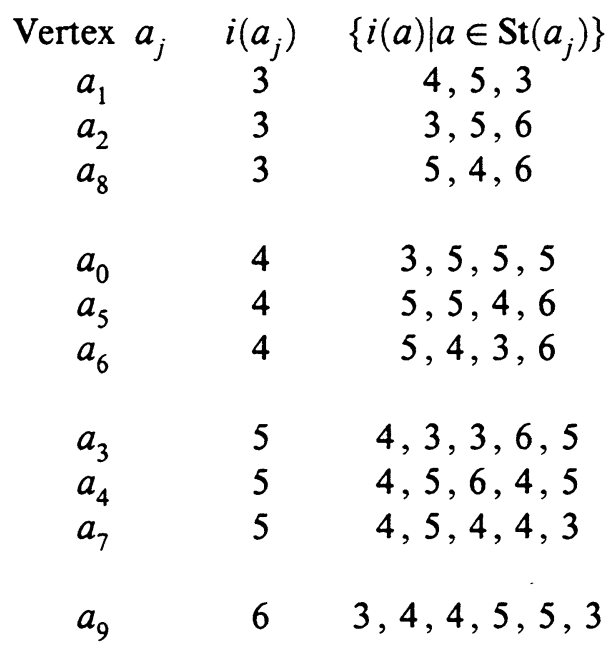

Since the covering $X \rightarrow A$ induces isomorphisms on stars of vertices, it follows from the table that $G=\operatorname{Aut}(X)$ cannot collapse two $\Gamma$-orbits on $X$. Since $A$ is a combinatorial graph (no two edges have the same end points) it follows that $G \backslash X=A$, hence $G=G_{\Gamma}$. But since $\Gamma$ acts freely, $X \rightarrow A$ is a covering and $G_{\Gamma}=\Gamma$ (cf. $\left.[\mathrm{B},(5.5)]\right)$. Thus,

$X$ is a uniform tree such that $G=\operatorname{Aut}(X)$ is a free (uniform) lattice.

Smaller examples, due to Tits, are given in [B, Appendix, Examples (A.14)2, 3].

3. Let

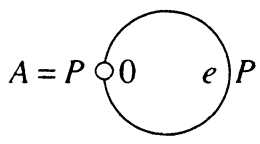


where $P=\widehat{\mathbf{Z}}_{p}$, the $p$-adic integers, and for $x \in P, \alpha_{\bar{e}}(x)=x$ and $\alpha_{e}(x)=p x$. Thus,

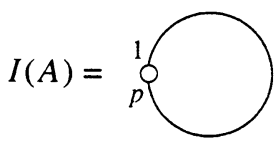

is not unimodular, but its universal cover is the uniform $(p+1)$-homogeneous tree $X_{p+1}$. Put

$$
\left.H=\pi_{1}(\mathbf{A}, 0) \cong\langle P, e| e x e^{-1}=p x \text { for all } x \in P\right\rangle \cong \widehat{\mathbf{Q}}_{p} \rtimes\langle e\rangle,
$$

where $\widehat{\mathbf{Q}}_{p}$ denotes the $p$-adic numbers. Then $H \backslash X_{p+1}=\bigcirc$ is finite, $X_{p+1}$ is uniform, and $H$ is a closed cocompact subgroup of $G=\operatorname{Aut}\left(X_{p+1}\right)$, yet $H$ is not unimodular.

4. Let

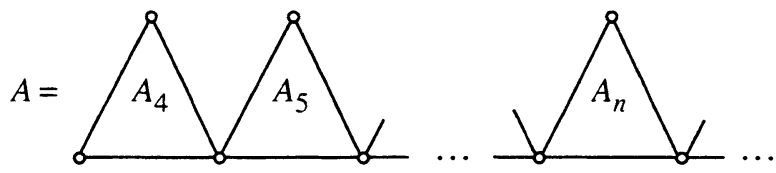

where, for $n \geq 4, A_{n}$ denotes the graph

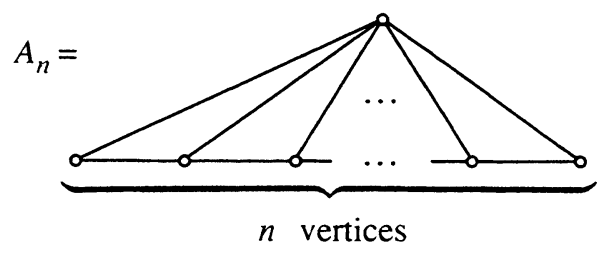

Put $\Gamma=\pi_{1}(A, *)$ and $X=(\widetilde{A, *})$. Then, by combinatorial arguments as in Example 2, one can show that $G=\operatorname{Aut}(X)$ coincides with $\Gamma$. Thus, $X$ admits no lattices, yet $G$, being discrete, is a uniform lattice in itself. Moreover, $G$ acts minimally on $X$ [B, (7.4)].

(4.13) Corollary. Let $X$ be a uniform tree. Let $\Gamma \leq G=\operatorname{Aut}(X)$.

(a) The following conditions are equivalent.

(i) A subgroup of finite index in $\Gamma$ is contained in a uniform lattice on $X$.

(ii) $\Gamma$ is discrete with bounded torsion $(c f .(4.8)(b))$.

(iii) A subgroup of finite index in $\Gamma$ acts freely on $X$. 
(b) If $\Gamma$ acts freely without inversion on $X$, then $\Gamma$ is contained in a uniform lattice on $X$.

Proof. Taking a barycentric subdivision, if necessary, we may assure that $G$ acts without inversion. With $H=G$, let $\Phi^{0}$ be as in (4.7); $\Phi^{0}$ is a uniform lattice. With $\Gamma$ as in (b), by (4.7)(iii) some conjugate of $\Phi^{0}$ contains $\Gamma$, whence (b). For assertion (a), (iii) $\Rightarrow$ (i) follows from (b), (i) $\Rightarrow$ (iii) follows from (4.8)(a) and (c), (iii) $\Rightarrow$ (ii) is trivial, and (ii) $\Rightarrow$ (iii) follows from (4.8)(b).

(4.14) Corollary (Lubotzky [Lub]). Let $H$ be a rank one simple algebraic group over a locally compact nonarchimedean infinite field $F$. Then $H$ contains uncountably many conjugacy classes of cocompact lattices.

Proof. Let $X$ be the (locally finite) Bruhat-Tits tree of $H$ (cf. [BT]). Then $H$ is a closed subgroup of $G=\operatorname{Aut}(X), H$ is unimodular, and $H \backslash X$ is finite. Hence, by (b) $\Rightarrow$ (a) of (4.6), $H$ contains uniform $X$-lattices, which are uniform $H$-lattices by (4.5). It remains to show that these lattices form uncountably many $H$-conjugacy classes.

Let $\Gamma \leq H$ be a free uniform lattice. Let

$$
\Phi=(\varphi,(\gamma)): \Gamma \backslash \backslash X=\mathbf{B}=(B, \mathscr{B}) \rightarrow H \backslash \backslash X=\mathbf{A}=(A, \mathscr{A})
$$

be a corresponding covering morphism (as in $[\mathrm{B}, \S 4]$ ). Since $\Gamma$ acts freely on $X, \mathbf{B}$ is a graph of trivial groups. Let $b_{0} \in B, a_{0}=\varphi\left(b_{0}\right)$. Then we can identify both $\left(\widetilde{\mathbf{B}, b_{0}}\right)$ and $\left(\widetilde{\mathbf{A}, a_{0}}\right)$ with $X$ so that $\Phi_{b_{0}}: \pi_{1}\left(\mathbf{B}, b_{0}\right)$ $\left(=\pi_{1}\left(B, b_{0}\right)\right) \rightarrow \pi_{1}\left(\mathbf{A}, a_{0}\right)$ is identified with the inclusion $\Gamma \leq H$ (loc. cit.). Recall that $(\gamma)$ consists of elements $\gamma_{b} \in \pi_{1}(\mathbf{A}, \varphi(b)) \quad(b \in B)$ and $\gamma_{e}=\gamma_{b} \delta_{e}$ $\left(e \in E(B), \partial_{0} e=b\right)$ with $\delta_{e} \in \mathscr{A}_{\varphi(b)}$. If $b \in B, \varphi(b)=a=\partial_{0} f, f \in E(A)$, then the fact that $\Phi$ is a covering is expressed by the condition that the maps

$$
\Phi_{b / f}: \varphi_{(b)}^{-1}(f) \rightarrow \mathscr{A}_{a} / \alpha_{f} \mathscr{A}_{f}
$$

sending $e$ to $\left[\delta_{e}\right]_{f}$ are all bijective (cf. $\left.[\mathrm{B}, \mathrm{I},(2.6)]\right)$. Recall that $\varphi_{(b)}^{-1}(f)=$ $\left\{e \in E(B) \mid \partial_{0} e=b\right.$ and $\left.\varphi(e)=f\right\}$. Choose (arbitrarily) an element $\varepsilon_{e} \in$ $\alpha_{f} \mathscr{A}_{f} \leq \mathscr{A}_{a}$ whenever $e \in E(B), \partial_{0} e=b, \varphi(e)=f$, and $a=\varphi(b)$. Put $\delta_{e}^{\prime}=\delta_{e} \varepsilon_{e}, \gamma_{e}^{\prime}=\gamma_{b} \delta_{e}^{\prime}$, and $\gamma_{b}^{\prime}=\gamma_{b}$. Then $\Phi^{\prime}=\left(\varphi,\left(\gamma^{\prime}\right)\right): \mathbf{B} \rightarrow \mathbf{A}$ is another morphism, and $\Phi_{b / f}^{\prime}=\Phi_{b / f}$ for all $(b, f)$ as above, so $\Phi^{\prime}$ is again a covering and it defines a new embedding $\Phi_{b_{0}}^{\prime}: \Gamma \rightarrow H$.

Let $T$ be a maximal subtree of $B$. For $b \in B$ let $g_{b}=e_{1} e_{2} \cdots e_{n} \in \pi(B)$, where $\left(e_{1}, e_{2}, \ldots, e_{n}\right)$ is the reduced edge path in $T$ from $b_{0}$ to $b$. For $e \in E(B)$ put $g_{e}=g_{\partial_{0} e} e g_{\partial_{1} e}^{-1} \in \pi_{1}\left(B, b_{0}\right)=\Gamma$. Let $S$ consist of one edge of $E(B)-E(T)$ from each pair $e, \bar{e}$. Then $\Gamma$ is free with basis $\left(g_{e}\right)_{e \in S}$.

For $e \in E(B)$ put $\delta(e)=\delta_{e} \varphi(e) \delta_{\bar{e}}^{-1} \in \pi(\mathbf{A})$. Then $\delta(\bar{e})=\delta(e)^{-1}$, so this defines a homomorphism $\delta: \pi(B) \rightarrow \pi(\mathbf{A})$. Moreover, $\Phi_{b_{0}}: \pi_{1}\left(B, b_{0}\right) \rightarrow$ $\pi_{1}\left(\mathbf{A}, A_{0}\right)$ is given by $\Phi_{b_{0}}(g)=\gamma_{b_{0}} \delta(g) \gamma_{b_{0}}^{-1}$ (cf. [B, (2.9)]). 
Choose $\left(\varepsilon_{e}\right)_{e \in S}$ with $\varepsilon_{e} \in \alpha_{f} A_{f}, f=\varphi(\varepsilon)$. Define $\varepsilon_{e}=1$ for $e \in E(B)-S$. Then define $\Phi^{\prime}=\left(\varphi,\left(\gamma^{\prime}\right)\right)$ as above: $\gamma_{b}^{\prime}=\gamma_{b}, \gamma_{e}^{\prime}=\gamma_{b} \delta_{e}^{\prime}, \delta_{e}^{\prime}=\delta_{e} \varepsilon_{e}$. For the basis elements $g_{e}=g_{\partial_{0} e} e g_{\partial_{1} e}^{-1}(e \in S)$ of $\Gamma$ we have

$$
\Phi_{b_{0}}^{\prime}\left(g_{e}\right)=\gamma_{b_{0}} \delta\left(g_{\partial_{0} e}\right) \delta_{e} \varepsilon_{e} \varphi(e) \delta_{\bar{e}}^{-1} \delta\left(g_{\partial_{1} e}\right)^{-1} \gamma_{b_{0}}^{-1},
$$

which differs from $\Phi_{b_{0}}\left(g_{e}\right)$ only by insertion of the factor $\varepsilon_{e}$. Thus we have a family of distinct lattice embeddings and $\Gamma \rightarrow H$ parametrized by the families $\left(\varepsilon_{e}\right)_{e \in S} \in \prod_{e \in S} \alpha_{f} \mathscr{A}_{f}$, where $f=\varphi(e)$. Since $\mathscr{A}_{f}$ is an open subgroup of $H$, this $F$-manifold has $F$-dimension $d^{r}$, where $d$ is the $F$-dimension of $H$ and $r=|S|$ is the rank of $\Gamma$. The conjugation action of $H$ yields a quotient of $F$-dimension at least $d^{r-1}>0$. Thus, we have uncountably many conjugacy classes of lattice embeddings of $\Gamma$ into $H$. Two of these with the same image differ by composition with an automorphism of $\Gamma$. Since $\operatorname{Aut}(\Gamma)$ is countable, the last assertion of the corollary now follows.

Remarks. 1. Let $\Gamma$ be free on $\left(g_{e}\right)_{e \in S}$ as in the last part of the proof. Then we can identify $\operatorname{Hom}(\Gamma, H)$ with $H^{S}$, and the last part of the proof shows that

$$
\{h \in \operatorname{Hom}(\Gamma, H) \mid h \text { is injective and } h(\Gamma) \text { is an } X \text {-lattice }\}
$$

is open in $\operatorname{Hom}(\Gamma, H)$.

2. The last assertion of (4.14) should be contrasted with Corollary (6.8) below.

(4.15) Corollary ("Commensurability Theorem"). If $\Gamma_{0}, \Gamma_{1}$ are uniform $X$ lattices in $G=\operatorname{Aut}(X)$, then $g \Gamma_{0} g^{-1}$ and $\Gamma_{1}$ are commensurable for some $g \in G$.

Let $H$ be a subgroup of finite index in $G$ acting without inversion on $X$ (e.g., $H=G^{0}$, as in [B, II, (6.3)]). The corollary follows from (4.8)(b) applied to the groups $\Gamma_{i} \cap H$.

(4.16) Corollary (Leighton's "Finite Common Covering Theorem" [L]). If $A_{0}$ and $A_{1}$ are finite graphs with a common covering, then they have a common finite covering.

Let $X$ be the common universal cover of $A_{0}, A_{1}$ and write $A_{i}=\Gamma_{i} \backslash X$ with $\Gamma_{i}=\pi_{1}\left(A_{i}\right)$. After conjugation (4.15) we may assume that $\Gamma_{0}$ and $\Gamma_{1}$ are commensurable. Then $\left(\Gamma_{0} \cap \Gamma_{1}\right) \backslash X$ is the desired common finite cover.

(4.17) Corollary. If $\Gamma_{0}, \Gamma_{1}$ are uniform $X$-lattices, then their commensurators $C_{G}\left(\Gamma_{0}\right)$ and $C_{G}\left(\Gamma_{1}\right)$ are conjugate in $G$.

Recall that $C_{G}(\Gamma)=\left\{g \in G \mid g \Gamma g^{-1}\right.$ and $\Gamma$ are commensurable $\}$. Clearly we have $C_{G}(\Gamma)=C_{G}\left(\Gamma^{\prime}\right)$ if $\Gamma$ and $\Gamma^{\prime}$ are commensurable, and $C_{G}\left(g \Gamma g^{-1}\right)=$ $g C_{G}(\Gamma) g^{-1}$. Hence the corollary follows from (4.15). 
(4.18) Definition. Let $X$ be a uniform tree and $G=\operatorname{Aut}(X)$. Put

$$
\text { Lat }_{u}(X)=\text { the set of uniform } X \text {-lattices. }
$$

Put $C(X)=C_{G}(\Gamma)$ for $\Gamma \in \operatorname{Lat}_{u}(X)$. This is a subgroup of $G$ defined up to conjugacy, which we call "the" commensurability group of $X$.

(4.19) Remarks. 1. $C(X)$ is countable (cf. Appendix B, (B.6)).

2. $C(X)$ contains a conjugate of every uniform lattice on $X(4.15)$.

3. $C(X)=G$ iff $G$ is discrete, because a profinite group is countable iff it is discrete. This occurs, for example, if $X$ is a linear tree.

4. $C(X) \backslash X=G \backslash X$. In view of Remark 2 it suffices to observe that $\Phi^{0} \backslash X=$ $G \backslash X$ for some $\Phi^{0} \in \operatorname{Lat}_{u}(X)$. If $G$ acts without inversion on $X$ this follows from (4.7)(i). Otherwise, pass to the barycentric subdivision $X^{\prime}$ of $X$, where $G$ acts without inversion. By [B, II, (6.5)], $G=\operatorname{Aut}\left(X^{\prime}\right)$ unless $X$ is a linear tree, in which case $C(X)=G($ Remark 3$)$.

(4.20) Conjectures. Let $X$ be a uniform tree, $G=\operatorname{Aut}(X)$.

I. $C(X)$ is dense in $G$.

II. Let $\Gamma \leq G$ and let $Y$ be a subtree of $X$ on which $\Gamma$ acts discretely with finite quotient $\Gamma \backslash Y$. Then there exist $\Gamma^{+} \in \operatorname{Lat}_{u}(X)$ and an injective homomorphism $h: \Gamma \rightarrow \Gamma^{+}$such that $h(g)|Y=g| Y$ for all $g \in \Gamma$.

We shall show below that Conjecture II (even for finite $\Gamma$ ) implies Conjecture I (see (4.23)). We shall verify Conjecture II (and therefore also I) for $X$ homogeneous or bihomogeneous bipartite (Corollary (4.25)).

(4.21) Proposition. Let $X$ be a tree, $x \in X, r>0$, and let $B_{x}(r)=\{y \in$ $X \mid d(x, y) \leq r\}$ and $S_{x}(r)=\{y \in X \mid d(x, y)=r\}$ be the ball and sphere of radius $r$ about $x$, respectively. Let $G=\operatorname{Aut}(X)$ and

$$
G_{x}(r)=\operatorname{Ker}\left(G_{x} \stackrel{\text { restr. }}{\rightarrow} \operatorname{Aut}\left(B_{x}(r)\right)\right)
$$

There is a subgroup $F \leq G_{x}$ such that $G_{x}=G_{x}(r) \rtimes F$. If $X$ is locally finite, then $F$ is finite.

Proof. We can represent $X$ as $B_{x}(r)$ to which we attach a rooted tree $\left(Y_{s}, s\right)$ at each $s \in S_{x}(r)$, and then $G_{x}(r)=\prod_{s \in S_{x}(r)} \operatorname{Aut}\left(Y_{s}, s\right)$. Each $g \in G_{x}$ defines isomorphisms $g_{s}:\left(Y_{s}, s\right) \rightarrow\left(Y_{g s}, g s\right)$ for $s \in S_{x}(r)$. Let $S \subset S_{x}(r)$ be a set of representatives of the $G_{x}$-orbits on $S_{x}(r)$, and put $T_{s}=G_{x} \cdot s$. Choose (arbitrary) isomorphisms to identify $\left(Y_{t}, t\right)$ with $\left(Y_{s}, s\right)$ for all $t \in T_{s}$. Then for $g \in G_{x}$ we can identify $g_{t}:\left(Y_{t}, t\right) \rightarrow\left(Y_{g t}, g t\right)$ with an element of $\operatorname{Aut}\left(Y_{s}, s\right)$. Define $h \in G_{x}(r)$ by $h_{t}=g_{t}^{-1}:\left(Y_{t}, t\right) \rightarrow\left(Y_{t}, t\right)$ for all $t \in S_{x}(r)$.

Then $g h \in F$, where

$$
F=\left\{g \in G_{x} \mid g_{t}=\operatorname{Id}_{(Y, s)} \text { for all } t \in T_{s}, \text { all } s \in S\right\} .
$$

Clearly $F$ is a subgroup of $G_{x}, F \cap G_{x}(r)=\{1\}$, and, as shown above, $G_{x}=G_{x}(r) \cdot F$, whence the first part of the proposition. Since $F$ injects 
into $\operatorname{Aut}\left(B_{x}(r)\right)$ and $B_{x}(r)$ is finite if $X$ is locally finite, $F$ is also finite in this case.

(4.22) Corollary. Let $X$ be a locally finite tree, $G=\operatorname{Aut}(X), C \leq G$. Assume that:

(i) $C \backslash X=G \backslash X$; and

(ii) Let $x \in X, r>0$, and let $F \leq G_{x}$ be a finite subgroup such that $F\left|B_{x}(r)=G_{x}\right| B_{x}(r)$. Then there is a monomorphism $h: F \rightarrow C$ such that $h(F)\left|B_{x}(r)=F\right| B_{x}(r)$.

Then $C$ is dense in $G$.

Proof. Let $x \in X$. By (i) we have $C \cdot x=G \cdot x$ and so $G=C \cdot G_{x}$. It suffices therefore to show that $C_{x}$ is dense in $G_{x}$. The groups $G_{x}(r) \quad(r>0)$ form a base for neighborhoods of the identity in $G_{x}$, so it suffices to show that $G_{x}=C_{x} \cdot G_{x}(r)$ for each $r>0$. Write $G_{x}=G_{x}(r) \rtimes F$ as in (4.21). Apply assumption (ii) to $F$ and $B_{x}(r)$ to obtain $h: F \rightarrow C$ so that $h(F)$ and $F$ coincide on $B_{x}(r)$. Then clearly $h(F) \leq C_{x}$ and $G_{x}=G_{x}(r) \cdot h(F) \leq G_{x}(r) \cdot C_{x}$, thus completing the proof.

Let $X$ be a locally finite tree, $G=\operatorname{Aut}(X)$. Recall from (4.20):

Conjecture II. Let $\Gamma \leq G$ and let $Y$ be a subtree of $X$ on which $\Gamma$ acts discretely with finite quotient $\Gamma \backslash Y$. Then there exist $\Gamma^{+} \in \operatorname{Lat}_{u}(X)$ and an injective homomorphism $h: \Gamma \rightarrow \Gamma^{+}$such that $h(g)|Y=g| Y$ for all $g \in G$.

(4.23) Corollary. If $X$ satisfies Conjecture II for finite groups $\Gamma \leq G$, then $C(X)$ is dense in $G$.

Proof. If suffices to verify (i) and (ii) of (4.22) for $C(X)$. Condition (i) is supplied by Remark 4 of (4.19). For (ii), consider $x \in X, r>0$, and $F \leq$ $G_{x}$ a finite subgroup such that $F\left|B_{x}(r)=G_{x}\right| B_{x}(r)$. From Conjecture II for $\Gamma=F$ and $Y=B_{x}(r)$ we get a monomorphism $h_{0}: F \rightarrow \Gamma^{+} \in \operatorname{Lat}_{u}(X)$ so that $h(f)\left|B_{x}(r)=f\right| B_{x}(r)$ for all $f \in F$. By (4.17), $g_{0} \Gamma^{+} g_{0}^{-1} \leq C(X)$ for some $g_{0} \in G$. Let $h_{1}=\operatorname{ad}\left(g_{0}\right) \circ h_{0}: F \rightarrow C(X)$. Then $g_{0}: B_{x}(r) \rightarrow B_{g_{0} x}(r)$ is equivariant for $h_{1}: F \rightarrow h_{1}(F)$. By condition (i), $c g_{0} x=x$ for some $c \in C(X)$. Put $h=\operatorname{ad}(c) \circ h_{1}: F \rightarrow C(X)$. Then $c g_{0}: B_{x}(r) \rightarrow B_{x}(r)$ is equivariant for $h: F \rightarrow h(F)$. Since $F\left|B_{x}(r)=G_{x}\right| B_{x}(r)$, it follows that the same is true of $h(F)$, whence condition (ii) of (4.22). This completes the proof.

(4.24) Theorem. Let $X$ be a uniform tree, $G=\operatorname{Aut}(X)$. Let $\Gamma \leq G$ and let $Y \subset X$ be a subtree on which $\Gamma$ acts discretely with finite quotient $\Gamma \backslash Y$. Assume that $G$ acts without inversion on $X$ and that $G \backslash X$ is a tree. Then there exist $\Gamma^{+} \in \operatorname{Lat}_{u}(X)$ and a monomorphism $h: \Gamma \rightarrow \Gamma^{+}$such that $h(g)|Y=g| Y$ for all $g \in \Gamma$.

Proof. Since $\Gamma$ is discrete, $\operatorname{Ker}(\Gamma \rightarrow \Gamma \mid Y)$ is finite. Hence, if we enlarge $Y$ to admit all points at distance $\leq r$ from $Y$, we can, for $r$ sufficiently large, make the action of $\Gamma$ on $Y$ effective (= faithful). Assume now that $\Gamma$ acts effectively on $Y$. 
From the inclusion $(\Gamma, Y) \rightarrow(G, X)$ we obtain an immersion of quotient graphs of groups

$$
\Phi=(\phi,(\gamma)): \mathbf{B}=(B, \mathscr{B}) \rightarrow \mathbf{A}=(A, \mathscr{A}),
$$

where $\mathbf{B}=\Gamma \backslash \backslash Y$ and $\mathbf{A}=G \backslash \backslash X$ (cf. [B, I, §4]). Consider the underlying edge-indexed graphs $I(\mathbf{B})=(B, j)$ and $I(\mathbf{A})=(A, i)$. Put

$$
U=\left\{(b, f) \mid b \in B, f \in E(A), \phi(b)=\partial_{0} f\right\}
$$

and, for $(b, f) \in U$,

$$
S(b, f)=\left\{e \in E(B) \mid \partial_{0} e=b, \phi(e)=f\right\} \quad\left(=\phi_{(b)}^{-1}(f)\right) .
$$

The fact that $\Phi$ is an immersion implies that

$$
d_{b}(f):=i(f)-\sum_{e \in S(b, f)} j(e) \geq 0
$$

for all $(b, f) \in U$ (cf. [B, I, (2.8)]).

By assumption, $A=G \backslash X$ is a tree, so each $f \in E(A)$ is a separating edge. We shall schematically write

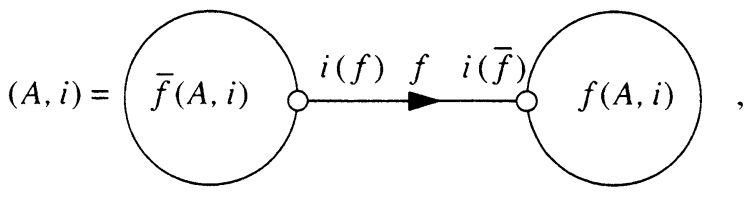

where $f(A, i)$ denotes the portion $(A, i)$ joined to $f$ at $\partial_{1} f\left(=\partial_{0} \bar{f}\right)$, while similarly $\bar{f}(A, i)$ denotes the portion joined to $f$ at $\partial_{0} f$. We now enlarge $(B, j)$ to an edge-indexed graph $\left(B^{+}, j\right)$ as follows. For each $(b, f) \in U$ with $d_{b}(f)>0$ we attach the edge-indexed graph

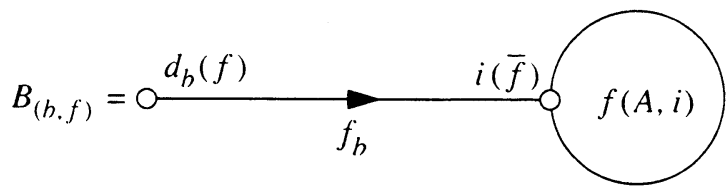

to $(B, j)$ by identifying $\partial_{0} f_{b} \in B_{(b, f)}$ with $b \in B$. Henceforth, we shall view $B_{(b, f)}$, via this identification, with a subgraph of

$$
\left(B^{+}, j\right)=(B, j) \cup \bigcup_{d_{h}(f)>0} B_{(b, f)} .
$$


Next we extend $\phi:(B, j) \rightarrow(A, i)$ to $\phi^{+}:\left(B^{+}, j\right) \rightarrow(A, i)$ as follows: On $B_{(b, f)}, \phi^{+}\left(f_{b}\right)=f$ and $\phi^{+}$induces the inclusion morphism on $f(A, i) \subset$ $B_{(b, f)}$ into $(A, i)$.

Now let $b \in B^{+}, f \in E(A), \phi^{+}(b)=\partial_{0} f$, and put $S^{+}(b, f)=$ $\left\{e \in E\left(B^{+}\right) \mid \partial_{0} e=b\right.$ and $\left.\phi^{+}(e)=f\right\}$. We claim that

$$
i(f)=\sum_{e \in S^{+}(b, f)} j(e) .
$$

For $b \in B$ this has been expressly arranged: Either $S^{+}(b, f)=S(b, f)$ and (3) holds, or else $S^{+}(b, f)=S(b, f) \cup\left\{f_{b}\right\}$, and $j\left(f_{b}\right)=d_{b}(f)$ was chosen to validate (3). If, on the other hand, $b \notin B$, then $b \in f(A, i) \subset B_{\left(b_{0}, f\right)}$ for some $\left(b_{0}, f\right) \in U$. In this case, $\phi^{+}$is defined so as to give a local isomorphism of edge-indexed stars, $\left(\mathrm{St}^{B^{+}}(b), j\right) \rightarrow\left(\mathrm{St}^{A}\left(\phi^{+}(b)\right), i\right)$, so (3) is evident.

Equation (3) says that $\phi^{+}:\left(B^{+}, j\right) \rightarrow(A, i)$ is a covering of edge-indexed graphs, and so they have isomorphic covering trees (cf. [B, I, (2.8)]). We propose now to construct an effective finite grouping $\mathbf{B}^{+}=\left(B^{+}, \mathscr{B}^{+}\right)$of $\left(B^{+}, j\right)$ such that $\mathbf{B}$ is a subgraph of subgroups and, for $e \in E(B), \partial_{0} e=b$, the map $\mathscr{B}_{b} / \alpha_{e} B_{e} \rightarrow \mathscr{B}_{b}^{+} / \alpha_{e}^{+} \mathscr{B}_{e}^{+}$is bijective, so that $\mathbf{B} \subset \mathbf{B}^{+}$is an immersion. This done, for $b_{0} \in B$, we can identify $\left(\widetilde{\mathbf{B}^{+}, b_{0}}\right.$ ) with $X$ (as observed above) and then $\Gamma=\pi_{1}\left(\mathbf{B}, b_{0}\right)$ is embedded in the uniform lattice $\Gamma^{+}=\pi_{1}\left(\mathbf{B}^{+}, b_{0}\right)$ on $X$.

First observe that $\left(B^{+}, j\right)$ is unimodular. This follows since $(B, j)$ is unimodular and $\left(B^{+}, j\right)$ is obtained by attaching edge-indexed trees to $(B, j)$ at a single vertex, so that $\pi_{1}\left(B^{+}\right)=\pi_{1}(B)$. Now it follows from $(2.4)$ that $\left(B^{+}, j\right)$ admits an integral vertex ordering $N: B^{+} \rightarrow \mathbf{N}$ :

$$
\frac{N\left(\partial_{0} e\right)}{j(e)}=\frac{N\left(\partial_{0} \bar{e}\right)}{j(\bar{e})} \in \mathbf{Z}
$$

for all $e \in E\left(B^{+}\right)$. Moreover, $N$ is unique up to a constant factor. Since $b \mapsto\left|B_{b}\right|$ is a vertex ordering of $(B, j)$ (cf. (2.3)), the uniqueness, applied to $(B, j)$, implies that

$$
N(b)=m\left|B_{b}\right| \text { for all } b \in B
$$

for some rational constant $m$. Define $M: B^{+} \rightarrow \mathbf{Q}$ by

$$
M\left(b^{+}\right)= \begin{cases}m & \text { if } b^{+} \in B, \\ N\left(b^{+}\right) /\left|\mathscr{B}_{b}\right| & \text { if } b^{+} \in B_{(b, f)}, \quad(b, f) \in U .\end{cases}
$$

Note that, on $B_{(b, f)}, M$ is a constant multiple of $N$, hence a vertex ordering of $\left(B_{(b, f)}, j\right)$. Multiplying $N$ by a constant we can make $m$ and all of these vertex orderings integral, i.e.,

$$
M(e)=\frac{M\left(\partial_{0} e\right)}{j(e)} \in \mathbf{Z} \text { for all } e \in E\left(B^{+}\right)-E(B) .
$$


For each integer $n \geq 1$ let $\mu_{n}$ denote the group of complex $n$th roots of unity. If $n^{\prime}$ divides $n$, then $\mu_{n^{\prime}} \leq \mu_{n}$. With this notation we define the grouping $\mathbf{B}=\left(B^{+}, \mathscr{B}^{+}\right)$of $\left(B^{+}, j\right)$ as follows. For $b \in B, \mathscr{B}_{b}^{+}=\mathscr{B}_{b} \times \mu_{m}$. If $(b, f) \in U$ and $b^{+} \in B_{(b, f)}$, then $\mathscr{B}_{b^{+}}^{+}=\mathscr{B}_{b} \times \mu_{M\left(b^{+}\right)}$. If $e \in E(B)$, then $\mathscr{B}_{e}^{+}=\mathscr{B}_{e} \times \mu_{m}$ and $\alpha_{e}^{+}: \mathscr{B}_{e} \times \mu_{m} \rightarrow \mathscr{B}_{\partial_{0} e} \times \mu_{m}$ is $\alpha_{e} \times \mathrm{Id}_{\mu_{m}}$. Suppose that $e \in E\left(B_{(b, f)}\right) \quad((b, f) \in U)$. Then $\mathscr{B}_{e}^{+}=\mathscr{B}_{b} \times \mu_{M(e)}$ and $\alpha_{e}^{+}: \mathscr{B}_{b} \times \mu_{M(e)} \rightarrow$ $\mathscr{B}_{b} \times \mu_{M\left(\partial_{0} e\right)}$ is $\operatorname{Id}_{\mathscr{B}_{b}} \times$ inclusion.

Now, as promised, $\mathbf{B}^{+}=\left(B^{+}, \mathscr{B}^{+}\right)$is a finite grouping of $\left(B^{+}, j\right)$ containing $\mathbf{B}=(B, \mathscr{B})$ as a subgraph of subgroups so that $\mathbf{B} \subset \mathbf{B}^{+}$is an immersion. However, we have not taken care to make $\mathbf{B}^{+}$effective. For this we just replace $\mathbf{B}^{+}$by its effective quotient (cf. [B, I, (1.25)]). This does not compromise any of the other conditions above, in particular the immersion $\mathbf{B} \rightarrow \mathbf{B}^{+}$, because, by construction, $\mathbf{B}$ was already effective.

(4.25) Corollary. Let $X=X_{m, n}$, the bihomogeneous bipartite tree with indices $m, n \geq 2$. Then $X$ satisfies Conjectures I and II of (4.20).

Proof. By (4.23), Conjecture II implies Conjecture I, so we need only verify the former.

Case 1. $n \neq m$. Then clearly $G$ acts without inversion on $X$ and $G \backslash X=$ $\circ-\circ$ is a tree, so the corollary follows from (4.24).

Case 2. $m=n=2$. Then $X$ is a linear tree and $G$ itself is a lattice, so we can take $\Gamma^{+}=G$ and $h$ the inclusion in Conjecture II.

Case 3. $m=n>2$. Then $X=X_{n}$, the $n$-homogeneous tree, and $G$ acts with inversion on $X$. Consider the barycentric subdivision $X^{\prime}=X_{n, 2}$. By [B, II, (6.5)], $G=\operatorname{Aut}\left(X^{\prime}\right)$. Thus, we can apply Case 1 to conclude the proof.

(4.26) Remark. Let $X$ be a uniform tree, $G=\operatorname{Aut}(X)$, and $\gamma \in G$. In order for $\gamma$ to belong to some uniform $X$-lattice, $\langle\gamma\rangle$ must be discrete, i.e., either

(i) $\gamma$ is hyperbolic $(l(\gamma)>0)$, or

(ii) $\gamma$ has finite order.

If $\gamma$ is hyperbolic, then $\gamma$ belongs to a uniform lattice, by (4.12)(b). On the other hand, if $\gamma$ has finite order, then, in general, $\gamma$ need not belong to a uniform lattice, nor even to the commensurability group of a uniform lattice. Examples demonstrating this have been produced by Ying-Sheng Liu (Columbia dissertation, in progress). Nonetheless, according to (4.25), $\gamma$ can be "deformed" outside a large finite subtree to an element belonging to a uniform lattice.

\section{VOLUMES, EULER CHARACTERISTICS, AND RANKS}

Let $X$ be a uniform tree (cf. (4.11)), $G=\operatorname{Aut}(X)$, and, for $H \leq G$, put Lat $_{u}(H)=\{\Gamma \leq H \mid \Gamma$ is a uniform lattice on $X\}$. 
For $H=G$ we also write

$$
\operatorname{Lat}_{u}(X)=\operatorname{Lat}_{u}(G) \text {. }
$$

(5.1) Definition. A multiplicative invariant on $\operatorname{Lat}_{u}(X)$ is a function $\varphi: \operatorname{Lat}_{u}(X)$ $\rightarrow \mathbf{R}$ which is invariant, i.e., $\varphi\left(g \Gamma g^{-1}\right)=\varphi(\Gamma)$ for all $\Gamma \in \operatorname{Lat}_{u}(X), g \in G$, and multiplicative, i.e.,

$$
\varphi\left(\Gamma^{\prime}\right)=\left[\Gamma: \Gamma^{\prime}\right] \varphi(\Gamma)
$$

whenever $\Gamma, \Gamma^{\prime} \in \operatorname{Lat}_{u}(X)$ and $\Gamma^{\prime} \leq \Gamma$.

(5.2) Proposition. Let $\varphi: \operatorname{Lat}_{u}(X) \rightarrow \mathbf{R}$ be a multiplicative invariant. Let $\Gamma_{0}, \Gamma_{1}$ $\in \operatorname{Lat}_{u}(X)$.

(a) $\varphi\left(\Gamma_{1}\right)=q \varphi\left(\Gamma_{0}\right)$ for some rational number $q>0$.

(b) Suppose that $\Gamma_{0}$ and $\Gamma_{1}$ are commensurable.

Let $\Gamma^{\prime} \leq \Gamma_{0} \cap \Gamma_{1}$ have finite index. Then

$$
\varphi\left(\Gamma_{1}\right)=\frac{\left[\Gamma_{0}: \Gamma^{\prime}\right]}{\left[\Gamma_{1}: \Gamma^{\prime}\right]} \varphi\left(\Gamma_{0}\right)
$$

(c) Suppose that $\varphi \neq 0$, i.e., for some (hence every) $\Gamma \in \operatorname{Lat}_{u}(X), \varphi(\Gamma) \neq 0$. If $\varphi^{\prime}:$ Lat $_{u}(X) \rightarrow \mathbf{R}$ is any multiplicative invariant, then $\varphi^{\prime}=c \varphi$ for some constant $c \in \mathbf{R}$.

Assertion (b) follows since $\varphi\left(\Gamma^{\prime}\right)=\left[\Gamma_{i}: \Gamma^{\prime}\right] \varphi\left(\Gamma_{i}\right) \quad(i=0,1)$. Assertion (a) follows from (b) and the Commensurability Theorem (4.15). The latter plus (b) further show that $\varphi$ is uniquely determined by $\varphi(\Gamma)$ for any one $\Gamma \in \operatorname{Lat}_{u}(X)$, and this gives assertion (c): $c=\varphi^{\prime}(\Gamma) / \varphi(\Gamma)$ for any $\Gamma$.

(5.3) Proposition. Let $\mu$ be a G-invariant discrete measure on $X$, i.e., $\mu: X \rightarrow$ $\mathbf{R}$ and $\mu(g x)=\mu(x)$ for all $x \in X, g \in G$. For $\Gamma \in \operatorname{Lat}_{u}(X)$ define

$$
\mu(\Gamma)(\text { or } \mu(\Gamma \backslash \backslash X))=\sum_{x \in \Gamma \backslash X} \frac{\mu(x)}{\left|\Gamma_{x}\right|} \text {. }
$$

Then $\mu: \operatorname{Lat}_{u}(X) \rightarrow \mathbf{R}$ is a multiplicative invariant.

Proof. Let $\Gamma \in \operatorname{Lat}_{u}(X), g \in G$. By transport of structure

$$
\mu\left(g \Gamma g^{-1}\right)=\sum_{x \in \Gamma \backslash X} \frac{\mu(g x)}{\left|g \Gamma_{x} g^{-1}\right|}=\mu(\Gamma)
$$

since $\mu$ is $G$-invariant. Suppose that $\Gamma^{\prime} \leq \Gamma$ has finite index. Consider first the case when $\Gamma^{\prime}$ is torsion free. Then

$$
\begin{aligned}
\mu\left(\Gamma^{\prime}\right) & =\sum_{x \in \Gamma^{\prime} \backslash X} \mu(x) \quad\left(\text { all } \Gamma_{x}^{\prime}=1\right) \\
& =\sum_{y \in \Gamma \backslash X} \sum_{x \in \Gamma^{\prime} \backslash \Gamma^{\cdot} \cdot y} \mu(x)=\sum_{y \in \Gamma \backslash X} \mu(y)\left|\Gamma^{\prime} \backslash \Gamma \cdot y\right| \quad(\mu \text { is } \Gamma \text {-invariant }) \\
& =\sum_{y \in \Gamma \backslash X} \mu(y)\left|\Gamma^{\prime} \backslash \Gamma / \Gamma_{y}\right|=\sum_{y \in \Gamma \backslash X} \frac{\mu(y)\left|\Gamma^{\prime} \backslash \Gamma\right|}{\left|\Gamma_{y}\right|}=\left[\Gamma: \Gamma^{\prime}\right] \mu(\Gamma)
\end{aligned}
$$


the last equations following since, with $\Gamma^{\prime}$ torsion free, the finite groups $\Gamma_{y}$ act freely on $\Gamma^{\prime} \backslash \Gamma$.

Now if $\Gamma^{\prime}$ is not torsion free we can choose a torsion free $\Gamma_{1} \leq \Gamma^{\prime}$ of finite index $((4.13)(a))$. By the previous case we have

$$
\left[\Gamma^{\prime}: \Gamma_{1}\right] \mu\left(\Gamma^{\prime}\right)=\left[\Gamma: \Gamma_{1}\right] \mu(\Gamma) .
$$

Dividing by $\left[\Gamma^{\prime}: \Gamma_{1}\right]$ then gives the desired result.

(5.4) Remark. The $G$-invariant measures on $X$ can be identified with $\mathbf{R}^{(G \backslash X)}$, whereas, if $\varphi$ is a nonzero multiplicative invariant on $\operatorname{Lat}_{u}(X)$, the set of multiplicative invariants is the one-dimensional space $\mathbf{R} \cdot \varphi$ (by $(5.2)(c)$ ). Thus, if $|G \backslash X| \neq 1$, i.e., if $X$ is not a homogeneous tree, then the association to an invariant measure $\mu$ of the corresponding multiplicative invariant on $\operatorname{Lat}_{u}(X)$ is not an injective map.

(5.5) Example. Let $\mu_{1}$ denote the unit measure, $\mu_{1}(x)=1$ for all $x \in X$. Then we have

$$
\mu_{1}(\Gamma)=\sum_{x \in \Gamma \backslash X} \frac{1}{\left|\Gamma_{x}\right|}=\operatorname{Vol}(\Gamma \backslash \backslash X)>0 .
$$

(5.6) Corollary. The map $\Gamma \mapsto \operatorname{Vol}(\Gamma \backslash \backslash X)$ is a multiplicative invariant on $\operatorname{Lat}_{u}(X)$. If $\varphi: \operatorname{Lat}_{u}(X) \rightarrow \mathbf{R}$ is any multiplicative invariant, then, for some (unique) constant $c_{\varphi} \in \mathbf{R}$,

$$
\varphi(\Gamma)=c_{\varphi} \cdot \operatorname{Vol}(\Gamma \backslash \backslash X) \text { for all } \Gamma \in \operatorname{Lat}_{u}(X) .
$$

(5.7) Example. Recall that for $x \in X$,

$$
i(x)=|\operatorname{St}(x)|=\operatorname{Card}\left\{e \in E(X) \mid \partial_{0} e=x\right\} .
$$

Define the Euler-Poincaré measure $\mu_{\mathrm{EP}}$ on $X$ by $\mu_{\mathrm{EP}}(x)=1-i(x) / 2$. Thus, for $\Gamma \in \operatorname{Lat}_{u}(X)$,

$$
\mu_{\mathrm{EP}}(\Gamma)=\sum_{x \in \Gamma \backslash X}\left(\frac{2-i(x)}{2}\right) /\left|\Gamma_{x}\right| .
$$

(5.8) Proposition. (a) For $\Gamma \in \operatorname{Lat}_{u}(X)$,

$$
\mu_{\mathrm{EP}}(\Gamma)=\chi(\Gamma),
$$

the rational Euler characteristic of $\Gamma$ (cf. [S2]).

(b) There is a constant $\kappa(X) \in \mathbf{Q}$ such that

$$
\chi(\Gamma)=\kappa(X) \cdot \operatorname{Vol}(\Gamma \backslash \backslash X) \quad \text { for all } \Gamma \in \operatorname{Lat}_{u}(X) .
$$

Proof. (a) Since both terms define multiplicative invariants of $\Gamma$ (see [S2], for $\chi$ ) it suffices to show that they agree for a single $\Gamma$, by (5.2). Thus we can 
assume that $\Gamma$ is free, in which case, putting $A=\Gamma \backslash X$, we have

$$
\begin{aligned}
\chi(\Gamma) & =\chi(A)=|A|-\frac{1}{2}|E(A)|=\sum_{a \in A} 1-\frac{1}{2} \sum_{e \in E(A)} 1 \\
& =\sum_{a \in A}\left(1-\frac{1}{2} \sum_{\partial_{0} e=a} 1\right)=\sum_{a \in A}\left(1-\frac{i(a)}{2}\right)=\mu_{\mathrm{EP}}(\Gamma) .
\end{aligned}
$$

Assertion (b) follows from (5.2)(c) and (5.5).

(5.9) Corollary. If $X$ covers a finite graph $A$, then

$$
\frac{|E(A)|}{|A|}=2(1-\kappa(X))
$$

which is independent of $A$.

Proof. Write $A=\Gamma \backslash X$ with $\Gamma$ a free lattice. Then

$$
|A|-\frac{1}{2}|E(A)|=\chi(A)=\chi(\Gamma)=\kappa(X) \cdot \operatorname{Vol}(\Gamma \backslash \backslash X)=\kappa(X) \cdot|A|,
$$

whence the formula.

(5.10) Definitions. Let $A$ be a finite connected graph, $a_{0} \in A, \Gamma=\pi_{1}\left(A, a_{0}\right)$, and $X=\left(\widetilde{A, a_{0}}\right)$. The rank $r$ of the free group $\Gamma$ will be called the rank of $A$; it is also the first Betti number of $A$. Thus

$$
\operatorname{rank}(A)=1-\chi(A)=1-\chi(\Gamma) .
$$

(5.11) Examples. 0. $\operatorname{rank}(A)=0$ iff $A$ is a tree (i.e., $X=A$, or $\Gamma=1$ ).

1. $\operatorname{rank}(A)=1$ iff $\Gamma$ is infinite cyclic. In this case $A$ is "virtually cyclic," in the sense that $A$ is obtained from a cyclic graph $(\mathbf{Z} / n \mathbf{Z}$, with edges $(i, i \pm 1)$ or a loop if $n=1$ ) by attaching a finite tree at each vertex. Then $X$ is "virtually linear", in the sense that $X$ is obtained from a linear tree $(\mathbf{Z}$, with edges $(i, i \pm 1))$ by attaching finite trees to each vertex so that these finite trees are periodically isomorphic (with period $n$ ).

2. If $\operatorname{rank}(A)>1$ we call $A$ a hyperbolic finite graph, and $X$ a hyperbolic uniform tree.

(5.12). Since

$$
1-r=\chi(\Gamma)=\kappa(X) \operatorname{Vol}(\Gamma \backslash \backslash X)=\kappa(X)|A|,
$$

we conclude that, in the respective cases $0,1,2$ above, we have $\kappa(X)=1 /|X|>$ $0, \kappa(X)=0$, and $\kappa(X)<0$, respectively. Moreover the number of (open) ends of $X$ is 0,2 , and $\infty$, respectively.

Thus we have the following trichotomy for uniform trees $X$. 


\begin{tabular}{|l|c|c|c|}
\hline Type of $X$ & finite & $\begin{array}{c}\text { virtually } \\
\text { linear }\end{array}$ & hyperbolic \\
\hline $\begin{array}{l}\text { Types of finite } \\
\text { graphs covered } \\
\text { by } X\end{array}$ & $\begin{array}{c}\text { finite tree } \\
\text { (rank 0) }\end{array}$ & $\begin{array}{c}\text { virtually cyclic } \\
\text { (rank 1) }\end{array}$ & $\begin{array}{c}\text { hyperbolic } \\
(\text { rank } \geq 2)\end{array}$ \\
\hline$\kappa(X)$ & $\kappa(X)=\frac{1}{|X|}>0$ & $\kappa(X)=0$ & $\kappa(X)<0$ \\
\hline $\begin{array}{l}\text { Number of open } \\
\text { ends of } X\end{array}$ & 0 & 2 & $\infty$ \\
\hline $\begin{array}{l}\text { Types of uniform } \\
\text { lattices on } X\end{array}$ & finite & $\begin{array}{c}\text { virtually } \\
\text { infinite cyclic }\end{array}$ & $\begin{array}{c}\text { virtually free } \\
\text { nonabelian }\end{array}$ \\
\hline
\end{tabular}

(5.13) Examples. 1. Let $X=X_{p, q}$, the bihomogeneous bipartite tree with indices $p$ and $q$. The free product $\Gamma=(\mathbf{Z} / p \mathbf{Z}) *(\mathbf{Z} q \mathbf{Z})$ acts on $X$ with

$$
\Gamma \backslash \backslash X=(\mathbf{Z} / p \mathbf{Z}) \quad 1 \quad(\mathbf{Z} / q \mathbf{Z}) .
$$

Thus,

$$
\chi(\Gamma)=\left(\frac{1}{p}+\frac{1}{q}-1\right)=\kappa(X) \cdot \operatorname{Vol}(\Gamma \backslash \backslash X)=\kappa(X)\left(\frac{1}{p}+\frac{1}{q}\right)
$$

so

$$
\kappa(X)=1-\left(\frac{1}{p}+\frac{1}{q}\right)^{-1}=\frac{p+q-p q}{p+q} .
$$

Thus,

$$
\kappa\left(X_{p, q}\right)=\frac{p+q-p q}{p+q} .
$$

When $p=q, X_{p, q}$ is just the $p$-homogeneous tree $X_{p}$, and we obtain

$$
\kappa\left(X_{p}\right)=1-p / 2 \text {. }
$$

2. The following example shows that uniform lattices on virtually linear trees need not be commensurable. Consider the virutally cyclic graph

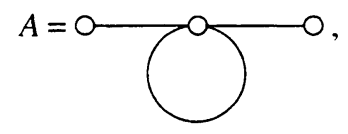

with universal cover $X$ :

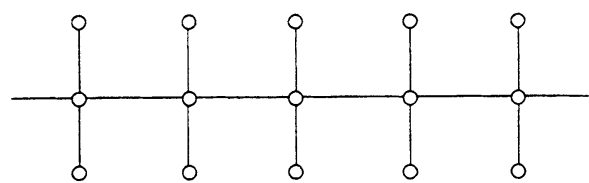


Then $G=\operatorname{Aut}(X)$ is isomorphic to the semidirect product

$$
(\mathbf{Z} / 2 \mathbf{Z})^{\mathbf{Z}} \rtimes D_{\infty}
$$

The dihedral group $D_{\infty}=\left\langle t, \sigma \mid \sigma^{2}=1, \sigma t \sigma^{-1}=t^{-1}\right\rangle$ acts on the horizontal axis, identified with $\mathbf{Z}$, by $t(n)=n+1$ and $\sigma(n)=-n$. The action defining the semidirect product $(*)$ is defined, for $g \in D_{\infty}$ and $\varepsilon \in(\mathbf{Z} / 2 \mathbf{Z})^{\mathbf{Z}}$, by $g(\varepsilon)(n)=\varepsilon\left(g^{-1}(n)\right)$. If, for $h \in \mathbf{Z}$, we write $\varepsilon_{h}(n)=\varepsilon(n-h)$, then $t^{h}(\varepsilon)=\varepsilon_{h}$. Putting $g_{\varepsilon}=(\varepsilon, t) \in(\mathbf{Z} / 2 \mathbf{Z})^{\mathbf{Z}} \rtimes D_{\infty}$, we then have

$$
g_{\varepsilon}^{N}=\left(\varepsilon+t(\varepsilon)+\cdots+t^{N-1}(\varepsilon), t^{N}\right)=\left(\varepsilon+\varepsilon_{1}+\cdots+\varepsilon_{N-1}, t^{N}\right)
$$

for $N>0$. Choose $\varepsilon$ so that $\varepsilon+\varepsilon_{1}+\cdots+\varepsilon_{N-1} \neq 0$ for all $N>0$, e.g., $\varepsilon(0)=1$ and $\varepsilon(n)=0$ for $n \neq 0$. Then $g_{\varepsilon}^{N} \neq g_{0}^{N}$ for all $N>0$. Thus $\left\langle g_{0}\right\rangle$ and $\left\langle g_{\varepsilon}\right\rangle$ are uniform $X$-lattices (with quotient $A$ ) which are not commensurable.

(5.14) Definition. Let $X$ be a uniform tree. Define

$$
r(X)=\text { the minimum rank of a free lattice on } X
$$

and

$v(X)=\operatorname{minimum}|A|, \quad$ where $A$ varies over finite graphs covered by $X$.

(5.15) Examples. 0. If $X$ is finite, then $\Gamma=1$ is the unique free lattice on $X$, $A=X$ is the unique graph covered by $X$, and so $r(X)=0, v(X)=|X|$.

1. $r(X)=1$ iff $X$ is virtually linear. If $X$ is actually linear, then $v(X)=1$; take

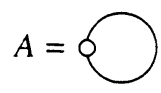

Otherwise $v(X)>1$.

2. Suppose that $r(X)>1$, i.e., that $X$ is hyperbolic, and hence $\kappa(X)<0$. Let $\Gamma$ be a free lattice on $X$ of rank $r$ and $A=\Gamma \backslash X$. Then $1-r=\chi(\Gamma)=$ $\kappa(X)|A|$, so we see that $r$ and $|A|$ are simultaneously minimal: $r=r(X)$ iff $|A|=v(X)$.

3. $v(X)=1$ iff $X$ covers a bouquet $A_{m}$ of $m$ circles, for some $m \geq 0$. In this case $X=X_{2 m}$, the $2 m$-homogeneous tree, so we see that

$$
v\left(X_{2 m}\right)=1 \text { and } r\left(X_{2 m}\right)=m \text {. }
$$

4. A connected graph with two vertices must be of the form

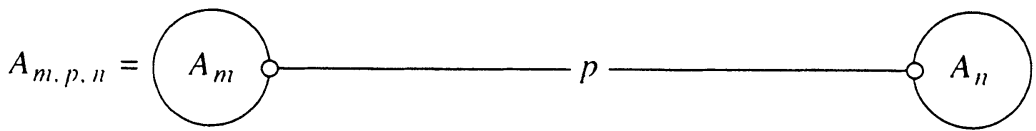


i.e., bouquets of $m$ and $n$ circles, respectively, joined by $p$ (geometric) edges; here $m, n \geq 0$ and $p>0$. Let $X=X_{m, p, n}$ denote the universal cover of $A_{m, p, n}$.

Case (i). $m=n$. Put $N=2 m+p=2 n+p$. Then $X=X_{N}$. For $N$ even (i.e., $p$ even) we saw (Example 3) above that $v(X)=1$ and $r(X)=N / 2$. If $N$ (and $p$ ) are odd, then we must have $v(X)=2$ and so $r(X)=\operatorname{rank}\left(A_{m, p, n}\right)=$ $m+p+n-1=N-1$. Thus:

For $N$ odd,

$$
v\left(X_{N}\right)=2 \text { and } r\left(X_{N}\right)=N-1 .
$$

Case (ii). $m \neq n$. Then it is easily seen that $X_{m, p, n}$ and $X_{m^{\prime}, p^{\prime}, n^{\prime}}$ are isomorphic iff $p=p^{\prime}$ and $\{m, n\}=\left\{m^{\prime}, n^{\prime}\right\}$. We have $v\left(X_{m, p, n}\right)=2$ and $r\left(X_{m, p, n}\right)=m+p+n-1$.

5. Let $X=X_{m, n}$, the bihomogeneous bipartite tree with indices $m \neq n$. The group $\Gamma=(\mathbf{Z} / m \mathbf{Z}) *(\mathbf{Z} / n \mathbf{Z})$ is a uniform lattice on $X$ with edge-indexed quotient graph $(A, i)=\circ \stackrel{m}{n} \circ$. Clearly also $A=G \backslash X$, where $G=\operatorname{Aut}(X)$, so $G$ acts without inversion on $X$ and $G=G_{\Gamma}$. It follows therefore from the Conjugacy Theorem (4.2) that every free lattice on $X$ is conjugate to a subgroup of $\Gamma$.

Let $d=\operatorname{gcd}(m, n), m=m_{0} d, n=n_{0} d$, and $M=\operatorname{lcm}(m, n)=m_{0} n_{0} d$. According to (2.8), $\Gamma$ contains a free subgroup $\Gamma^{0}$ of index $M$, and every other free subgroup has index divisible by $M$. If $r=\operatorname{rank}\left(\Gamma^{0}\right)$, then

$$
1-r=\chi\left(\Gamma^{0}\right)=M \cdot \chi(\Gamma)=M \cdot\left(\frac{1}{m}+\frac{1}{n}-1\right) .
$$

It follows that $r$ is minimal, i.e., $r=r(X)$, so

$$
r(X)=1-M\left(\frac{1}{m}+\frac{1}{n}-1\right)=1-m_{0}-n_{0}+m_{0} n_{0} d .
$$

Recall from (5.13) that

$$
\kappa(X)=\frac{m+n-m n}{m+n}=\frac{m_{0}+n_{0}-m_{0} n_{0} d}{m_{0}+n_{0}}
$$

and so, since $1-r(X)=\kappa(X) v(X)$,

$$
\begin{aligned}
v(X) & =\kappa(X)^{-1}(1-r(X)) \\
& =\left(\frac{m_{0}+n_{0}-m_{0} n_{0} d}{m_{0}+n_{0}}\right)^{-1}\left(m_{0}+n_{0}-m_{0} n_{0} d\right)=m_{0}+n_{0} .
\end{aligned}
$$

In summary: For $m \neq n$,

$$
r\left(X_{m, n}\right)=1-m_{0}-n_{0}+m_{0} n_{0} d
$$

and

where $d=\operatorname{gcd}(m, n), m=m_{0} d, n=n_{0} d$. 
(In case $m=n$, so $X_{m, n}=X_{m}$, this still gives the correct answers for $m$ odd (Example 4(i)), but not for $m$ even.)

A minimal graph $A$ covered by $X_{m, n}$ is obtained as follows: $A=M \amalg$ $N$, where $M$ and $N$ have $m_{0}$ and $n_{0}$ elements, respectively. Connect each element of $M$ to each element of $N$ by $d$ (geometric) edges. Example: $m=6$, $n=4$, so $d=2$ :

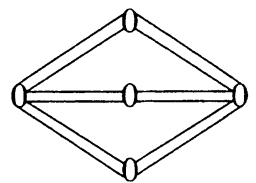

(5.16). Let $X$ be a uniform tree and $\Phi \in \mathrm{Lat}_{u}(X)$. Put $m(\Phi)=\operatorname{lcm}|F|$, where $F$ varies over all finite subgroups of $\Phi$. Assume that $\Phi$ acts without inversion on $X$. (This can be achieved either by subdividing $X$ or by passing to a subgroup of index $\leq 2$ in $\Phi$.) Then (cf. [B, II, (7.3)]) every finite subgroup of $\Phi$ fixes a vertex of $X$; hence

$$
m(\Phi)=\operatorname{lcm}_{x \in \Phi \backslash X}\left|\Phi_{x}\right|
$$

According to (2.8), $\Phi$ contains a free subgroup $\Gamma$ of index $m(\Phi)$. If $r=$ $\operatorname{rank}(\Gamma)$, then $1-r=m(\Phi) \chi(\Phi)$, so $r=r(\Phi)$ is independent of the choice of $\Gamma$, and

$$
1-r(\Phi)=m(\Phi) \chi(\Phi) \text {. }
$$

If $\Gamma^{\prime} \leq \Phi$ is free of rank $r^{\prime}$ and index $m^{\prime}$, then $m^{\prime}=n m$ for some integer $n>0$ (loc. cit.). Hence $1-r^{\prime}=\chi\left(\Gamma^{\prime}\right)=n m \chi(\Phi)=n(1-r)$, so $r^{\prime}=1+n(r-1)$. Thus either $r^{\prime}=r=0$ or 1 or else $r>1$ (i.e., $X$ is hyperbolic, cf. (5.11)(2)) and $r^{\prime} \geq r$. It follows that

$$
r(\Phi)=\text { the least rank of a free subgroup of finite index in } \Phi
$$

and

$$
m(\Phi)=\text { the least index of a free subgroup of } \Phi \text {. }
$$

If $\Phi \backslash X=G \backslash X$, where $G=\operatorname{Aut}(X)$, then we have $G=G_{\Phi}$, so it follows from the Conjugacy Theorem (4.2) that every free lattice on $X$ is conjugate to a subgroup of $\Phi$. Therefore:

If $\Phi \backslash X=G \backslash X$, then

$$
r(X)=r(\Phi) \text { and } v(X)=m(\Phi) \operatorname{Vol}(\Phi \backslash \backslash X) .
$$

(5.17) Example. If $\Gamma$ is a maximal free lattice on $X$, then it is not necessarily the case that $\operatorname{rank}(\Gamma)=r(X)$. For example, let $\Phi=G_{0} * G_{1}$, where $\left|G_{i}\right|=m_{i}$ and $m_{0} \neq m_{1}$. Then $\Phi$ is a uniform lattice on $X=X_{m_{0}, m_{1}}$ with edge-indexed quotient $\stackrel{m_{0} m_{1}}{\circ}$, and clearly $\Phi \backslash X=G \backslash X$, where $G=\operatorname{Aut}(X)$. Hence every free lattice on $X$ is conjugate to a subgroup of $\Phi$ (Conjugacy Theorem $(4.2)), r(X)=r(\Phi)$, and

$$
v(X)=m(\Phi) \operatorname{Vol}(\Phi \backslash \backslash X)=\left(\frac{m_{0} m_{1}}{d}\right)\left(\frac{1}{m_{0}}+\frac{1}{m_{1}}\right)=\frac{m_{0}+m_{1}}{d}
$$


where $d=\operatorname{gcd}\left(m_{0}, m_{1}\right)$. Let

$$
\Phi^{\prime}=\operatorname{Ker}\left(\Phi=G_{0} * G_{1} \rightarrow G_{0} \times G_{1}\right),
$$

a free subgroup of index $m_{0} m_{1}$. Let $\Gamma$ be a maximal free subgroup of $\Phi$ containing $\Phi^{\prime}$, hence a maximal free lattice on $X$. Suppose that $\operatorname{rank}(\Gamma)=$ $r(X)$, and hence $|\Phi / \Gamma|=m(\Phi)=m_{0} m_{1} / d$. The transitive action of $\Phi$ on $\Phi / \Gamma$ factors through $G_{0} \times G_{1}$, and each $G_{i}$ acts freely. Put $T=G_{1} \backslash(\Phi / \Gamma)$. Then $T$ is a transitive $G_{0}$-set (since the actions of $G_{0}$ and $G_{1}$ on $\Phi / \Gamma$ commute) and $|T|=\frac{1}{m_{1}}|\Phi / \Gamma|=m_{0} m_{1} / m_{1} d=m_{0} / d$. Hence the isotropy groups of $G_{0}$ on $T$ have order $d=\operatorname{gcd}\left(m_{0}, m_{1}\right)$.

Now to prevent this, choose a group $G_{0}$ of order $m_{0}$ with a divisor $d$ of $m_{0}$ such that $G_{0}$ has no subgroup of order $d$. (For example, $G_{0}=V \rtimes\langle t\rangle$, where $V$ is elementary of order $4, t^{3}=1$, and $t$ acts as an automorphism of order 3 on $V$; then $\left|G_{0}\right|=12$ and $G_{0}$ has no subgroup of order $d=6$.) Choose any integer $m_{1}^{\prime}$ relatively prime to $m_{0} / d$, and any group $G_{1}$ of order $m_{1}=d m_{1}^{\prime}$. Then $\Phi=G_{0} * G_{1}$ gives the desired example: A maximal free subgroup $\Gamma$ of $\Phi$ containing $\Phi^{\prime}$ is a maximal free lattice on $X$, yet $\operatorname{rank}(\Gamma)$ must be $>r(X)$.

(5.18). Let $A$ be a finite graph. Recall that, for $a \in A$,

$$
i(a)=\left|\left\{e \in E(A) \mid \partial_{0} e=a\right\}\right| \text {. }
$$

Put $v_{n}(A)=|\{a \in A \mid i(a)=n\}|$. Then

$$
|A|=\sum_{n \geq 0} v_{n}(A) \text { and }|E(A)|=\sum_{n \geq 0} n v_{n}(A) .
$$

Hence,

$$
\chi(A)=|A|-\frac{1}{2}|E(A)|=\frac{1}{2} \sum_{n \geq 0}(2-n) v_{n}(A) .
$$

Suppose $A$ is connected of $\operatorname{rank} r(A)=\operatorname{rank}\left(\pi_{1}(A)\right)$. Since $\chi(A)=1-r(A)$ we have

$$
r(A)=1+\frac{1}{2} \sum_{n \geq 0}(n-2) v_{n}(A) .
$$

If $v_{0}(A)>0$, then $A$, being connected, is just a single vertex. Thus, if $A$ is connected and $E(A) \neq \varnothing$ we have

$$
r(A)=1+\frac{1}{2}\left(-v_{1}(A)+v_{3}(A)+2 v_{4}(A)+3 v_{5}(A)+\cdots\right) .
$$

(5.19) Proposition. Among connected finite graphs $A$ with no vertices of index $\leq 2$, there are only finitely many of a given rank $r$.

In fact, from (5.18) above we have $r=1+\frac{1}{2}\left(v_{3}(A)+2 v_{4}(A)+3 v_{5}(A)+\cdots\right) \geq$ $1+\frac{1}{2}|A|$, so $|A| \leq 2(r-1)$. Further, $1-r=|A|-\frac{1}{2}|E(A)|$ so $|E(A)|=$ $2(|A|+(r-2)) \leq 6(r-1)$. 
(5.20) Example. For $r=2$ we must have $|A| \leq 2$. The possibilities are as follows:
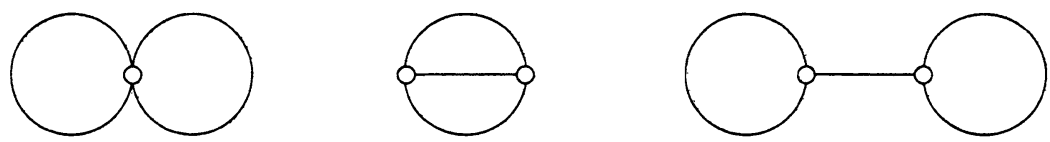

(5.21) Corollary. Given an integer $r>0$, there are (up to isomorphism) only finitely many uniform trees $X$ of rank $r(X)=r$ having no vertices of index $\leq 2$.

(5.22) Remark. For an arbitrary finite connected graph $A$, we can "trim" $A$ as follows. If $A$ has at most one (geometric) edge, leave it be. Otherwise let $P(A)$ denote the result of pruning away all terminal vertices (index 1) (along with their incident edges). After finitely many steps, $P^{n}(A)=\operatorname{Core}(A)$ either has no more terminal vertices, or else has at most one edge. If all vertices of $\operatorname{Core}(A)$ have index 2 , then $A$ must be a circuit $(\mathbf{Z} / n \mathbf{Z}$, with edges $(i, i \pm 1))$, and we then suppress all but one of the vertices to obtain a loop. If $\operatorname{Core}(A)$ has branch points (vertices of index $\geq 3$ ), then we suppress all vertices of index 2 , amalgamating the two incident edges at each such vertex. The resulting graph $T(A)$ now has no vertices of index 1 , unless $T(A)=\circ-\circ$, and no vertices of index 2 unless $T(A)=\odot$. Moreover, it is evident that $T(A)$ has the same homotopy type as $A$, so $\operatorname{rank}(T(A))=\operatorname{rank}(A)$. For fixed $r=\operatorname{rank}(A)$, Proposition (5.19) allows only finitely many possibilities for $T(A)$. On the other hand, $A$ can be obtained from $T(A)$ by the following two operations.

1. Subdivide various edges of $T(A)$, to obtain $B$.

2. At each vertex of $B$, graft a finite tree.

In passing to the universal covers, these correspond to similar operations on the covering trees.

\section{FINITENESS PROPERTIES}

As in $\S 5, X$ denotes a uniform tree, $G=\operatorname{Aut}(X)$, and $\operatorname{Lat}_{u}(X) \quad\left(=\operatorname{Lat}_{u}(G)\right)$ denotes the set of uniform lattices $\Gamma$ on $X$.

(6.1) Proposition. Let $\Gamma \in \operatorname{Lat}_{u}(X)$, with centralizer $Z=Z_{G}(\Gamma)$. Assume that $X$ is not finite.

(a) $\Gamma$ and $G$ have the same minimal subtree: $X_{\Gamma}=X_{G}$ (cf. [B, II, (7.5)]).

(b) Either $Z$ is finite and acts trivially on $X_{G}$, or else $X$ is virtually linear (cf. $(5.11) 1)$ and $Z$ is a lattice, commensurable with $\Gamma$.

Proof. (a) After subdividing $X$, if necessary, we may assume that $G$ acts without inversion on $X$. By (4.7), $G \backslash X=\Phi^{0} \backslash X$ for some $\Phi^{0} \in \operatorname{Lat}_{u}(X)$. Then by [B, II, (7.8)], $X_{G}=X_{\Phi^{0}}$. Let $\Gamma \in \operatorname{Lat}_{u}(X)$. By (4.8), $g \Phi^{0} g^{-1}$ and $\Gamma$ are commensurable for some $g \in G$. By [B, (7.7)] (which is applicable since $X$ is not finite), $X_{\Gamma}=X_{g \Phi^{0} g^{-1}}$. But $X_{g \Phi^{0} g^{-1}}=g X_{\Phi^{0}}=g X_{G}=X_{G}$, whence (a). 
(b) We first claim that $Z$ is discrete. Let $Y \subset X$ be a finite subtree such that $\Gamma \cdot Y=X$. If $\alpha \in Z$ fixes all vertices of $Y$, then the tree $X_{\alpha}$ of fixed points of $\alpha$ contains $Y$ and is $\Gamma$-invariant, hence $X_{\alpha}=X$, i.e., $\alpha=1$. Hence $Z$ is discrete, as claimed.

Moreover, for $y \in Y$ and $\gamma \in \Gamma$ we have $Z_{\gamma y}=\gamma Z_{y} \gamma^{-1}=Z_{y}$, so $Z$ has only finitely many vertex stabilizers $(Y$ being finite and $X=\Gamma \cdot Y)$. It follows therefore (cf. [B, (7.2)]) that if $l(Z)=0$, then $Z$ has a fixed points; hence $Z$ is finite.

Suppose finally that $Z$ is infinite, so that $l(\alpha)>0$ for some $\alpha \in Z$. Then the $\alpha$-axis $X_{\alpha}$ is $\Gamma$-invariant, so $X_{\alpha}$ contains, therefore equals, $X_{\Gamma}$. Thus, $X$ is virtually linear and $\Gamma$ is virtually cyclic. Let $\gamma_{0} \in \Gamma$ generate a subgroup $\left\langle\gamma_{0}\right\rangle$ of finite index; thus $\left\langle\gamma_{0}\right\rangle$ is a lattice. The first part of the proof above shows that $Z_{0}=Z_{G}\left(\gamma_{0}\right)$ is discrete. Since $\gamma_{0} \in Z_{0}$, it follows that $Z_{0}$ is a lattice, so $\left[Z_{0}:\left\langle\gamma_{0}\right\rangle\right]$ is finite. Since $Z \leq Z_{0}$ (clearly) and $Z$ is infinite, $Z$ must have finite index in the virtually cyclic group $Z_{0}$. Thus $Z$ is a lattice, commensurable with $\Gamma$, and the proof is complete.

(6.2) Proposition (“Geometric Rigidity"). Let $\Gamma \in \operatorname{Lat}_{u}(X)$ and $\Gamma^{\prime} \in \operatorname{Lat}_{u}\left(X^{\prime}\right)$, where $X^{\prime}$ is another uniform tree. Let

$$
L\left(\Gamma, \Gamma^{\prime}\right)=\left\{h \in \operatorname{Hom}\left(\Gamma, \Gamma^{\prime}\right) \mid \exists \text { an h-equivariant graph morphism } X \rightarrow X^{\prime}\right\} .
$$

Then $\Gamma^{\prime} \backslash L\left(\Gamma, \Gamma^{\prime}\right)$ is finite, where $\Gamma^{\prime}$ acts by conjugation.

Proof. Form $\mathbf{A}=\Gamma \backslash \backslash X$ and $\mathbf{A}^{\prime}=\Gamma^{\prime} \backslash \backslash X^{\prime}$. Every $h \in L\left(\Gamma, \Gamma^{\prime}\right)$ arises from a morphism $\mathbf{A} \rightarrow \mathbf{A}^{\prime}$ via identification of $\Gamma$ and $\Gamma^{\prime}$ with fundamental groups of $\mathbf{A}$ and $\mathbf{A}^{\prime}$, respectively [B, I, (4.4)]. According to [B, I, (2.10)], there are only finitely many such homomorphisms modulo conjugation by $\Gamma^{\prime}$.

Remark. The proof does not require $\Gamma$ to be discrete, but only that $\Gamma \backslash X$ be finite and that each $\Gamma_{x}$ be finitely generated.

(6.3) Corollary. Let $\Gamma, \Gamma^{\prime} \in \operatorname{Lat}_{u}(X)$ and $N_{G}\left(\Gamma, \Gamma^{\prime}\right)=\left\{g \in G \mid g \Gamma g^{-1} \leq \Gamma^{\prime}\right\}$. Then $\Gamma^{\prime} \backslash N_{G}\left(\Gamma, \Gamma^{\prime}\right)$ is finite.

Proof. Define $\alpha: N_{G}\left(\Gamma, \Gamma^{\prime}\right) \rightarrow L_{G}\left(\Gamma, \Gamma^{\prime}\right)$ by $\alpha(g)=\operatorname{ad}(g) \mid \Gamma$; then $\alpha(g): \Gamma \rightarrow$ $\Gamma^{\prime}$ is a homomorphism such that $g: X \rightarrow X$ is $\alpha(g)$-equivariant. Moreover, $\alpha$ is $\Gamma^{\prime}$-equivariant, and factors to give an injective $\Gamma^{\prime}$-equivariant map

$$
N_{G}\left(\Gamma, \Gamma^{\prime}\right) / Z_{G}(\Gamma) \rightarrow L_{G}\left(\Gamma, \Gamma^{\prime}\right),
$$

hence an injection

$$
\Gamma^{\prime} \backslash N_{G}\left(\Gamma, \Gamma^{\prime}\right) / Z_{G}(\Gamma) \rightarrow \Gamma^{\prime} \backslash L_{G}\left(\Gamma, \Gamma^{\prime}\right) .
$$

The latter quotient is finite by $(6.2)$. If $Z_{G}(\Gamma)$ is finite, we thus conclude that $\Gamma^{\prime} \backslash N_{G}\left(\Gamma, \Gamma^{\prime}\right)$ is finite.

If $Z=Z_{G}(\Gamma)$ is not finite then, by (6.1), $X$ is virtually linear, and $\Gamma$ contains a cyclic group $\langle\gamma\rangle$ of finite index. Since $N_{G}\left(\Gamma, \Gamma^{\prime}\right) \subset N_{G}\left(\langle\gamma\rangle, \Gamma^{\prime}\right)$, we 
can, without loss, assume that $\Gamma=\langle\gamma\rangle$. If $h \in N_{G}\left(\Gamma, \Gamma^{\prime}\right)$ and $\Gamma_{h}=h \Gamma h^{-1} \leq$ $\Gamma^{\prime}$, then, by (5.6),

$$
\left[\Gamma^{\prime}: \Gamma_{h}\right]=\operatorname{Vol}(\Gamma \backslash \backslash X) / \operatorname{Vol}\left(\Gamma^{\prime} \backslash \backslash X\right)=v
$$

is independent of $h$. The finitely generated group $\Gamma$ has only finitely many subgroups of index $v$. Thus, fixing $h$ as above, we want the finiteness $\bmod \Gamma$ of the set of $g \in N_{G}\left(\Gamma, \Gamma^{\prime}\right)$ such that $g \Gamma g^{-1}=\Gamma_{h}$. Any such $g=n h$, where $n \in N_{h}=N_{G}\left(\Gamma_{h}\right)$. Since $\Gamma_{h}$ is cyclic, $N_{h}$ contains $Z_{h}=Z_{G}\left(\Gamma_{h}\right)$ with index $\leq 2$. By (6.1), $\left[Z_{h}: \Gamma_{h}\right]$ is finite. Hence, from $N_{h} \geq \Gamma_{h} \leq \Gamma^{\prime}$, we see that $N_{h}$ and $\Gamma^{\prime}$ are commensurable, and so $\Gamma^{\prime} \backslash \Gamma^{\prime} \cdot N_{h} \cong\left(\Gamma^{\prime} \cap N_{h}\right) \backslash N_{h}$ is finite, as required.

(6.4) Corollary. If $\Gamma \in \operatorname{Lat}_{u}(X)$, then $N_{G}(\Gamma) / \Gamma$ is finite.

Here, of course, $N_{G}(\Gamma)=N_{G}(\Gamma, \Gamma)$, because if $g \Gamma g^{-1} \leq \Gamma$ then $\operatorname{Vol}(\Gamma \backslash \backslash X)$ and $\left[\Gamma: g \Gamma g^{-1}\right] \operatorname{Vol}(\Gamma \backslash \backslash X)$ both equal $\operatorname{Vol}\left(g \Gamma g^{-1} \backslash \backslash X\right)$, so $g \Gamma g^{-1}=\Gamma$.

(6.5) Theorem. Let $\Gamma \in \operatorname{Lat}_{u}(X)$ and $m>0$. Then

$$
U^{m}(\Gamma)=\left\{\Gamma^{\prime} \leq G \mid \Gamma \leq \Gamma^{\prime} \text { and }\left[\Gamma^{\prime}: \Gamma\right] \leq m\right\}
$$

is finite.

Proof. To see this let $S$ denote the set of subgrours of $\Gamma$ of index dividing $m$ !. Since $\Gamma$ is finitely generated, $S$ is finite. We have a map $p: U^{m}(\Gamma) \rightarrow S$ defined by

$$
p\left(\Gamma^{\prime}\right)=\bigcap_{g \in \Gamma^{\prime}} g \Gamma g^{-1}=\operatorname{Ker}\left(\Gamma^{\prime} \rightarrow \operatorname{Aut}\left(\Gamma^{\prime} / \Gamma\right)\right),
$$

and $p\left(\Gamma^{\prime}\right) \triangleleft \Gamma^{\prime}$. If $p\left(\Gamma^{\prime}\right)=M \in S$, then $M \leq \Gamma^{\prime} \leq N_{G}(M)$, and $N_{G}(M) / M$ is finite by hypothesis. It follows that the fibers $p^{-1}(M)$ of $p$ are finite. We saw above that $S$ is finite; hence $U^{m}(\Gamma)$ is finite as claimed.

(6.6) Corollary. Let $\Gamma \leq G$ be discrete and $m>0$. Let $\Gamma_{0}$ be a finitely generated infinite subgroup of $\Gamma$. Then

$$
U_{\Gamma}^{m}\left(\Gamma_{0}\right)=\left\{\Gamma^{\prime} \leq \Gamma \mid \Gamma_{0} \leq \Gamma^{\prime} \text { and }\left[\Gamma^{\prime}: \Gamma_{0}\right] \leq m\right\}
$$

is finite.

Proof. The hypotheses on $\Gamma_{0}$ imply that $l\left(\Gamma_{0}\right) \neq 0$, where $l$ is the hyperbolic length function (cf. [B, (7.1) and (7.3)]). Let $Y=X_{\Gamma_{0}}$, the minimal $\Gamma_{0}$-invariant subtree [B, (7.5)]. If $\Gamma^{\prime} \in U_{\Gamma}^{m}\left(\Gamma_{0}\right)$, then $X_{\Gamma^{\prime}}=Y$ also [B, (7.7)]. Hence, $\Gamma^{\prime} \leq \Gamma_{Y}:=\{g \in \Gamma \mid g Y=Y\}$. Since $\Gamma_{Y}$ is discrete, the restriction homomorphism $r: \Gamma_{Y} \rightarrow \operatorname{Aut}(Y)$ has finite kernel and discrete image $r\left(\Gamma_{Y}\right)$. Since $\Gamma_{0}$ is finitely generated, it follows from [B, (7.9)(b)] that $\Gamma_{0} \backslash Y$ is finite. Hence, $r\left(\Gamma_{0}\right) \leq r\left(\Gamma_{Y}\right)$ are uniform $Y$-lattices, so $\left[r\left(\Gamma_{Y}\right): r\left(\Gamma_{0}\right)\right]$ is finite. Since $\operatorname{Ker}(r)$ is finite, it follows that $\left[\Gamma_{Y}: \Gamma_{0}\right]$ is finite. Finally,

$$
U_{\Gamma}^{m}\left(\Gamma_{0}\right) \subset\left\{\Gamma^{\prime} \mid \Gamma_{0} \leq \Gamma^{\prime} \leq \Gamma_{Y}\right\}
$$

is also finite. 
(6.7) Proposition. Let $\Gamma \in \operatorname{Lat}_{u}(X)$. Let $\Gamma_{0}, \Gamma_{1} \leq \Gamma$ be finitely generated subgroups. Then

$$
I_{\Gamma}\left(\Gamma_{0}, \Gamma_{1}\right)=\left\{\alpha \Gamma_{0} \alpha^{-1} \cap \beta \Gamma_{1} \beta^{-1} \mid \alpha, \beta \in \Gamma\right\}
$$

forms finitely many $\Gamma$-conjugacy classes.

Proof. Since $\Gamma$ has only finitely many conjugacy classes of finite subgroups (cf. $[B,(8.3)])$ it suffices to consider those intersections which are infinite. Further, modulo $\Gamma$-conjugation, we can restrict to intersections of the form $\Gamma_{\beta}=\Gamma_{0} \cap$ $\beta \Gamma_{1} \beta^{-1} \quad(\beta \in \Gamma)$.

Case 1. $\Gamma$ is free Then the proposition is a result of Imrich [Im] (cf. also Stallings [St, (5.7)(b)]).

Suppose that $\Gamma_{0}^{\prime} \leq \Gamma_{0}$ has finite index $m$. Define $\psi: I_{\Gamma}\left(\Gamma_{0}, \Gamma_{1}\right) \rightarrow I_{\Gamma}\left(\Gamma_{0}^{\prime}, \Gamma_{1}\right)$ $\Gamma$-equivariantly by $\psi\left(\Gamma_{\beta}\right)=\Gamma_{\beta}^{\prime}:=\Gamma_{0}^{\prime} \cap \Gamma_{\beta}$. Then $\left[\Gamma_{\beta}: \Gamma_{\beta}^{\prime}\right] \leq m$ so $\Gamma_{\beta} \in$ $U_{\Gamma}^{m}\left(\Gamma_{\beta}^{\prime}\right)$ (notation of $(6.6)$ ); thus $(6.6)$ implies that $\psi^{-1}\left(\Gamma_{\beta}^{\prime}\right)$ is finite when $\Gamma_{\beta}^{\prime}$ is infinite. Thus:

$$
\text { If } I_{\Gamma}\left(\Gamma_{0}^{\prime}, \Gamma_{1}\right) \text { is finite } \bmod \Gamma \text { then so also is } I_{\Gamma}\left(\Gamma_{0}, \Gamma_{1}\right) \text {. }
$$

Now let $\Gamma^{\prime} \triangleleft \Gamma$ be a free normal subgroup of finite index and put $\Gamma_{i}^{\prime}=\Gamma^{\prime} \cap \Gamma_{i}$ $(i=0,1)$. Let $A \subset \Gamma$ be a set of representatives of $\Gamma / \Gamma^{\prime}$. For $\alpha \in A$, Case 1 implies that $I_{\Gamma^{\prime}}\left(\alpha \Gamma_{0}^{\prime} \alpha^{-1}, \Gamma_{1}^{\prime}\right)$ is finite $\bmod \Gamma^{\prime}$-conjugation, say with representatives $\alpha \Gamma_{0}^{\prime} \alpha^{-1} \cap \beta \Gamma_{1}^{\prime} \beta^{-1}\left(\beta \in B_{\alpha} \subset \Gamma^{\prime}, B_{\alpha}\right.$ a finite set). If $\gamma \in \Gamma$, write $\gamma=\alpha^{-1} \gamma^{\prime}$ with $\alpha \in A$ and $\gamma^{\prime} \in \Gamma^{\prime}$. Then

$$
\Gamma_{0}^{\prime} \cap \gamma \Gamma_{1}^{\prime} \gamma^{-1}=\alpha^{-1}\left(\alpha \Gamma_{0}^{\prime} \alpha^{-1} \cap \gamma^{\prime} \Gamma_{1}^{\prime} \gamma^{\prime-1}\right) \alpha
$$

is $\Gamma$-conjugate to $\alpha \Gamma_{0}^{\prime} \alpha^{-1} \cap \beta \Gamma_{1}^{\prime} \beta^{-1}$ for some $\beta \in B_{\alpha}$. This shows that $I_{\Gamma}\left(\Gamma_{0}^{\prime}, \Gamma_{1}^{\prime}\right)$ is finite mod $\Gamma$-conjugation. Now applying $(*)$ above twice we conclude that $I_{\Gamma}\left(\Gamma_{0}, \Gamma_{1}\right)$ is finite and $\bmod \Gamma$-conjugation.

(6.8) Theorem. Let $H \leq G, v_{0}>0$, and $m_{0}>0$. Put

$$
\operatorname{Lat}_{u}^{v_{0}, m_{0}}(H)=\left\{\Gamma \in \operatorname{Lat}_{u}(H) \mid \operatorname{Vol}(\Gamma \backslash \backslash X) \leq v_{0} \text { and } m(\Gamma) \leq m_{0}\right\},
$$

where $(c f .(5.16)) m(\Gamma)=1 \mathrm{~cm}|F|, F$ varying over all finite subgroups of $\Gamma$. Then $\mathrm{Lat}_{u}^{v_{0}, m_{0}}(H)$ lies in finitely many $G_{H}$-conjugacy classes.

Proof. After subdividing $X$ if necessary we may assure that $H$ acts without inversion on $X$. We may clearly also assume that $\operatorname{Lat}_{u}(H) \neq \varnothing$. Choose $\Phi^{0} \leq G_{H}$ as in (4.7) so that $\Phi^{0} \backslash X=H \backslash X$. Let $\Gamma \in \mathrm{Lat}_{u}^{v_{0}, m_{0}}(H)$. By (2.8) $\Gamma$ contains a free subgroup $\Gamma^{0}$ of index $m(\Gamma)\left(\leq m_{0}\right)$. After $G_{H^{-}}$-conjugation (cf. (4.2)) we may assume that $\Gamma^{0} \leq \Phi^{0}$. Then

$$
\left[\Phi^{0}: \Gamma^{0}\right] \operatorname{Vol}\left(\Phi^{0} \backslash \backslash X\right)=\operatorname{Vol}\left(\Gamma^{0} \backslash \backslash X\right)=m(\Gamma) \operatorname{Vol}(\Gamma \backslash \backslash X) \leq m_{0} v_{0}
$$


so $\left[\Phi^{0}: \Gamma^{0}\right]$ is bounded by $m_{0} v_{0} \operatorname{Vol}\left(\Phi^{0} \backslash \backslash X\right)^{-1}$. The finitely generated group $\Phi^{0}$ contains only finitely many subgroups of a given index, whence only finitely many possibilities for $\Gamma^{0}$. For each given $\Gamma^{0}$ we have $\Gamma \in U^{m_{0}}\left(\Gamma^{0}\right)$, and the latter is finite by $(6.5)$.

(6.9) Corollary. If $X$ is not virtually linear, then, for $H \leq G, x_{0}>0, m_{0}>0$,

$$
\left\{\Gamma \in \operatorname{Lat}_{u}(H) \mid m(\Gamma) \leq m_{0} \text { and }|\chi(\Gamma)| \leq x_{0}\right\}
$$

lies in finitely many $G_{H}$-conjugacy classes.

Proof. There is a constant $\kappa(X)$ such that $\chi(\Gamma)=\kappa(X) \operatorname{Vol}(\Gamma \backslash \backslash X)$ for all $\Gamma \in \operatorname{Lat}_{u}(X)$ (cf. (5.8)). For $X$ not virtually linear, $\kappa(X) \neq 0$, so bounding $|\chi(\Gamma)|$ is equivalent to bounding $\operatorname{Vol}(\Gamma \backslash \backslash X)$, and then (6.9) follows from (6.8).

Remark. When $m_{0}=1$, the condition $m(\Gamma) \leq m_{0}$ above is equivalent to " $\Gamma$ is free." We shall see in $\S 7$ that, in (6.8) and (6.9), one cannot drop the bound on $m(\Gamma)$ without losing finiteness.

(6.10) Notation. Let $H \leq G$. For any group $\Gamma$ put

$\operatorname{Lat}_{u}(\Gamma, H)=\left\{h \in \operatorname{Hom}(\Gamma, H) \mid h\right.$ is injective and $\left.h(\Gamma) \in \operatorname{Lat}_{u}(H)\right\}$.

There is a natural action (right composition) of $\operatorname{Aut}(\Gamma)$ on $\operatorname{Lat}_{u}(\Gamma, H)$.

(6.11) Corollary ("Weak Rigidity"). Assume that $X$ is not virtually linear. Then $G_{H} \backslash \operatorname{Lat}_{u}(\Gamma, H) / \operatorname{Aut}(\Gamma)$ is finite, in the following sense. There exist elements $h_{1}, \ldots, h_{n} \in \operatorname{Lat}_{u}(\Gamma, H)$ such that any $h \in \operatorname{Lat}_{u}(\Gamma, H)$ is of the form $h=$ $\operatorname{ad}(g) \circ h_{i} \circ \alpha$ for some $i, \alpha \in \operatorname{Aut}(\Gamma)$, and $g \in G_{H}$.

Proof. The $\operatorname{Aut}(\Gamma)$-orbits on $\operatorname{Lat}_{u}(\Gamma, H)$ are just the sets of homomorphisms $h$ with a common image, $h(\Gamma)$. For any $h \in \operatorname{Lat}_{u}(\Gamma, H), m(h(\Gamma))=m(\Gamma)$ and $\chi(h(\Gamma))=\chi(\Gamma)$. Thus, by (6.9) the possible images $h(\Gamma)$ are finite modulo $G_{H}$-conjugacy.

(6.12). Consider next the case $H=G$. Then from (6.8),

$$
\operatorname{Lat}_{u}^{n_{0}, m_{0}}(X)=\left\{\Gamma \in \operatorname{Lat}_{u}(X) \mid \operatorname{Vol}(\Gamma \backslash \backslash X) \leq v_{0} \text { and } m(\Gamma) \leq m_{0}\right\}
$$

forms finitely many $G$-conjugacy classes. Moreover, putting, for a fixed group $\Gamma$,

$$
\operatorname{Lat}_{u}(\Gamma, X)=\left\{h \in \operatorname{Hom}(\Gamma, G) \mid h \text { is injective and } h(\Gamma) \in \operatorname{Lat}_{u}(X)\right\},
$$

we see that, when $X$ is not virtually linear,

$$
G \backslash \operatorname{Lat}_{u}(\Gamma, X) / \operatorname{Aut}(\Gamma)
$$

is finite. Suppose here that $\Gamma$ is a free group of rank $r$. For $h \in \operatorname{Lat}_{u}(\Gamma, X)$ put $A_{h}=h(\Gamma) \backslash X$, a finite graph covered by $X$. We have

$$
1-r=\chi(\Gamma)=\kappa(X) \operatorname{Vol}(\Gamma \backslash \backslash X)=\kappa(X) \cdot\left|A_{h}\right|
$$

SO

$$
n:=\frac{1-r}{\kappa(X)}=\left|A_{h}\right|
$$


is independent of $h$. If $\alpha \in \operatorname{Aut}(\Gamma)$ and $g \in G$, then $A_{h}=A_{h \circ \alpha}$ and $A_{g h g^{-1}} \cong$ $A_{h}$. Thus, we have a map $(h) \rightarrow\left(A_{h}\right)$ from $G \backslash \operatorname{Lat}_{u}(\Gamma, X) / \operatorname{Aut}(\Gamma)$ to the set $\operatorname{Graph}_{n}(X)$ of isomorphism classes $(A)$ of graphs $A$ with $n$ vertices covered by $X$. We claim that this map is bijective. In fact, let $(A) \in \operatorname{Graph}_{n}(X)$. Then we can write $A \cong \Gamma^{\prime} \backslash X$, where $\Gamma^{\prime} \cong \pi_{1}(A)$, and

$$
1-\operatorname{rank}\left(\Gamma^{\prime}\right)=\kappa(X) \cdot|A|=\kappa(X) \cdot n,
$$

so $\operatorname{rank}\left(\Gamma^{\prime}\right)=r$. Hence, $\Gamma^{\prime}=h(\Gamma)$ for some $h \in \operatorname{Lat}_{u}(\Gamma, X)$ and so $A \cong$ $A_{h}$. Finally, suppose that $h, h^{\prime} \in \operatorname{Lat}_{u}(\Gamma, X)$ and there is an isomorphism $\psi: A_{h} \rightarrow A_{h}^{\prime}$. Choose $a_{0} \in A_{h}$ and put $a_{0}^{\prime}=\psi\left(a_{0}\right)$. Identifying $X$ with $\left(\widetilde{A_{h}, a_{0}}\right)$ and $\left(\widetilde{A_{h^{\prime}}, a_{0}^{\prime}}\right)$, we obtain an isomorphism $g: X \rightarrow X$ covering $\psi$ so that $g h(\Gamma) g^{-1}=h^{\prime}(\Gamma)$. It follows that $h^{\prime}=\operatorname{ad}(g) \circ h \circ \alpha$ for some $\alpha \in \operatorname{Aut}(\Gamma)$.

We can formulate our conclusion as follows.

(6.13) Proposition. Let $X$ be a uniform tree which is not virtually linear. Let $\Gamma_{r}$ be a free group of rank $r$, and put $n=(1-r) / \kappa(X)$. Let

$\operatorname{Lat}_{u}\left(\Gamma_{r}, X\right)=\left\{h \in \operatorname{Hom}\left(\Gamma_{r}, G\right) \mid h\right.$ is injective and $\left.h\left(\Gamma_{r}\right) \in \operatorname{Lat}_{u}(X)\right\}$, where $G=\operatorname{Aut}(X)$. The map $h \mapsto A_{h}=h\left(\Gamma_{r}\right) \backslash X$ induces a bijection from $G \backslash \operatorname{Lat}_{u}\left(\Gamma_{r}, X\right) / \operatorname{Aut}\left(\Gamma_{r}\right)$ to the set of isomorphism classes of graphs with $n$ vertices covered by $X$. (Of course these sets are empty if $n \notin \mathbf{Z}$.)

(6.14) Examples. 1. For the homogeneous tree $X_{k}(k \geq 3)$ we have $\kappa\left(X_{k}\right)=$ $1-k / 2$ (cf. (5.13)) and so $n=(1-r)(2 /(2-k))=2(r-1) /(k-2)$, and $r=$ $1+n(k-2) / 2$. Thus the $n$ vertex connected $k$-regular graphs are parametrized by $G \backslash \operatorname{Lat}\left(\Gamma_{r}, X\right) / \operatorname{Aut}\left(\Gamma_{r}\right)$, where $\Gamma_{r}$ is free of rank $r=1+n(k-1) / 2$. (When $r \notin \mathbf{Z}$ there are no such graphs.)

2. Let $X=X_{p, q}$, the bihomogeneous bipartite tree with indices $p, q$. We have $\kappa(X)=(p+q-p q) /(p+q)$ (cf. (5.13)) and consequently $n=$ $(1-r)(p+q) /(p+q-p q)$, whence $r=1+n(p q-p-q) /(p+q)$. Thus $G \backslash \operatorname{Lat}_{u}\left(\Gamma_{r}, X_{p, q}\right) / \operatorname{Aut}\left(\Gamma_{r}\right)$ parametrizes $(p, q)$-biregular connected bipartite graphs.

\section{APPENDIX B: COMMENSURATORS}

(B.1) Abstract commensurators. We discuss here a notion pointed out to us by J.-P. Serre and by Walter Neumann. Let $\Gamma$ be a group. Let $\mathrm{FI}(\Gamma)$ denote the set of subgroups of finite index in $\Gamma$. Let $U=U(\Gamma)$ denote the set of pairs $\left(\alpha, \Gamma^{\prime}\right)$, where $\Gamma^{\prime} \in \mathrm{FI}(\Gamma)$ and $\alpha: \Gamma^{\prime} \rightarrow \Gamma$ is a monomorphism with $\alpha \Gamma^{\prime} \in \operatorname{FI}(\Gamma)$. Define an equivalence relation on $U$ by $\left(\alpha_{1}, \Gamma_{1}\right) \sim\left(\alpha_{2}, \Gamma_{2}\right)$ if $\alpha_{1}\left|\Gamma_{3}=\alpha_{2}\right| \Gamma_{3}$ for some $\Gamma_{3} \in \operatorname{FI}\left(\Gamma_{1} \cap \Gamma_{2}\right)$. Put $\left[\alpha, \Gamma^{\prime}\right]$ (or $\left.[\alpha]\right)=$ the $\sim$-class of $\left(\alpha, \Gamma^{\prime}\right)$ and

$$
C(\Gamma)=\left\{\left[\alpha, \Gamma^{\prime}\right] \mid(\alpha, \Gamma) \in U\right\}=U / \sim .
$$

We can make $C(\Gamma)$ into a group, with composition

$$
\left[\alpha, \Gamma_{\alpha}\right] \circ\left[\beta, \Gamma_{\beta}\right]=\left[\gamma, \Gamma_{\gamma}\right],
$$


where $\Gamma_{\gamma}=\beta^{-1}\left(\beta \Gamma_{\beta} \cap \Gamma_{\alpha}\right)$ and $\gamma=\alpha \circ \beta$ on $\Gamma_{\gamma}$. The inverse is $\left[\alpha, \Gamma_{\alpha}\right]^{-1}=$ $\left[\alpha^{-1}, \alpha \Gamma_{\alpha}\right]$. This group $C(\Gamma)$ is called the (abstract) commensurator of $\Gamma$.

(B.2) Examples. 1. $C\left(\mathbf{Z}^{n}\right)=G L_{n}(\mathbf{Q}) \quad\left(=\operatorname{Aut}\left(\mathbf{Q}^{n}\right)\right)$.

2. For $n \geq 3, C\left(S L_{n}(\mathbf{Z})\right)=P G L_{n}(\mathbf{Q}) \rtimes\langle\sigma\rangle$, where $\sigma=$ transpose inverse. This uses rigidity properties of $S L_{n}(\mathrm{Z})$ (cf. [BMS]).

3. If $\Gamma^{\prime} \in \mathrm{FI}(\Gamma)$, then evidently $C\left(\Gamma^{\prime}\right)=C(\Gamma)$. Hence $C\left(S L_{2}(\mathrm{Z})\right)=C\left(F_{n}\right)$ for all $n \geq 2$, where $F_{n}$ denotes the free group on $n$ generators.

Problem. What interesting things can be said about this group?

(B.3). There is an evident homomorphism $\phi: \operatorname{Aut}(\Gamma) \rightarrow C(\Gamma)$, and $\phi(\alpha)=1$ iff the fixed points of $\alpha$ form a subgroup of finite index. The composite

$$
\Gamma \stackrel{\text { add }}{\rightarrow} \operatorname{Aut}(\Gamma) \stackrel{\phi}{\rightarrow} C(\Gamma)
$$

has kernel $\bigcup_{\Gamma^{\prime} \in \mathrm{FI}(\Gamma)} Z_{\Gamma}\left(\Gamma^{\prime}\right)$.

Example. If $\Gamma$ is an infinite group with no proper subgroups of finite index (e.g., an infinite simple group), then $\phi$ is evidently an isomorphism.

(B.4). Suppose that $\Gamma$ is a subgroup of a group $G$. The commensurator of $\Gamma$ in $G$ is

$$
C_{G}(\Gamma)=\left\{g \in G \mid g \Gamma g^{-1} \text { and } \Gamma \text { are commensurable }\right\} .
$$

Then

is a homomorphism, and

$$
\begin{aligned}
\phi_{G}: C_{G}(\Gamma) & \rightarrow C(\Gamma) \\
g & \mapsto\left[\operatorname{ad}(g), \Gamma \cap g^{-1} \Gamma g\right]
\end{aligned}
$$

$$
\operatorname{Ker}\left(\phi_{G}\right)=\bigcup_{\Gamma^{\prime} \in \mathrm{F}(\Gamma)} Z_{G}\left(\Gamma^{\prime}\right) .
$$

(B.5) Proposition. If $\Gamma$ is finitely generated, then $C(\Gamma)$ is countable, as is $\mathrm{FI}(\Gamma)$. Proof. For $\Gamma^{\prime} \in \mathrm{FI}(\Gamma)$ put

$$
\operatorname{Mon}^{c}\left(\Gamma^{\prime}, \Gamma\right)=\left\{\alpha \mid\left(\alpha, \Gamma^{\prime}\right) \in U\right\} \subset \operatorname{Hom}\left(\Gamma^{\prime}, \Gamma\right) .
$$

Then

$$
C(\Gamma)=\underset{\Gamma^{\prime} \in \mathrm{FI}(\Gamma)}{\lim } \operatorname{Mon}^{c}\left(\Gamma^{\prime}, \Gamma\right),
$$

where the maps in the inductive system are restrictions to smaller subgroups. Since $\Gamma$ is finitely generated, there are only finitely many $\Gamma^{\prime} \leq \Gamma$ of a given finite index; hence $\mathrm{FI}(\Gamma)$ is countable. If $\Gamma^{\prime} \in \mathrm{FI}(\Gamma)$, then $\Gamma^{\prime}$ is finitely generated, so $\operatorname{Hom}\left(\Gamma^{\prime}, \Gamma\right)$ is countable, and hence so also is $\operatorname{Mon}^{c}\left(\Gamma^{\prime}, \Gamma\right)$. Whence the proposition.

(8.6) Corollary. If $X$ is a uniform tree, $G=\operatorname{Aut}(X)$, and $\Gamma \in \operatorname{Lat}_{u}(X)$, then $C_{G}(\Gamma)$ is countable.

Proof. If $X$ is finite, $G$ is finite. If $X$ is infinite we have

$$
\phi_{G}: C_{G}(\Gamma) \rightarrow C(\Gamma)
$$


with $C(\Gamma)$ countable, by (B.5). Moreover $\operatorname{Ker}\left(\phi_{G}\right)$ is the union of $Z_{G}\left(\Gamma^{\prime}\right)$ $\left(\Gamma^{\prime} \in \mathrm{FI}(\Gamma)\right)$, and $\mathrm{FI}(\Gamma)$ is countable (B.5), so it suffices to see that each $Z_{G}\left(\Gamma^{\prime}\right)$ is countable. The latter follows from (6.1).

(B.7) The commensurator of a uniform tree lattice. Let $X$ be a uniform tree $((4.11))$ and $G=\operatorname{Aut}(X)$. Assume that $X$ is hyperbolic ((5.11)), i.e., infinite and not virtually linear. Assume further that $X$ is minimal, i.e., that $G$ leaves no proper subtree invariant.

Let $\Gamma \in \operatorname{Lat}_{u}(X)$. Then it follows from (B.4) and (6.1) that

$$
C_{G}(\Gamma) \rightarrow C(\Gamma) \text { is injective. }
$$

Walter Neumann pointed out to us that this cannot be surjective, because $N_{G}(\Gamma) / \Gamma$ is finite $((6.4))$ whereas $N_{C(\Gamma)}(\Gamma)=\operatorname{Aut}(\Gamma)$, with $\Gamma \leq \operatorname{Aut}(\Gamma) \leq C(\Gamma)$, again using (6.1). Taking $\Gamma$ a free lattice, for example, we see that $\operatorname{Out}(\Gamma)=$ $\operatorname{Aut}(\Gamma) / \Gamma$ is infinite.

(B.8) End stabilizers. Let $X$ be a tree and $\varepsilon$ an (open) end of $X$. If $x \in X$ let $[x, \varepsilon)$ denote the ray (infinite half-line) from $x$ to $\varepsilon$. For $y \in X$ define

$$
(x-y)_{\varepsilon}=d(y, z)-d(x, z)
$$

for any $z \in[x, \varepsilon) \cap[y, \varepsilon)$; this is independent of $z$ :

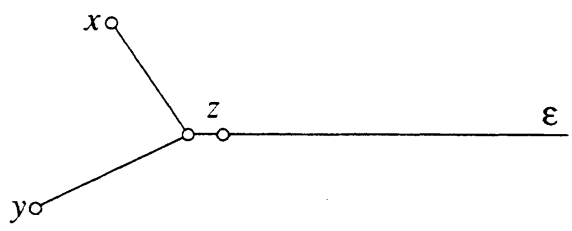

In $G=\operatorname{Aut}(X)$, the stabilizer $G_{\varepsilon}$ of $\varepsilon$ admits the homomorphism

$$
\begin{aligned}
\tau_{\varepsilon}: G_{\varepsilon} & \rightarrow \mathbf{Z}, \\
\tau_{\varepsilon}(\gamma) & =(\gamma x-x)_{\varepsilon} \quad \text { for any } x \in X
\end{aligned}
$$

(cf. $[B 2,(1.6)])$. We have

$$
l(\gamma)=\left|\tau_{\varepsilon}(\gamma)\right| \text { for } \gamma \in G_{\varepsilon}
$$

(loc. cit.). If $\gamma \in G_{\varepsilon}$ and $x \in X_{\gamma}$, then $[x, \varepsilon) \subset X_{\gamma}$.

(B.9) $\Gamma$-hyperbolic ends. Let $X$ be a uniform tree, $G=\operatorname{Aut}(X), \Gamma \in \operatorname{Lat}_{u}(X)$. Let $\varepsilon$ be an end of $X$ and consider the exact sequence

$$
1 \rightarrow \Gamma_{\varepsilon}^{0} \rightarrow \Gamma_{\varepsilon} \stackrel{\tau_{\varepsilon}}{\rightarrow} \mathbf{Z}
$$

Since $l\left(\Gamma_{\varepsilon}^{0}\right)=0$ and $\Gamma \in \operatorname{Lat}_{u}(X)$ has bounded torsion, it follows from $[\mathrm{B},(7.2)]$ that

$$
\Gamma_{\varepsilon}^{0} \text { is finite. }
$$

We call $\varepsilon$ a $\Gamma$-hyperbolic end of $X$ if $\tau_{\varepsilon}\left(\Gamma_{\varepsilon}\right) \neq 0$. 
If $\gamma \in \Gamma$ is hyperbolic we put:

$$
\varepsilon_{\gamma}=\text { the end of } X_{\gamma} \text { toward which } \gamma \text { translates. }
$$

We have $\varepsilon_{\gamma}=\varepsilon$ iff $\gamma \in \Gamma_{\varepsilon}$ and $\tau_{\varepsilon}(\gamma)>0$. In this case $\langle\gamma\rangle$ has finite index in $\Gamma_{\varepsilon}$, by (1). Consequently, if $\gamma^{\prime} \in \Gamma$ is hyperbolic and $\varepsilon_{\gamma^{\prime}}=\varepsilon_{\gamma}$, then $\gamma^{\prime n}=\gamma^{m}$ for some $n, m>0$. The converse is trivial. Thus:

$$
\begin{aligned}
& \text { If } \gamma, \gamma^{\prime} \in \Gamma \text { are hyperbolic, then } \\
& \varepsilon_{\gamma^{\prime}}=\varepsilon_{\gamma} \text { iff } \gamma^{\prime n}=\gamma^{m} \text { for some } n, m>0 .
\end{aligned}
$$

We shall write $\operatorname{HE}(\Gamma)=$ the set of $\Gamma$-hyperbolic ends of $X$. This is group theoretically definable as the equivalence classes of elements of infinite order in $\Gamma$ for the relation defined by (2).

If $\Gamma$ and $\Gamma^{\prime}$ are commensurable, then $\operatorname{HE}(\Gamma)=\operatorname{HE}\left(\Gamma^{\prime}\right)$. In fact, if $\gamma \in \Gamma$, then $\gamma^{n} \in \Gamma^{\prime}$ for some $n>0$, so $\operatorname{HE}(\Gamma) \subset \operatorname{HE}\left(\Gamma^{\prime}\right)$, whence $\operatorname{HE}(\Gamma)=\operatorname{HE}\left(\Gamma^{\prime}\right)$ by symmetry.

(B.10) The action of $C(\Gamma)$ on $\operatorname{HE}(\Gamma)$. As above, let $X$ be a uniform tree, $G=\operatorname{Aut}(X), \Gamma \in \operatorname{Lat}_{u}(X)$. Let $g \in C(\Gamma)$, say $g=\left[\alpha, \Gamma^{\prime}\right]$ with $\Gamma^{\prime} \in \operatorname{FI}(\Gamma)$. Then $\alpha$ defines a map

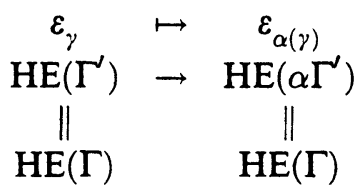

It is easily seen, using (B.9), that this is well defined, depends only on $g$, and defines an action $h: C(\Gamma) \rightarrow \operatorname{Aut}(\operatorname{HE}(\Gamma))$, where the latter refers to set automorphisms of $\operatorname{HE}(\Gamma)$.

If $X$ is finite, $C(\Gamma)=\{1\}$. If $X$ is virtually linear, then $C(\Gamma) \cong C(\mathbf{Z})=$ $\mathbf{Q}^{\times}, \operatorname{HE}(\Gamma)$ has two elements, and $h$ is surjective with kernel the positive rationals.

(B.11) Proposition. If $X$ is hyperbolic, then $h: C(\Gamma) \rightarrow \operatorname{Aut}(\operatorname{HE}(\Gamma))$ is injective. Proof. We are at liberty to replace $\Gamma$ by a subgroup of finite index, and so assume that $\Gamma$ is free. Call $\gamma$ in $\Gamma$ primitive in $\Gamma$ if $\gamma$ generates its own centralizer $Z_{\Gamma}(\gamma)$. For any $\gamma \neq 1$ in $\Gamma$, there is a unique primitive element $\sqrt{\gamma}$ (generating $Z_{\Gamma}(\gamma)$ ) such that $\gamma=\sqrt{\gamma}^{n}$ with $n$ an integer $>0$.

Now let $\left[\alpha, \Gamma^{\prime}\right] \in \operatorname{Ker}(h)$. Then for $\gamma \neq 1$ in $\Gamma^{\prime}$ we have $\varepsilon_{\gamma}=\varepsilon_{\alpha(\gamma)}$, hence $\alpha(\gamma)^{n}=\gamma^{m}$ for some $n, m>0$, hence $\sqrt{\alpha(\gamma)}=\sqrt{\gamma}$ in $\Gamma$. Let $\gamma, \delta$ be distinct members of a basis of $\Gamma^{\prime}$. Such exist because $X$ is hyperbolic. Then, by (B.12) below, for all sufficiently large $m, \gamma \delta^{m}$ and $\alpha(\gamma) \alpha(\delta)^{m}$ are primitive in $\Gamma$, and so $\gamma \delta^{m}=\sqrt{\gamma \delta^{m}}=\sqrt{\alpha\left(\gamma \delta^{m}\right)}=\alpha(\gamma) \alpha(\delta)^{m}$. Using the analogous equation with $m+1$ in place of $m$ we conclude that $\alpha(\delta)=\delta$. Hence $\alpha$ fixed each basis element of $\Gamma^{\prime}$, so $\alpha=\mathrm{Id}_{\Gamma^{\prime}}$.

The following lemma was suggested to us by Geoffrey Mess. 
(B.12) Lemma. Let $\Gamma$ be a free group. Let $\gamma, \delta$ be a basis for a free subgroup. For all sufficiently large $m>0, \gamma \delta^{m}$ is primitive in $\Gamma$.

Proof. Fix a basis $B$ of $\Gamma$. For $\gamma \in \Gamma$ let $L(\gamma)$ denote the length of the reduced word in $B$ representing $\gamma$. Call a product $\gamma \cdot \delta$ in $\Gamma$ reduced if $L(\gamma \delta)=L(\gamma)+L(\delta)$.

Now let $\gamma, \delta$ be a basis for a free subgroup of $\Gamma$. After conjugation we can assume that $\delta$ is cyclically reduced, i.e., $L\left(\delta^{m}\right)=m L(\delta)$ for $m \geq 0$. Choose $m_{0}>0$ so that $m_{0} L(\delta)>L(\gamma)$. Then for $m \geq m_{0}$ we have

$$
L\left(\gamma \delta^{m}\right)=L\left(\gamma \delta^{m_{0}}\right)+\left(m-m_{0}\right) L(\varsigma:
$$

Suppose that $\left(m-m_{0}\right) L(\delta)>L\left(\gamma \delta^{m_{0}}\right)$. Let $\varepsilon=\sqrt{\gamma \delta^{m}}$ and write $\gamma \delta^{m}=\varepsilon^{n}$. We claim that, for $m$ sufficiently large, $n=1$. Suppose, on the contrary, that $n \geq 2$. Since the product $\gamma \delta^{m}=\left(\gamma \delta^{m_{0}}\right) \cdot \delta^{m-m_{0}}$ is reduced and the second factor is longer than the first, we must have

$$
\varepsilon=\delta_{1} \delta^{r}, \quad r \geq 0, \delta=\delta_{0} \cdot \delta_{1} \text { (reduced product), }
$$

and $\delta_{1} \neq 1$. From $\gamma \delta^{m}=\varepsilon^{n}=\left(\delta_{1} \delta^{n}\right)^{n}$ we obtain

$$
\gamma \delta^{m-r-1} \cdot \delta_{0}=\varepsilon^{n-1}
$$

Write $\delta_{1}=\alpha \cdot \delta_{1}^{\prime} \cdot \alpha^{-1}$ (reduced) with $\delta_{1}^{\prime}$ cyclically reduced. Then $\varepsilon=\delta_{1} \delta^{r}=$ $\alpha \cdot\left(\delta_{1}^{\prime} \cdot \alpha^{-1} \cdot \delta^{r-1} \cdot \delta_{0} \cdot \alpha \cdot \delta_{1}^{\prime}\right) \cdot \alpha^{-1}=\alpha \cdot \Delta \cdot \alpha^{-1}$ (reduced), with $\Delta$ cyclically reduced. Thus $\gamma \delta^{m-r-1} \cdot \delta_{0}=\alpha \cdot \Delta^{n-1} \cdot \alpha^{-1}$. If $\delta_{0} \neq 1$ it follows that $\delta_{0} \cdot \alpha$ is not reduced. This contradicts the fact that $\delta=\delta_{0} \cdot \delta_{1}=\delta_{0} \cdot \alpha \cdot \delta_{1}^{\prime} \cdot \alpha^{-1}$ is reduced. Thus $\delta_{0}=1$, i.e. $\varepsilon=\delta^{r+1}$, so $\gamma \delta^{m}=\left(\delta^{r+1}\right)^{n}$, whence $\gamma \in\langle\delta\rangle$, contrary to our hypothesis.

(B.13) The metric topology on $\operatorname{Ends}(X)$. Let $X$ be a tree and let $\operatorname{Ends}(X)$ denote the set of (open) ends of $X$. Fix a base point $x_{0} \in X$. For $\varepsilon, \varepsilon^{\prime} \in$ $\operatorname{Ends}(X)$ put

$$
\begin{gathered}
\varepsilon \cap \varepsilon^{\prime}=\left[x_{0}, \varepsilon\right) \cap\left[x_{0}, \varepsilon^{\prime}\right), \\
\left|\varepsilon \cap \varepsilon^{\prime}\right|=\text { length }\left(\varepsilon \cap \varepsilon^{\prime}\right) \quad(\leq \infty),
\end{gathered}
$$

and

$$
d\left(\varepsilon, \varepsilon^{\prime}\right)=\frac{1}{\left|\varepsilon \cap \varepsilon^{\prime}\right|+1} .
$$

We claim that this defines a metric (of diameter 1) on $\operatorname{Ends}(X)$. In fact, if $\varepsilon, \varepsilon^{\prime}, \varepsilon^{\prime \prime}$ are ends, then two of $\varepsilon \cap \varepsilon^{\prime}, \varepsilon \cap \varepsilon^{\prime \prime}$, and $\varepsilon^{\prime} \cap \varepsilon^{\prime \prime}$ are equal and contained in the third:

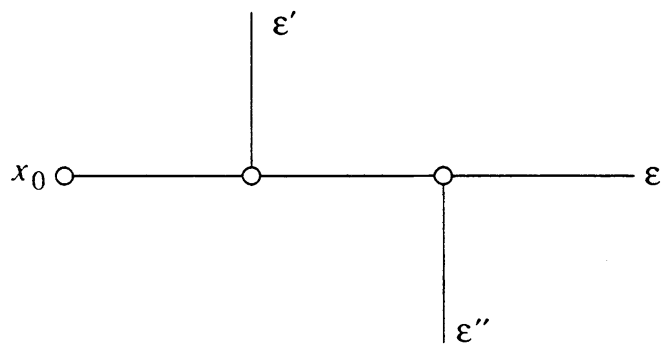


Hence, the two smaller of $\left|\varepsilon \cap \varepsilon^{\prime}\right|,\left|\varepsilon \cap \varepsilon^{\prime \prime}\right|$, and $\left|\varepsilon^{\prime} \cap \varepsilon^{\prime \prime}\right|$ are equal, so the two larger of $d\left(\varepsilon, \varepsilon^{\prime}\right), d\left(\varepsilon, \varepsilon^{\prime \prime}\right)$, and $d\left(\varepsilon^{\prime}, \varepsilon^{\prime \prime}\right)$ are equal; hence,

$$
d\left(\varepsilon, \varepsilon^{\prime \prime}\right) \leq \max \left(d\left(\varepsilon, \varepsilon^{\prime}\right), d\left(\varepsilon^{\prime}, \varepsilon^{\prime \prime}\right)\right) .
$$

It is easily seen that the topology defined by $d=d_{x_{0}}$ does not depend on the choice of $x_{0}$. If $g \in \operatorname{Aut}(X)$, then $g: \operatorname{Ends}(X) \rightarrow \operatorname{Ends}(X)$ is isometric from $d_{x_{0}}$ to $d_{g x_{0}}$, hence continuous.

(B.14) Questions. Let $X$ be a uniform tree, $G=\operatorname{Aut}(X)$, and $C(X)=C_{G}\left(\Gamma_{0}\right)$ for some $\Gamma_{0} \in \operatorname{Lat}_{u}(X)$.

I. Is the action of $C\left(\Gamma_{0}\right)$ on $\operatorname{HE}\left(\Gamma_{0}\right) \subset \operatorname{Ends}(X)$ (cf. (B.10)) continuous for the induced topology (cf. (B.13))?

II. If $\Gamma \in \operatorname{Lat}_{u}(X)$ and $C_{G}(\Gamma)=C_{G}\left(\Gamma_{0}\right)$, then must $\Gamma$ be commensurable with $\Gamma_{0}$ ?

III. If $\Gamma \leq C(X)$ is finitely generated, how can we (group theoretically in $C(X))$ recognize when $\Gamma$ has a fixed point in $X$ ? When $\Gamma \in \operatorname{Lat}_{u}(X)$ ?

IV. Does the group $C(X)$ (or $G$ ) determine $X$ up to isomorphism?

\section{NONFINITENESS PHENOMENA}

We shall here construct examples of uniform lattices which illustrate, for example, the following phenomena.

(7.1) Theorem. Let $X_{n}$ denote the $n$-homogeneous tree.

(a) For $n \geq 3$, Lat $_{u}\left(X_{n}\right)$ contains infinite ascending chains $\Gamma_{1}<\Gamma_{2}<\Gamma_{3}<$ ... In particular, $\operatorname{Vol}\left(\Gamma_{i} \backslash \backslash X_{n}\right) \rightarrow 0$ as $i \rightarrow \infty$.

(b) Suppose that $n \geq 5$. Given an integer $v>0, v$ even if $n=5$, there exist infinitely many conjugacy classes of $\Gamma \in \operatorname{Lat}_{u}\left(X_{n}\right)$ such that $\operatorname{Vol}\left(\Gamma \backslash \backslash X_{n}\right)=v$.

Assertion (b) responds to a question posed to us by A. Borel.

(7.2) $\mathbf{A}$ method for constructing lattices on $X$. Let $\mathbf{A}=(A, \mathscr{A})$ be a graph of groups, $a_{0} \in A, X=\left(\widetilde{\mathbf{A}, a_{0}}\right), \Gamma=\pi_{1}\left(\mathbf{A}, a_{0}\right)$, and $G=\operatorname{Aut}(X)$. Then $\Gamma$ acts on $X$, with quotient $X \stackrel{p}{\rightarrow} \Gamma \backslash X=A$, whence a homomorphism $\rho: \Gamma \rightarrow G$. The action is discrete iff $\mathscr{A}_{a}$ is finite for all $a \in A$ (since $\Gamma_{x} \cong \mathscr{A}_{p(x)}$ ), it is cocompact iff $A=\Gamma \backslash X$ is finite, and it is effective, i.e., $\rho: \Gamma \rightarrow G$ is injective, iff $A$ is effective in the sense of [B, I, (1.24)]. Thus:

$$
\Gamma=\pi_{1}\left(\mathbf{A}, a_{0}\right) \text { is a uniform lattice on } X=\left(\widetilde{\mathbf{A}, a_{0}}\right) \text { iff } \mathbf{A} \text { is an }
$$
effective finite graph of finite groups.

Further, from [B, I, (2.4), (2.7), and (3.6)] we have:

If $H \leq G$ acts without inversion on $X$, then constructing $g \in G$ so that $g \Gamma g^{-1} \leq H$ is equivalent to constructing a covering morphism $\mathbf{A} \rightarrow H \backslash \backslash X$.

(7.3) We shall construct $\mathbf{A}$ instead of $\Gamma$. In practice this is done in two steps.

I. Construct the underlying edge-indexed finite graph $(A, i)=I(\mathbf{A})$, where, 
recall, $\left.i(e)=\mid \mathscr{A}_{\partial_{0} e}: \alpha_{e} \mathscr{A}_{e}\right]$ for $e \in E(A)$. Observe that the tree $X=\left(\widetilde{\mathbf{A}, a_{0}}\right)=$ $\left(A, \widetilde{i}, a_{0}\right)$ is determined by $(A, i)$ up to isomorphism (cf. [B, $\left.\mathrm{I},(1.18)\right]$ ). For $a \in A$ put $i(a)=\sum_{\partial_{0} e=a} i(e)$. Then $X=X_{n}$, the $n$-homogeneous tree, iff $i(a)=n$ for all $a \in A$. Similarly, $X=X_{m, n}$, the bihomogeneous bipartite tree with indices $m, n$ iff $A$ is bipartite with $i(a)=m$, respectively $n$, in the components of the bipartition.

II. Given the finite edge-indexed graph $(A, i)$, we wish to find a finite grouping $\mathbf{A}=(A, \mathscr{A})$ of $(A, i)$ such that $\mathbf{A}$ is effective. According to (2.4), a finite grouping exists iff $(A, i)$ is unimodular. In an infinite family of such finite groupings $\mathbf{A}$ the sizes of the groups $\mathscr{A}_{a}$ must increase, whereas this largeness of the $\mathscr{A}_{a}$ makes it more difficult to make $\mathbf{A}$ effective. Resolving this issue becomes, in each case, an interesting problem in finite group theory (see, e.g., (7.16)).

(7.4) Example. Consider the loop $L$ with indices $m, m$ :

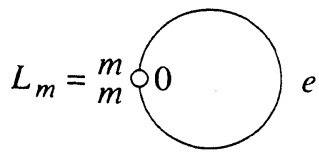

It has vertex 0 and indices $i(e)=m=i(\bar{e})$. (Equality of indices is required for unimodularity.) We have $\left(\widetilde{L_{m}}, 0\right)=X_{2 m}$, the $2 m$-homogeneous tree. Let $M$ be a group of order $m$. For each integer $r>0$ put $V_{r}=M^{(\mathbf{Z} / r \mathrm{Z})}$, the group of set functions $x: \mathbf{Z} / r \mathbf{Z} \rightarrow M$. Define $\alpha_{r} \in \operatorname{Aut}\left(V_{r}\right)$ by $\alpha_{r}(x)(i)=x(i+1)$. Let

$$
W_{r}=\left\{x \in V_{r} \mid x(0)=1 \in M\right\} \quad\left(\cong M^{r-1}\right) .
$$

Define $\mathbf{A}_{r}$ (or $\left.\mathbf{A}_{M, r}\right)=\left(L, \mathscr{A}^{(r)}\right.$ ) by $\mathscr{A}_{0}^{(r)}=V_{r}, \mathscr{A}_{e}^{(r)}=\mathscr{A}_{\bar{e}}^{(r)}=W_{r}, \alpha_{e}=$ $\alpha_{r} \mid W_{r}: W_{r} \rightarrow V_{r}$, and $\alpha_{\bar{e}}=$ inclusion. Then clearly $\mathbf{A}_{r}$ is a finite grouping of $L_{m}, \Gamma_{r}=\pi_{1}\left(\mathbf{A}_{r}, 0\right)=\left\langle V_{r}, e\right| e x e^{-1}=\alpha_{r}(x)$ for all $\left.x \in W_{r}\right\rangle, \Gamma_{r} \backslash X_{2 m}=L$, and $\operatorname{Vol}\left(\Gamma_{r} \backslash \backslash X_{2 m}\right)=1 / m^{r}$. Finally, we claim that $\mathbf{A}_{r}$ is effective. According to $[\mathrm{B}, \mathrm{I},(1.23)]$ we must show that if $N \leq W_{r}$ is normal in $V_{r}$ and $\alpha_{r}(N)=N$, then $N=\{1\}$. This follows from the obvious fact that $\bigcap_{i=0}^{r} \alpha_{r}^{i}\left(W_{r}\right)=\{1\}$.

(7.5). Suppose that $r$ divides $r^{\prime}$. Let $p: \mathbf{Z} / r^{\prime} \mathbf{Z} \rightarrow \mathbf{Z} / r \mathbf{Z}$ be the natural projection. This defines an embedding

$$
\begin{gathered}
\psi: V_{r}=M^{(\mathbf{Z} / r \mathbf{Z})} \rightarrow V_{r^{\prime}}=M^{\left(\mathbf{Z} / r^{\prime} \mathbf{Z}\right)}, \\
\psi(x)(i)=x(p(i)) \quad \text { for } x \in V_{r}, \quad i \in \mathbf{Z} / r^{\prime} \mathbf{Z} .
\end{gathered}
$$


Clearly $\psi\left(W_{r}\right) \leq W_{r^{\prime}}$. Moreover,

$$
\begin{aligned}
\psi\left(\alpha_{r}(x)\right)(i) & =\alpha_{r}(x)(p(i))=x(p(i)+1) \\
& =x(p(i+1))=\psi(x)(i+1)=\alpha_{r^{\prime}}(\psi(x))(i)
\end{aligned}
$$

i.e., $\psi \circ \alpha_{r}=\alpha_{r^{\prime}} \circ \psi$. Hence $\psi$ defines an inclusion of graphs of groups $\Phi: \mathbf{A}_{r} \rightarrow \mathbf{A}_{r^{\prime}}$. Since the $\psi$-induced maps on cosets $V_{r} / W_{r} \rightarrow V_{r^{\prime}} / W_{r^{\prime}}$ and $V_{r} / \alpha_{r} W_{r} \rightarrow V_{r^{\prime}} / \alpha_{r^{\prime}} W_{r^{\prime}}$ are clearly bijective, it follows from [B, I, (2.6) and (2.7)] that $\Phi$ is a covering, and hence defines an inclusion $\Gamma_{r} \leq \Gamma_{r^{\prime}}$.

This proves

(7.6) Proposition. Let $M$ be a group of order $m$. There are lattices $\Gamma_{r} \in$ Lat $_{u}\left(X_{2 m}\right) \quad(r \geq 1)$ such that for all $r, \Gamma_{r} \backslash X_{2 m}$ is a loop, $\left(\Gamma_{r}\right) \cong M^{r}$ for all $x \in X_{2 m}$, and $\operatorname{Vol}\left(\Gamma_{r} \backslash \backslash X_{2 m}\right)=1 / m^{r}$. If $r$ divides $r^{\prime}$. then $\Gamma_{r} \leq \Gamma_{r^{\prime}}$ with index $m^{r^{\prime}-r}$. If $m, r \geq 2$, then, $\Gamma_{r^{s}}(s=1,2,3, \ldots)$ is an infinite ascending chain in $\operatorname{Lat}_{u}\left(X_{2 m}\right)$.

(7.7) Example. Consider next the graph

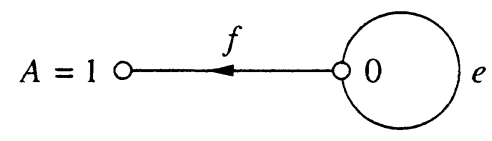

with indexing $i(e)=m=i(\bar{e}), i(f)=p, i(\bar{f})=q$ :

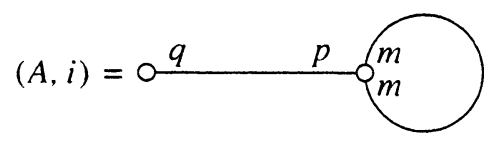

Choose groups $M, P, Q$ of orders $m, p, q$, respectively. For integers $r \geq 1$ define groupings $\mathbf{A}_{r}=\left(A, \mathscr{A}^{(r)}\right)$ of $(A, i)$ as follows: Let $V_{r}=M^{(\mathbf{Z} / r \mathbf{Z})}, \bar{W}_{r}$, and $\alpha_{r} \in \operatorname{Aut}\left(V_{r}\right)$ be as in (7.4).

$$
\begin{aligned}
& \mathscr{A}_{0}^{(r)}=V_{r} \times P, \\
& \mathscr{A}_{1}^{(r)}=V_{r} \times Q, \\
& \mathscr{A}_{e}^{(r)}=\mathscr{A}_{\bar{e}}^{(r)}=W_{r} \times P, \\
& \mathscr{A}_{f}^{(r)}=\mathscr{A}_{\bar{f}}^{(r)}=V_{r} .
\end{aligned}
$$


All edge monomorphisms are the obvious inclusions except for $\alpha_{e}=\alpha_{r} \times$ $\operatorname{Id}_{P}: W_{r} \times P \rightarrow V_{r} \times P$. The effectiveness of $\mathbf{A}_{r}$ follows easily from that of example (7.4) (cf. [B, I, (1.23)]). Put $X=(\widetilde{A, i}, 0)$ and $\Gamma_{r}=\pi_{1}\left(\mathbf{A}_{r}, 0\right) \in \operatorname{Lat}_{u}(X)$. Then $\Gamma_{r} \backslash X=A$ and

$$
\operatorname{Vol}\left(\Gamma_{r} \backslash \backslash X\right)=\frac{1}{p m^{r}}+\frac{1}{q m^{r}}=\frac{1}{m^{r}}\left(\frac{1}{p}+\frac{1}{q}\right)=\frac{p+q}{p q m^{r}} .
$$

If $r$ divides $r^{\prime}$, then, just as in (7.5), we obtain a covering morphism $\mathbf{A}_{r} \rightarrow$ $\mathbf{A}_{r^{\prime}}$ inducing an inclusion $\Gamma_{r} \leq \Gamma_{r^{\prime}}$

Note finally that if $q=2 m+p$, then $X=X_{q}$. This proves

(7.8) Proposition. Let $n=2 m+p$ with $m, p$ integers $\geq 1$. Then there exist $\Gamma_{r} \in \operatorname{Lat}_{u}\left(X_{n}\right) \quad(r \geq 1)$ such that

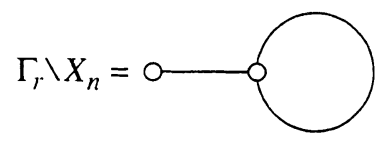

is independent of $r$, and

$$
\operatorname{Vol}\left(\Gamma_{r} \backslash X_{n}\right)=\frac{2(m+p)}{m^{r} \cdot p \cdot(2 m+p)} .
$$

If $r$ divides $r^{\prime}$, then $\Gamma_{r} \leq \Gamma_{r^{\prime}}$. If $m, r \geq 2$, then $\Gamma_{r^{s}}(s=1,2,3, \ldots)$ is an infinite ascending chain in Lat $_{u}\left(X_{n}\right)$.

Taking $p=1$ and $m \geq 2,(7.6)$ and (7.8) produce infinite ascending chains in Lat $_{u}\left(X_{n}\right)$ for all $n \geq 4$. For part (a) of Theorem (7.1) it remains to treat $X_{3}$, which we do as follows.

(7.9) Example. Let

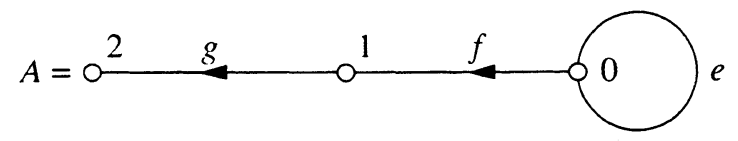

with indexing $i(e)=i(\bar{e})=i(f)=i(\bar{f})=1, i(g)=2$, and $i(\bar{g})=3$.

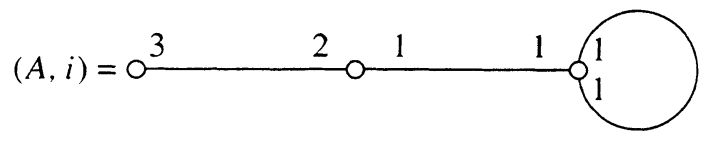


Then $(\widetilde{A, i}, 0)=X_{3}$. Let $M=\mathbf{Z} / 2 \mathbf{Z}$. For $r \geq 1$ define $V_{r}=M^{(\mathbf{Z} / r \mathbf{Z})}, W_{r}$, and $\alpha_{r}$ as in (7.4). Putting $Q=\mathbf{Z} / 3 \mathbf{Z}$, define

$$
A_{r}=\left(A, \mathscr{A}^{(r)}\right)=0 \frac{Q \times W_{r}}{W_{r}} \stackrel{0 \frac{V_{r}}{V_{r}}}{\circ V_{r}} V_{r}
$$

where all edge monomorphisms are the obvious inclusions except for $\alpha_{e}=\alpha_{r}$. Then just as in (7.4) we see that $\mathbf{A}_{r}$ is effective. Put

$$
\begin{aligned}
\Gamma_{r} & =\pi_{1}\left(\mathbf{A}_{r}, 0\right) \in \operatorname{Lat}_{u}\left(X_{3}\right) \\
& \cong\left\langle\left(Q \times W_{r}\right){ }_{W_{r}} V_{r}, \text { elexe } e^{-1}=\alpha_{r}(x) \text { for all } x \in V_{r}\right\rangle .
\end{aligned}
$$

Then $\Gamma_{r} \backslash X_{3}=A$ and

$$
\operatorname{Vol}\left(\Gamma_{r} \backslash X_{3}\right)=\frac{1}{2^{r}}+\frac{1}{2^{r}}+\frac{1}{3 \cdot 2^{r-1}}=\frac{1}{3 \cdot 2^{r-3}}
$$

If $r$ divides $r^{\prime}$, then, as in (7.5), we produce a covering $\mathbf{A}_{r} \rightarrow \mathbf{A}_{r^{\prime}}$ inducing an inclusion $\Gamma_{r} \leq \Gamma_{r^{\prime}}$.

(7.10) Proposition. There exist $\Gamma_{r} \in$ Lat $_{u}\left(X_{3}\right) \quad(r \geq 1)$ with the same quotient

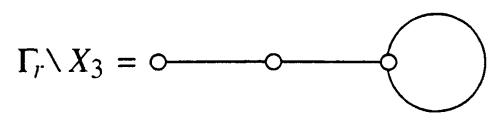

and

$$
\operatorname{Vol}\left(\Gamma_{r} \backslash \backslash X_{3}\right)=1 /\left(3 \cdot 2^{r-3}\right) \text {. }
$$

If $r$ divides $r^{\prime}$, then $\Gamma_{r} \leq \Gamma_{r^{\prime}}$. Hence, if $r \geq 2$, then $\Gamma_{r^{\prime}} \cdot(s=1,2,3, \ldots)$ is an infinite ascending chain in $\operatorname{Lat}_{u}\left(X_{3}\right)$.

(7.11) Lattices generated by finite groups. Consider a graph of groups $\mathbf{A}=$ $(A, \mathscr{A}), a_{0} \in A, X=\left(\widetilde{\mathbf{A}, a_{0}}\right)$, and $\Gamma=\pi_{1}\left(\mathbf{A}, a_{0}\right)$. There is a natural exact sequence

$$
1 \rightarrow N \rightarrow \Gamma \rightarrow \pi_{1}\left(A, a_{0}\right) \rightarrow 1,
$$

where $N$ is the subgroup of $\Gamma$ generated by all $\Gamma_{x}(x \in X)$ (cf. [B, I, (1.5), Example 3]). It follows that $\Gamma$ is generated by the stabilizers $\Gamma_{x}$ iff $A=\Gamma \backslash X$ is a tree. When $\Gamma$ is discrete, e.g., a lattice, this is equivalent to $\Gamma$ being generated by finite groups (cf. [B, II, (7.3)]).

Our construction of ascending chains of lattices made important use of a loop in the graph $A$; the automorphism $\alpha_{r}$ was decisive in establishing effectiveness. When, on the contrary, $A$ is a tree, such constructions are slightly more subtle.

We begin with an edge. 
(7.12) Amalgams. Let $A=0 \stackrel{e}{\longrightarrow} 01$ with indexing $i(e)=m_{0}, i(\bar{e})=m_{1}$; assume that $m_{i} \geq 2(i=0,1)$.

$$
A_{m_{0}, m_{1}}=(A, i)=\stackrel{m_{0} \quad m_{1}}{\stackrel{0}{\longrightarrow}}
$$

A grouping of $A_{m_{0}, m_{1}}$ is an "amalgam"

$$
\mathbf{A}=\left(\Gamma_{0} \stackrel{\alpha_{e}}{\leftarrow} \Gamma_{e} \stackrel{\alpha_{\bar{e}}}{\rightarrow} \Gamma_{1}\right)
$$

with $\left[\Gamma_{i}: \Gamma_{e}\right]=m_{i}(i=0,1)$, where we identify $\Gamma_{e}$ with its image in $\Gamma_{i}$ $(i=0,1)$. We have

and

$$
\Gamma=\pi_{1}(\mathbf{A}, 0) \cong \Gamma_{0} *_{\Gamma_{e}} \Gamma_{1}
$$

$$
X=(\widetilde{\mathbf{A}, 0})=X_{m_{0}, m_{1}},
$$

the bihomogeneous bipartite tree with indices $m_{0}, m_{1}$. A is effective, i.e., $\Gamma$ acts effectively on $X$, iff $\Gamma_{e}$ contains no subgroup $N \neq\{1\}$ which is normal in each $\Gamma_{i}$.

(7.13) Composite amalgams. Suppose that one of $m_{0}$ and $m_{1}$ is composite, say $m_{0}=p m$ with $p, m \geq 2$. Following an idea offered by Paul Fan (for which he referred to Djokovic [Dj]), we shall construct large families of lattices associated with $A_{m_{0}, m_{1}}$. Writing $q$ for $m_{1}$, choose groups $M, P, Q$ of respective orders $m, p, q$. Consider pairs $\left(S, s_{0}\right)$, where $S$ is a finite set with a transitive action of $P * Q$, and $s_{0} \in S^{Q}$ is a fixed point of $Q$; put $S^{\prime}=S-\left\{s_{0}\right\}$. Then we can form the wreath products $M^{S} \rtimes P$ and $M^{S^{\prime}} \rtimes Q$. With these we define the graph of groups

$$
\mathbf{A}_{\left(S, s_{0}\right)}=\frac{M^{S} \rtimes P \quad M^{s^{\prime}} \rtimes Q}{M^{S^{\prime}}},
$$

where the edge monomorphisms are the obvious inclusions. To show effectiveness note that

$$
\Gamma=\Gamma_{\left(S, s_{0}\right)}=\pi_{1}\left(\mathbf{A}_{\left(S, s_{0}\right)}, 0\right)=\left(M^{S} \rtimes P\right) *{ }_{M^{S^{\prime}}}\left(M^{S^{\prime}} \rtimes Q\right)
$$

has as quotient the wreath product $\Gamma^{\prime}=M^{S} \rtimes(P * Q)$ (obtained by making $Q$ centralize the $s_{0}$-factor of $\left.M^{S}\right)$. If $g \in P * Q$, then in $\Gamma^{\prime}, g M^{S^{\prime}} g^{-1}=$ $M^{g\left(S^{\prime}\right)}=\left\{x: S \rightarrow M \mid x\left(g\left(s_{0}\right)\right)=1\right\}$. Since $P * Q$ acts transitively on $S$, it follows that $\bigcap_{g \in P * Q} g M^{S^{\prime}} g^{-1}=\{1\}$ in $\Gamma^{\prime}$. Now suppose that $N \leq M^{S^{\prime}} \leq \Gamma$ is normalized by $M^{S} \rtimes P$ and $M^{S^{\prime}} \rtimes Q$, hence normal in $\Gamma$. Projecting to $\Gamma^{\prime}$ we see from the calculation above that $N=\{1\}$. Thus, $A_{\left(S, s_{0}\right)}$ is effective. We have $\Gamma_{\left(S, s_{0}\right)} \backslash X=œ$ and, with $s=|S|$,

$$
\operatorname{Vol}\left(\Gamma_{\left(S, s_{0}\right)} \backslash \backslash X\right)=\frac{1}{m^{s} p}+\frac{1}{m^{s-1} q}=\frac{1}{m^{s-1}}\left(\frac{1}{m p}+\frac{1}{q}\right)=\frac{1}{m^{s-1}}\left(\frac{1}{m_{0}}+\frac{1}{m_{1}}\right)
$$


Suppose that $T$ is another transitive $(P * Q)$-set, that $t_{0} \in T^{Q}$, and that $p: T \rightarrow S$ is a $(P * Q)$-equivariant map with $p\left(t_{0}\right)=s_{0}$. Then we obtain an embedding $M^{S} \rightarrow M^{T}$, inducing $M^{S^{\prime}} \rightarrow M^{T^{\prime}}$, and which is $(P * Q)$ equivariant. Hence, we obtain a morphism $\mathbf{A}_{\left(S, s_{0}\right)} \rightarrow \mathbf{A}_{\left(T, t_{0}\right)}$ which is easily seen to be a covering, and so we have an inclusion $\Gamma_{\left(S, s_{0}\right)} \leq \Gamma_{\left(T, t_{0}\right)}$.

It remains only to produce such pairs $\left(S, s_{0}\right)$ as above. These correspond bijectively to finite index subgroups $H_{S}$ in $G=P * Q$ such that $Q \leq H_{S}$. We then have $S=G / H_{S}$ and $s_{0}=1 \cdot H_{S} \in S$. The situation $\left(T, t_{0}\right) \rightarrow\left(S, s_{0}\right)$ above corresponds to an inclusion $H_{T} \leq H_{S}$. Since the group $G$ is infinite and residually finite, there exist $\left(S, s_{0}\right)$ with $s=|S|$ arbitrarily large. In fact, the intersection of all such $H_{S}$ is just $Q$. This proves

(7.14) Proposition. Let $m, p, q$ be integers $\geq 2$ and $X=X_{m p, q}$. Among $\Gamma \in \operatorname{Lat}_{u}(X)$ with a common edge-indexed quotient $I(\Gamma \backslash \backslash X) \stackrel{m p}{\stackrel{q}{q}} \circ$ and $\operatorname{Vol}(\Gamma \backslash \backslash X)=\frac{1}{m^{r}}(1 / m p+1 / q)$ for some $r>0$ (depending on $\Gamma$ ), there exist infinite ascending chains.

We can also use the construction of (7.13) to prove

(7.15) Proposition. Let $q=m+1$ with $m$ an integer $\geq 2$. Among $\Gamma \in$ Lat $_{u}\left(X_{q}\right)$ with a common edge-indexed quotient $I\left(\Gamma \backslash \backslash X_{q}\right)=0 \stackrel{0 \quad 1}{0} \stackrel{m \quad q}{\circ} 0$ and $\operatorname{Vol}\left(\Gamma \backslash \backslash X_{q}\right)=2 / m^{s}$ for some $s>0$, there exist infinite ascending chains.

Proof. Choose groups $M, Q$ of respective orders $m, q$, and put $P=Q$. As in (7.13), consider pairs $\left(S, s_{0}\right)$ with $S$ a transitive $(P * Q)$-set and $s_{0} \in S^{Q}$, and put $S^{\prime}=S-\left\{s_{0}\right\}$. Then define

$$
\mathbf{A}_{\left(S, s_{0}\right)}=\frac{M^{S} \times P}{M^{S}} \underset{\sim}{\circ} \frac{M^{S} \quad M^{S^{\prime}} \times Q_{0}}{M^{S^{\prime}}},
$$

where the edge monomorphisms are the obvious inclusions. This is a grouping of

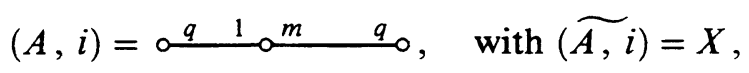

and it follows easily from the discussion in (7.13) that it is effective. Put $\Gamma_{\left(S, s_{0}\right)}=\pi_{1}\left(\mathbf{A}_{\left(S, s_{0}\right)}, *\right)$. If $\left(T, t_{0}\right) \rightarrow\left(S, s_{0}\right)$ is a $(P * Q)$-morphism of such pairs, then we obtain inclusions $M^{S} \rtimes P \rightarrow M^{T} \rtimes P$, etc., whence a covering morphism, hence an inclusion $\Gamma_{\left(S, s_{0}\right)} \leq \Gamma_{\left(T, t_{0}\right)}$. We have $I\left(\Gamma_{\left(S, s_{0}\right)} \backslash \backslash X_{q}\right)=(A, i)$ and, putting $s=|S|$,

$$
\begin{aligned}
\left.\operatorname{Vol}\left(\Gamma_{\left(s, s_{0}\right)}\right) \backslash X_{q}\right) & =\frac{1}{m^{s} q}+\frac{1}{m^{s}}+\frac{1}{m^{s-1} q} \\
& =\frac{1}{m^{s} q}(1+q+m)=\frac{2 q}{m^{s} q}=\frac{2}{m^{s}} .
\end{aligned}
$$

Now Proposition (7.15) follows, as did (7.14), from the observations at the end of (7.13). 
(7.16) Prime amalgams. Consider the edge-indexed graph

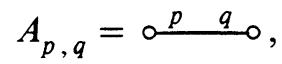

where we now assume that $p$ and $q$ are primes. The covering tree is $X_{p, q}=$ $\tilde{A}_{p, q}$. Finite effective groupings of $A_{p, q}$ correspond to lattices $\Gamma \in \operatorname{Lat}_{u}\left(X_{p, q}\right)$ which are transitive on geometric edges, but not on vertices.

Conjecture. There are (up to isomorphism) only finitely many effective finite groupings of $A_{p, q}$.

Equivalently, there are only finitely many conjugacy classes of $\Gamma \in \operatorname{Lat}_{u}\left(X_{p, q}\right)$ which are transitive on geometric edges, but not on vertices.

Let $\mathbf{A}=\left(\begin{array}{ll}\Gamma_{0} & \Gamma_{1} \\ \Gamma_{c}\end{array}\right)$ be an effective finite grouping of $A_{p, q}:\left[\Gamma_{0}\right.$ : $\left.\Gamma_{e}\right]=p,\left[\Gamma_{1}: \Gamma_{e}\right]=q$. The corresponding lattice is $\Gamma=\Gamma_{0} *_{\Gamma_{e}} \Gamma_{1}$. The conjecture above has been proved by $P$. Fan $[F]$ under the additional assumption that $\Gamma_{e}$ is an $l$-group for some prime $l$ different from $p$ and $q$. When $p=$ $q=3, \Gamma_{e}$ is automatically a 2-group, and this case was first treated in a wellknown paper of Goldschmidt [Go]. The case $p=q=2$ is trivial: $X_{2,2}=X_{2}$ is the linear tree and the unique effective $(2,2)$-amalgam is the infinite dihedral group $(\mathbf{Z} / 2 \mathbf{Z}) *(\mathbf{Z} / 2 \mathbf{Z})$. More generally, consider:

(7.17). The case $A_{p, 2}=\circ \frac{p 2}{\Gamma} \circ$ with $p>2$. Then $X_{p, 2}$ is the barycentric subdivision of $X_{p}$. Let $\Gamma \in \operatorname{Lat}_{u}\left(X_{p, 2}\right)$ with $\Gamma \backslash X_{p, 2}=\circ-\circ$. Then $\Gamma$ defines (by suppressing vertices of index 2), a lattice $\Gamma^{\prime} \in \operatorname{Lat}_{u}\left(X_{p}\right)$ that is transitive on both vertices and oriented edges; hence $\Gamma^{\prime}$ contains an inversion. According to [B, II, (6.3)], $\Gamma^{\prime}$ contains an index 2 subgroup $\Gamma^{0}$ without inversions and even length function. Consider $(A, i)=I\left(\Gamma^{0} \backslash \backslash X_{p}\right)$. The group $\Gamma^{\prime} / \Gamma^{0}=\{1, \sigma\}$ acts on $(A, i)$, transitively on vertices and on oriented edges, preserving indices. If follows that $A$ has only one geometric edge, and so

$$
(A, i)=\circ \frac{p \quad p}{2} \text {. }
$$

Conclusion. Let $\Gamma \in \operatorname{Lat}_{u}\left(X_{p, 2}\right)$ with $I\left(\Gamma \backslash \backslash X_{p, 2}\right)=\circ \frac{p \quad 2}{\circ}$. If $p$ is odd, then there is a $\Gamma^{0} \in \operatorname{Lat}_{u}\left(X_{p}\right)$ with $I\left(\Gamma^{0} \backslash \backslash X_{p}\right)=\circ \underline{p \quad p} \circ$ and $\Gamma^{0}$ is isomorphic to a subgroup of index 2 in $\Gamma$.

In this way, for example, (3.2)-amalgams can be described in terms of $(3,3)$ amalgams, covered by Goldschmidt's work.

(7.18) Example. Let $p$ be a prime. The following construction provides lattices $\Gamma_{e} \in \operatorname{Lat}_{u}\left(X_{p+1}\right)$ such that

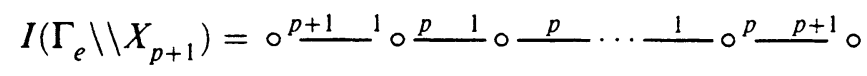


with $e+2$ vertices, and with volume

$$
v_{e}=\operatorname{Vol}\left(\Gamma_{e} \backslash \backslash X_{p+1}\right)=\frac{1}{p^{r(e)}}\left(\frac{p^{e}+1}{p+1}+r(e)\right),
$$

where $r(e)=\left(p^{e}-1\right) /(p-1)$. Thus, $v_{e} \rightarrow 0$ as $e \rightarrow \infty$. Explicitly, $\Gamma_{e}=$ $\pi_{1}\left(\mathbf{A}_{e}, *\right)$, where

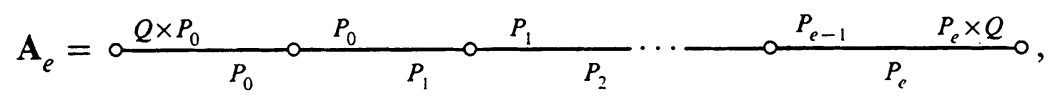

and where $Q$ is any group of order $p+1, P=P_{0}$ is a Sylow $p$-subgroup of the symmetric group $S_{p^{e}}, P_{i+1}$ has index $p$ in $P_{i}$, and $P_{e}$ is a point stabilizer in $P$ (acting on $\left.\left\{1,2, \ldots, p^{e}\right\}\right)$. Then $|P|=p^{r(e)}$, and $P_{e}$ contains no normal subgroup $\neq 1$ of $P$, so $\mathbf{A}_{e}$ is effective.

(7.19) Example. The following constructions provide many uniform lattices on the same tree with the same covolume, thus establishing part (b) of Theorem (7.1).

Let $M$ be a group of order $|M|=m \geq 2$. For $r \geq 1$, write $M^{r}$ for $M^{(\mathbf{Z} / r \mathbf{Z})}$ and $\alpha_{r}$ for the automorphism $\alpha_{r}(x)(i)=x(i+1)$ for $x \in M^{r}$. Let

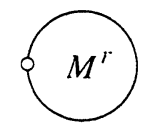

abbreviate the loop of groups

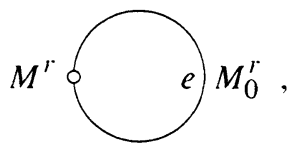

where $M_{0}^{r}=\left\{x \in M^{r} \mid x(0)=1\right\}$, and for $x \in M_{0}^{r}, \alpha_{\bar{e}}(x)=x$ and $\alpha_{e}(x)=$ $\alpha_{r}(x)$. We have seen (Example (7.4)) that this is an effective finite grouping of

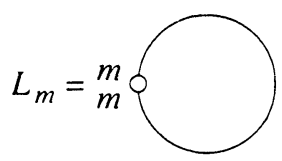


Let $N$ be an integer $>0$, and consider the following edge-indexed graph $\left(A_{N}, i\right)$, where unlabeled edges are understood to have index 1:

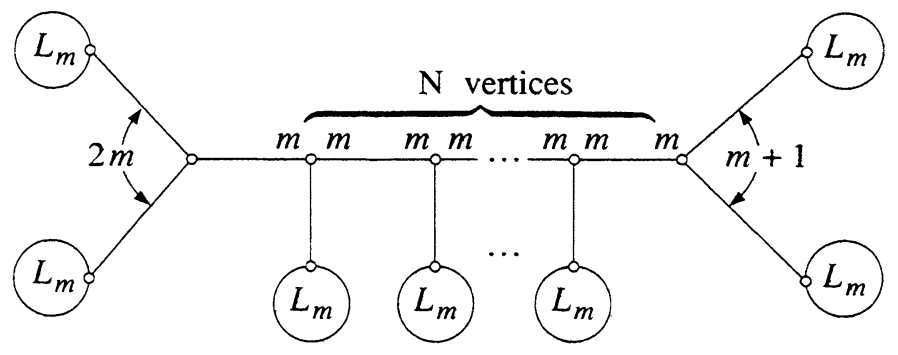

The notation

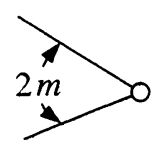

on the left signifies $2 m$ copies of

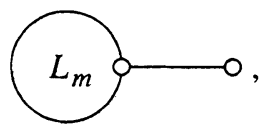

all issuing from the same right-hand vertex, and similarly on the right with

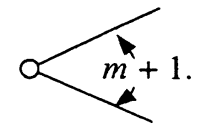

Note that $\left(\widetilde{A_{N}}, i\right)=X_{2 m+1}, A_{N}$ has $2 m+1+2 N+m+2=2 N+3(m+1)$ vertices. Now we define a finite grouping $\mathbf{A}_{r}=\left(A, \mathscr{A}^{(r)}\right)$ of $\left(A_{N}, i\right)$ as follows, using the abbreviation

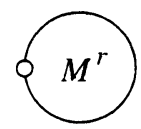

introduced above.

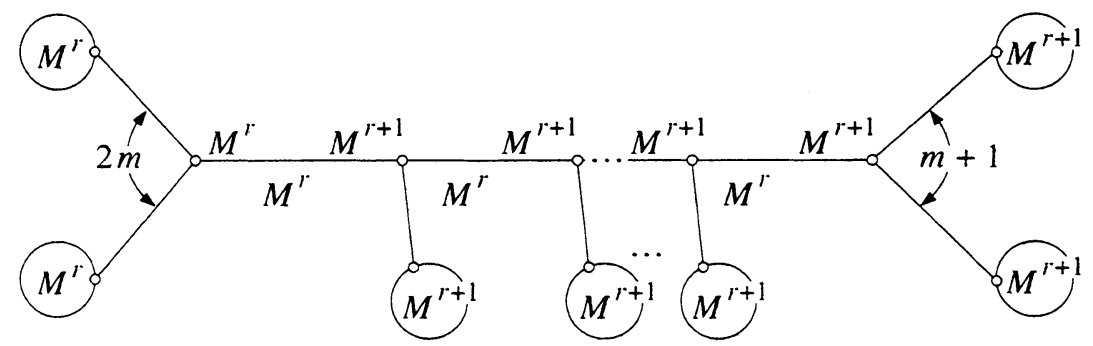


Here the edge monomorphisms outside the loops are either identities or the obvious isomorphism $M^{r} \rightarrow M_{0}^{r+1} \subset M^{r+1}$. Since

\section{$M^{r}$}

is effective, so also is $\mathbf{A}_{r}$ (cf. [B; I, (1.24)]). Let $\Gamma_{r}=\pi_{1}\left(\mathbf{A}_{r}\right) \in \operatorname{Lat}_{u}\left(X_{2 m+1}\right)$. Then the covolume is

$$
\begin{aligned}
v_{r} & =\operatorname{Vol}\left(\Gamma_{r} \backslash X_{2 m+1}\right)=\frac{2 m+1}{m^{r}}+\frac{2 N}{m^{r+1}}+\frac{m+2}{m^{r+1}} \\
& =\frac{1}{m^{r+1}}\left(2 m^{2}+m+2 N+m+2\right) \\
& =\frac{2}{m^{r+1}}\left(m^{2}+m+1+N\right),
\end{aligned}
$$

so

$$
N=\frac{v_{r} \cdot m^{r+1}}{2}-\left(m^{2}+m+1\right)
$$

Fix an even integer $v=2 v_{0}>0$, and define

$$
N_{r}=v_{0} \cdot m^{r+1}-\left(m^{2}+m+1\right)
$$

For $r \geq 2, N_{r}>0$. Taking $N=N_{r}$ above we get $v_{r}=v$ for all $r \geq 2$.

Conclusion. Let $m$ be an integer $\geq 2$, and $v$ an even integer $>0$. There exist lattices $\Gamma_{r} \in \operatorname{Lat}_{u}\left(X_{2 m+1}\right) \quad(r \geq 2)$ such that $\operatorname{Vol}\left(\Gamma_{r} \backslash \backslash X_{2 m+1}\right)=v$ but $\Gamma_{r}$ and $\Gamma_{r^{\prime}}$ are nonconjugate for $r \neq r^{\prime}$; in fact, $\Gamma_{r} \backslash X_{2 m+1}$ has $v m^{r+1}-2 m^{2}+m+1$ vertices. 
(7.20) Example. To handle $X_{n}$ for $n$ even consider the following edge-indexed graph $\left(A_{N}, i\right)$ :

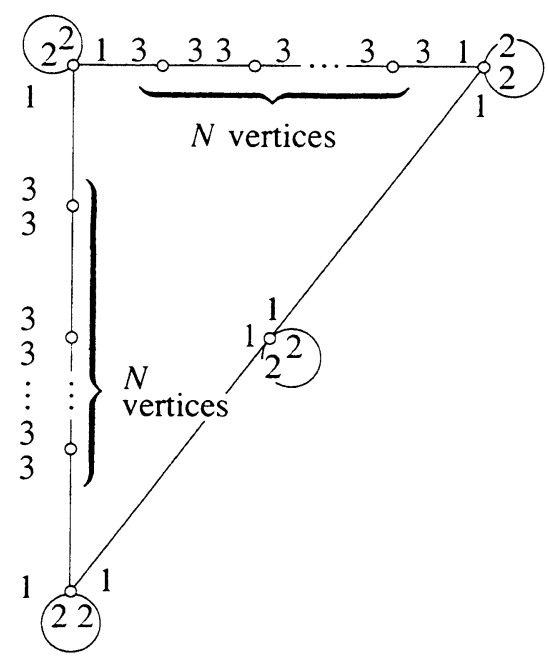

Note that $\left(\widetilde{A_{N}}, i\right)=X_{6}$ and $A_{N}$ has $2 N+4$ vertices. Let $M=\mathbf{Z} / 2 \mathbf{Z}$, $T=\mathbf{Z} / 3 \mathbf{Z}$, and consider the following grouping $\mathbf{A}_{r}$ of $\left(A_{N}, i\right)$, where we use the abbreviation $M^{r}$ of $(7.19)$ above.

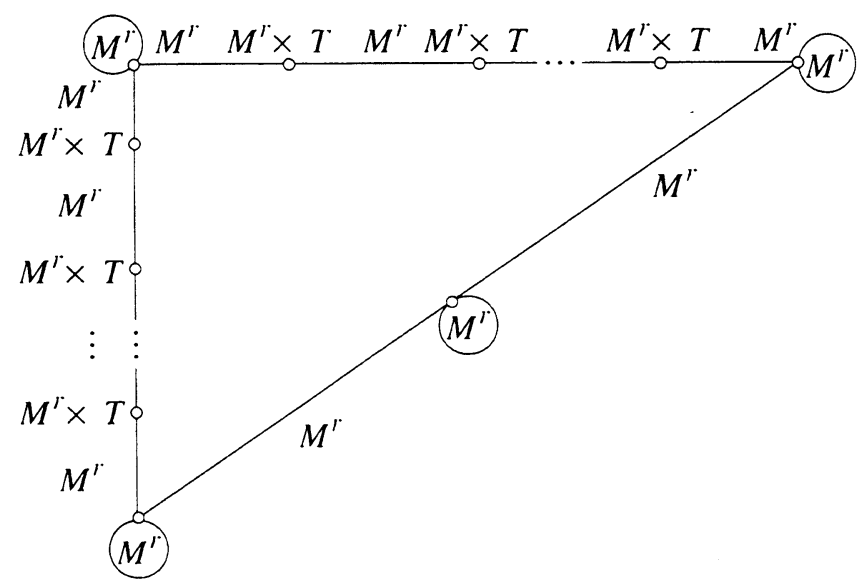

Put $\Gamma_{r}=\pi_{1}(\mathbf{A}) \in \operatorname{Lat}_{u}\left(X_{6}\right)$. Then the covolume is

$$
v_{r}=\operatorname{Vol}\left(\Gamma_{r} \backslash \backslash X_{6}\right)=\frac{4}{2^{r}}+\frac{2 N}{3 \cdot 2^{r}}=\frac{6+N}{3 \cdot 2^{r-1}},
$$

so $N=3 \cdot 2^{r-1} \cdot v_{r}-6$. Let $v$ be any integer $>0$, and put $N_{r}=3 \cdot 2^{r-1} \cdot v-6$. 
Then $N_{r}>0$ for $r \geq 2$, and even for $r=1$ if $v>1$. With $N=N_{r}$ we then see that $v_{r}=v$, independently of $r$.

Now add new edges to $\left(A_{N}, i\right)$ to obtain $\left(B_{N}, j\right)$ as follows:

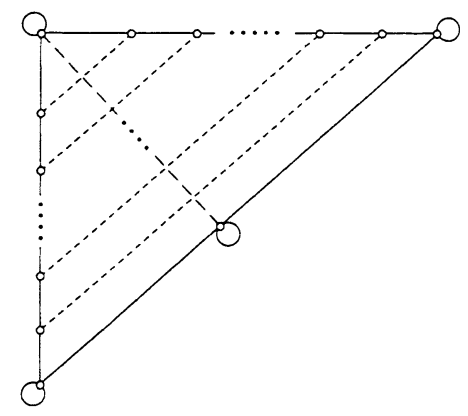

Here, for a chosen integer $t \geq 0$, each broken edge denotes $t$ geometric edges, each with index 1 for both orientations. In $\mathbf{A}_{r}$, the groups at the ends of each of these new edges are identical, so we can put the same group on the new edge to extend $\mathbf{A}_{r}$ to a grouping $\mathbf{B}_{r}$ of $\left(B_{N}, j\right)$ with the same vertex groups, and hence the same volume. Note that $\left(B_{N}, j\right)=X_{6+t}$. Putting $\Gamma_{t, r}=\pi_{1}\left(\mathbf{B}_{r}\right) \in$ $\operatorname{Lat}_{u}\left(X_{6+t}\right)$ we have $\operatorname{Vol}\left(\Gamma_{t, r} \backslash \backslash X_{6+t}\right)=\operatorname{Vol}\left(\Gamma_{r} \backslash \backslash X_{6}\right)=v_{r}$. Choosing $N=N_{r}$ as above we make these volumes equal $v$, independently of $r$.

Conclusion. For $n \geq 6$ and $v$ any integer $>0$, there exist lattices $\Gamma_{r} \in$ $\operatorname{Lat}_{u}\left(X_{n}\right) \quad(r \geq 2)$ such that $\operatorname{Vol}\left(\Gamma_{r} \backslash \backslash X_{n}\right)=v$ and $\Gamma_{r} \backslash X_{n}$ has $3 \cdot 2^{r} \cdot v-8$ vertices. Hence the $\Gamma_{r}$ are pairwise nonconjugate.

\section{ACKNOWLEDGMENTS}

Alex Lubotzky first proposed many of the questions addressed here, and he was a constant source of stimulation. Robert Edwards first brought Leighton's paper [L] to our attention. J.-P. Serre and Walter Neumann furnished helpful observations on commensurability groups. For background information and constructions of finite effective groupings we received valuable assistance from Robert Guralnick and Paul Fan, and helpful references from Michael Aschbacher. Geoffrey Mess suggested Lemma (B.12). Armand Borel proposed the question answered by $(7.1)(\mathrm{b})$; his paper [Bo] was very helpful to us. We have had helpful conversations with and encouragement from Roger Alperin, Peter Sarnak, Isaac Effrat, and J. Tits. We are grateful to all of the above. Finally, we thank Ying-Sheng Liu for a careful reading of the manuscript and several important corrections. 


\section{REFERENCES}

[AB] R. Alperin and H. Bass, Length functions of group actions on $\wedge$-trees, in Combinatorial Group Theory and Topology, Ann. of Math. Stud., no. 111, Princeton Univ. Press, Princeton, NJ, 1987, pp. 265-378.

[B] H. Bass, Covering theory for graphs of groups, J. Pure Appl. Algebra, submitted.

[B2] _- Group actions on non-archimedean trees, Proc. MSRI Conf. on Arboreal Group Theory, 1988.

[BMS] H. Bass, J. Milnor, and J.-P. Serre, Solution of the congruence subgroup problem for $S L_{n}$ $(n \geq 3)$ and $S p_{2 n}(n \geq 2)$, Inst. Hautes Études Sci. Publ. Math. 33 (1967), 59-137.

[Bo] A. Borel, Commensurability classes and volumes of hyperbolic 3-manifolds, Ann. Scuola Norm. Sup. Pisa Cl. Sci. (4) 8 (1981), 1-33.

[BT] F. Bruhat and J. Tits, Groupes algébriques simples sur un corps local, Proc. Conf. on Local Fields (T. A. Springer, ed.), Springer-Verlag, Driebergen, 1967, pp. 23-36.

[D] J. Dieudonné, Treatise on analysis, vol. II, Academic Press, New York, 1976.

[F] P. Fan, Amalgams of prime index, J. Algebra 98 (1986), 375-421.

[Dj] D. Djokovic, A class of finite group amalgams, Proc. Amer. Math. Soc. 80 (1980), 22-26.

[Go] D. M. Goldschmidt, Automorphisms of trivalent graphs, Ann. of Math. (2) 111 (1980), 377-406.

[Im] W. Imrich, Subgroup theorems and graphs, Combinatorial Mathematics V, Lecture Notes in Math., vol. 622, Springer-Verlag, Berlin and New York, 1977.

[K] R. Kulkarni, Lattices on trees, automorphisms of graphs, free groups, surfaces, preprint, CUNY, 1988.

[L] F. T. Leighton, Finite common coverings of graphs, J. Combin. Theory Ser. B 33 (1982), 231-238.

[Lub] A. Lubotzky, Trees and discrete subgroups of Lie groups over local fields, Bull. Amer. Math. Soc. (N.S.) 20 (1988), 27-31.

[Rag] M. S. Raghunathan, Discrete subgroups of Lie groups, Ergebnisse der Math., vol. 68, Springer-Verlag, 1972.

[S] J.-P. Serre, Trees, Springer-Verlag, New York, 1980.

[S2] Cohomologie des groupes discrets, Ann. of Math. Stud., no. 70, Princeton Univ. Press, Princeton, NJ, 1971, pp. 71-169.

[St] J. Stallings, Topology of finite graphs, Invent. Math. 71 (1983), 551-565.

Department of Mathematics, Columbia University, New York, New York, 10027

Department of Mathematics, City University of New York Graduate Center, New YORK, NEW YORK 10036 